\title{
INSTRUMENTATION AND BEAM DYNAMICS STUDY OF ADVANCED ELECTRON-PHOTON FACILITY IN INDIANA UNIVERSITY
}

\author{
Tianhuan Luo
}

Submitted to the faculty of the University Graduate School

in partial fulfillment of the requirement

for the degree

Doctor of Philosophy

in the Department of Physics,

Indiana University

August, 2011 
Accepted by the Graduate Faculty, Indiana Univeristy, in partial fulfillment of the requirement for the degree of Doctor of Philosophy.

Shyh-Yuan Lee, Ph.D.

John P. Carini, Ph.D.

Doctoral

Committee

Joshua C. Long, Ph.D.

August 8th, 2011

Paul E. Sokol, Ph.D. 
Copyright @ 2011 by

Tianhuan Luo

ALL RIGHTS RESERVED 
To my parents 


\section{Acknowledgments}

First and foremost I want to thank my advisor Prof. Shyh-Yuan Lee, who has given me tremendous help over the past 3 years. I appreciate his contributions of time, ideas and funding to make my Ph.D. experience productive and stimulating. What I benefited is not only from his broad knowledge and deep understanding of accelerator physics but also from his hardworking attitude, passion for science and unrelenting creativity. The inspiration from him will last for a long time in my life.

Many thanks go to all my teachers in the Indiana University Physics Department and United States Particle Accelerator School, especially Prof. Brian Serot and Prof. Herbert Fertig in Indiana University, Dr. Bill Ng in Fermi National Lab and Dr. Fernando Sannibale in Lawrence Berkeley National Lab. Thanks for all the classes, the discussions and the encouragements.

I want to thank my group members Xiaoying Pang, Yichao Jing, Xin Wang, Honghuan Liu and Alfonse Pham for all the discussions, collaboration and the ultra friendly atmosphere in our group.

I owe a lot to our ALPHA group members, Gary East, Patrick McChesney, Tom Rinckle, Chandra Romel, Jack Doskow, Max Ellis and everyone else. Great appreciation goes to Prof. Paul Sokol for his inspiring suggestions and full support on my work. I want to thank Prof. John Carini and Doc MacDonald for the great help on my RF work. My fast extraction kicker design could not be finished without the help from Dr. Derun Li, Dr. Stefano De Santis and Dr. John Byrd in Lawrence Berkeley National Lab. I also appreciate their hospitality when I visited Berkeley.

I am grateful to everyone in my thesis committee for their patient reading and very helpful suggestions on my thesis. Especially I appreciate the tremendous help from Prof. Josh Long and Prof Don Summers on my thesis writing. 
I want to thank my dear friends, Lihuei, Liming, Xiaoying, Yiting, Xin, Zhaowen, Yu, Peng, Xiling, Juan, Meng, Weiwei, Benben, and many others I cannot name one by one here, for all the joyful memories and brother-sister hood we shared with each other. And many thanks go to Maciej for his great companionship.

Finally, I want to thank my family, especially my parents, for all the education, support, freedom and unconditional love. 


\section{Abstract}

The Advanced eLectron-PHoton fAcility (ALPHA) is a compact electron accelerator under construction and being commissioned at the Indiana University Center for Exploration of Energy and Matter (CEEM). In this thesis, we have studied the refurbished Cooler Injector Synchrotron (CIS) RF cavity using both the transmission line model and SUPERFISH simulation. Both low power and high power RF measurements have been carried out to characterize the cavity. Considering the performance limit of ferrite, we have designed a new ferrite loaded, co-axial quarter wave like cavity with similar structure but a more suitable ferrite material. We have also designed a traveling wave stripline kicker for fast extraction by POISSON and Microwave Studio. The strips' geometry is trimmed to maximize the uniformity of the kicking field and match the impedance of the power cables. The time response simulation shows the kicker is fast enough for machine operation. The pulsed power supply requirement has also been specified. For the beam diagnosis in the longitudinal direction, we use a wideband Wall Gap Monitor (WGM) served in CIS. With proper shielding and amplification to get good WGM signal, we have characterized the injected and extracted beam signal in single pass commissioning, and also verified the debunching effect of the ALPHA storage ring. A modulation-demodulation signal processing method is developed to measure the current and longitudinal profile of injected beam. By scanning the dipole strength in the injection line, we have reconstructed the tomography of the longitudinal phase space of the LINAC beam. In the accumulation mode, ALPHA will be operated under a low energy and high current condition, where intra beam scattering (IBS) becomes a dominant effect on the beam emittance. A self consistent simulation, including IBS effect, gas scattering and linear coupling, has been carried out to calculate the emittance of the stored beam. 


\section{Contents}

Acceptance

Acknowledgments $\quad$ v

Abstract vii

1 Introduction 1

2 Introduction to ALPHA $\quad 4$

2.1 ALPHA storage ring design concept . . . . . . . . . . . 4

2.2 Radiation effect testing . . . . . . . . . . . . . . . 6

2.3 ALPHA beam physics study . . . . . . . . . . . . . 8

2.3.1 Intrabeam Scattering . . . . . . . . . . . . 8

2.3.2 Touschek Life Time . . . . . . . . . . . . . . . . . . . . 9

2.3.3 Beam manipulation by Gradient Damping Wiggler . . . . . . 9

3 The RF Cavity 10

3.1 Introduction of RF Cavity . . . . . . . . . . . . . . . . 10

3.2 Touschek Effect . . . . . . . . . . . . . . . . . . . . 13

3.3 Refurbishing of CIS RF Cavity . . . . . . . . . . . . . 14

3.3.1 The CIS RF Cavity . . . . . . . . . . . . . . . . 15 
3.3.2 The Frequency Tuning and impedance matching of CIS RF Cavity 16

3.3.3 The Quality Factor Q measurement . . . . . . . . . . . . . 18

3.3.4 Internal Capacitance Measurement . . . . . . . . . . . . . . . 21

3.3.5 The Shunt Impedance Measurement by Bead Pull ..... 24

3.4 Transmission Line Analysis of Ferrite-Loaded Cavity . . . . . . . . . 26

3.4.1 The Ferrite in the co-axial cavity. . . . . . . . . . . . 26

3.4.2 The Co-axial Resonators . . . . . . . . . . . . . . 29

3.4.3 Transmission line Analysis on CIS Cavity . . . . . . . . . . . . 32

3.5 High Power Test of shortened CIS RF Cavity . . . . . . . . . . . 37

3.5.1 Shortened CIS RF Cavity . . . . . . . . . . . . . . 37

3.5.2 High Power Test . . . . . . . . . . . . . . . . 39

3.6 The Performance Limit of CIS RF Cavity . . . . . . . . . . . . . . . 40

3.6.1 The High Loss Effect . . . . . . . . . . . . . . . . . . . . . 41

3.6.2 The limit of the ferrite of CIS RF Cavity . . . . . . . . . . 41

3.6.3 A more suitable ferrite material . . . . . . . . . . . . . . . 42

3.7 Design of the New RF Cavity . . . . . . . . . . . . . . . 44

3.7.1 The SUPERFISH Design of the New RF Cavity . . . . . . . . 44

4 Stripline Fast Extraction Kicker $\quad 50$

4.1 Specification of the Kicker . . . . . . . . . . . . . . 50

4.2 Numerical Simulation . . . . . . . . . . . . . . . . 52

4.2.1 2D Poisson Simulation . . . . . . . . . . . . . 53

4.2.2 3D Microwave Studio Simulation . . . . . . . . . . . 54

4.3 Requirement of Power Supply . . . . . . . . . . . . . . . . . 61

4 Longitudinal Beam Characterization $\quad 63$

4.1 The wide band Wall Gap Monitor . . . . . . . . . . . . . . . 64 
4.2 Bench test of WGM . . . . . . . . . . . . . . . . . 67

4.2.1 Effective impedance $R$. . . . . . . . . . . . . . . . 68

4.2.2 Bandwidth....................... 70

4.3 Measurement of the injected beam . . . . . . . . . . . . . . . . . 72

4.3.1 Signal shielding and amplification . . . . . . . . . . . 72

4.3.2 Signal processing with modulation-demodulation scheme . . . 73

4.3.3 Charge and longitudinal profile of the injected beam . . . . . 81

4.4 Longitudinal tomography of the LINAC beam . . . . . . . . . . . 82

4.4.1 G4beamline simulation of the injected beam . . . . . . . . 82

4.4.2 Longitudinal tomography of the injected beam . . . . . . . . . 87

4.5 Extraction beam line measurement . . . . . . . . . . . . . . . 88

5 Characterization of stored beam in ALPHA ring $\quad 91$

5.1 Emittance of an electron storage ring . . . . . . . . . . . . . . 91

5.1.1 Synchrotron radiation ................ 93

5.1 .2 Quantum fluctuation ................. 93

5.1.3 Gas scattering ..................... 94

5.1.4 Intrabeam Scattering . . . . . . . . . . . . . . 96

5.2 Equilibrium Emittance Calculation of ALPHA Storage Ring . . . . . 97

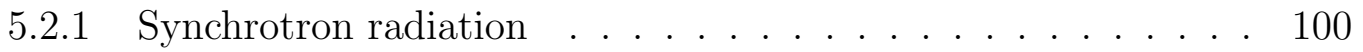

5.2.2 Quantum fluctuation ................ 100

5.2 .3 Gas scattering . . . . . . . . . . . . . 101

5.2 .4 Intrabeam Scattering . . . . . . . . . . . . . . . 101

5.2.5 Transverse Linear Coupling . . . . . . . . . . . . . . 101

5.2.6 The calculation procedure . . . . . . . . . . . . . . 102

5.3 Estimation Results and discussion . . . . . . . . . . . . . . 103 


\section{List of Tables}

3.1 Parameters of M4C21A ferrite torus . . . . . . . . . . . . . . . 44

4.1 Kicker Specification. . . . . . . . . . . . . . . 51

5.1 ALPAH Storage Ring Parameters . . . . . . . . . . . . . . . 97 


\section{List of Figures}

2.1 Simplified layout of ALPHA . . . . . . . . . . . . . 5

2.2 Beam debunching illustration . . . . . . . . . . . . . . . 7

3.1 The Structure of the CIS cavity . . . . . . . . . . . . . . 15

3.2 Bias Current versus resonant frequency . . . . . . . . . . . . . . 17

3.3 Resonant frequency drift with time . . . . . . . . . . . . 18

3.4 Q circle for linear fit . . . . . . . . . . . . . . . . . 20

3.5 Linear-fit for $Q_{L} \quad \ldots \ldots \ldots \ldots$. . . . . . . . . . . . 21

3.6 The resonant frequency tuning by external capacitor . . . . . . . . . 22

3.7 The linear fitting of $N-\frac{1}{f_{0}{ }^{2}} \ldots \ldots \ldots \ldots . \ldots \ldots$

3.8 Bead-pull measurement set up, drawn by Jack Doskow . . . . . . . . 24

3.9 Shunt Impedance Measurement of CIS RF Cavity by high power test. 27

3.10 Parallel resonant circuit with ferrite-cored inductor . . . . . . . . . . 28

3.11 Equivalent circuit of co-axial resonator. . . . . . . . . . . . . . 30

3.12 Typical resonator structure . . . . . . . . . . . . . . . . . 31

3.13 Upper half cavity structure in SUPERFISH simulation. . . . . . . . . 33

3.14 Frequency tuning by ferrite permeability . . . . . . . . . . . . 35

3.15 The effective length calculation. . . . . . . . . . . . . 36

3.16 The SUPERFISH simulation of the shortened CIS RF cavity . . . . . 38 
3.17 The High Power Test: $V_{R F} \ldots \ldots \ldots \ldots$

3.18 THe High Power Test: $R_{s h} \ldots \ldots \ldots \ldots \ldots \ldots$

3.19 The Example of High Loss Effect . . . . . . . . . . . . . 42

3.20 The High Loss Effect threshold measurement of Philips 8C12 . . . . 43

3.21 The SUPERFISH design of the new ferrite cavity $\ldots \ldots \ldots$

3.22 The magnetic field distribution of 1 st harmonic mode. . . . . . . . . 45

3.23 The resonant frequency scan by SUPERFISH $\ldots \ldots \ldots \ldots$

3.24 Magnetic field distribution of 2nd harmonic mode. . . . . . . . . 47

3.25 Magnetic field distribution of 3rd harmonic mode. . . . . . . . . 47

3.26 The HLE Threshold Measurement of M4C12A . . . . . . . . . . 49

4.1 Illustration of Kicker structure and kicking field . . . . . . . . 52

4.2 2D electric field line in a quarter of kicker cross section. . . . . . . 53

4.3 Kicking field along the central transverse line parallel to stripline. . . 54

4.4 The odd TEM mode and the even TEM mode of the kicker. . . . . . 55

4.5 Odd TEM mode along stripline. . . . . . . . . . . . . . 56

4.6 Even TEM mode along stripline. . . . . . . . . . . . 56

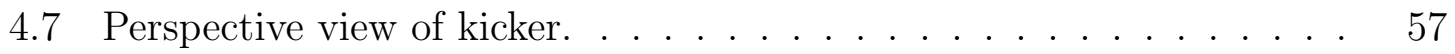

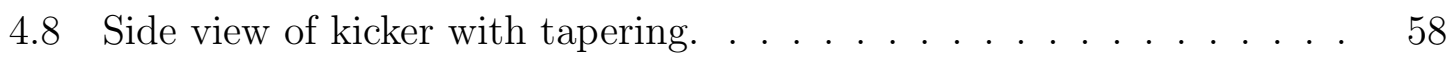

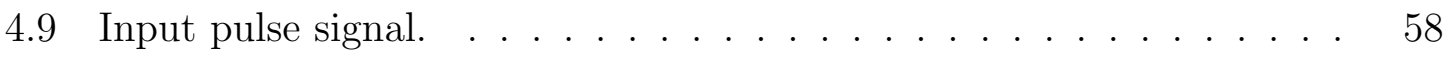

4.10 DFT of the input signal. . . . . . . . . . . . . . . 59

4.11 The scattering parameters between pulse port and dummy load port. 59

4.12 Electric field along the transverse center of kicker. . . . . . . . . 60

4.13 Electric field along the beam line center of kicker. . . . . . . . . 60

4.14 Time evolution of input, output and reflected signal . . . . . . . . 61

4.1 The sketch structure and equivalent circuit of the WGM . . . . . 64

4.2 Time response of WGM signal . . . . . . . . . . . . 65 
4.3 Ferrite torus and RF absorber of WGM . . . . . . . . . . 66

4.4 Resistors around the ceramic gap in WGM . . . . . . . . . . 67

4.5 The picture of the WGM bench test setup. . . . . . . . . . 68

4.6 WGM bench test signal . . . . . . . . . . . . . . . . . . . . . 69

4.7 WGM signal sensitivity to the beam position and orientation . . . . 70

4.8 Linear fitting in calibrating $\mathrm{R}$ of WGM $\ldots \ldots \ldots \ldots \ldots$

4.9 The WGM droop time measurement. . . . . . . . . . . . 71

4.10 The ALPHA injection beamline layout from LINAC to WGM. . . . . 73

4.11 WGM signal of injected beam without amplifier. The yellow trace is the beam current measurement, the red trace is the FFT of the yellow trace. . . . . . . . . . . . . . . . . . . . 74

4.12 WGM signal of injected beam with amplifier. . . . . . . . . . 74

4.13 Pseudo signal constructed in Matlab to simulate the LINAC beam pulse 76

4.14 The pseudo signal after the WGM bandwidth limit cutoff. . . . . . 77

4.15 The pseudo signal after the modulation and demodulation $\ldots \ldots 78$

4.16 Example 1: the pseudo signal and the processed signal. . . . . . . . 79

4.17 Example 2: the pseudo signal and the processed signal. . . . . . . . 79

4.18 Example 3: the pseudo signal and the processed signal. . . . . . . . 80

4.19 The relation between $r$ and the pulse width $\sigma \ldots \ldots \ldots$

4.20 The processed signal after modulation-demodulation. . . . . . . . 82

4.21 The injection beamline constructed in G4beamline. . . . . . . . . 84

4.22 The energy spectrum of the LINAC beam. . . . . . . . . . . 85

4.23 The comparison of nominal energy and the average beam energy at WGM location . . . . . . . . . . . . . . . . 85

4.24 The energy spectrum recovery from dipole scanning. . . . . . . . . 86

4.25 The energy spectrum of the LINAC beam. . . . . . . . . . 87 
4.26 The 2D projection of the longitudinal phase space of the macro beam pulse. . . . . . . . . . . . . . . . . . . . 88

4.27 The 3D painting of the longitudinal phase space of the macro beam

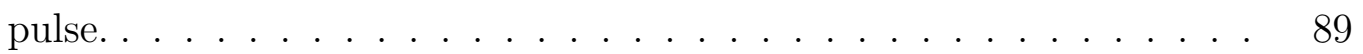

4.28 The frequency spectrum of the injected and extracted beam. . . . . . 90

5.1 Horizontal twiss parameters along the ring. $\alpha_{x}$ and $\beta_{x}$ are the CourantSnyder parameters; $D_{x}$ and $D_{p x}$ are the dispersion function and its derivative. . . . . . . . . . . . . . . . . . . . . 98

5.2 Vertical twiss parameters along the ring. $\alpha_{y}$ and $\beta_{y}$ are the CourantSnyder parameters; $D_{y}$ and $D_{p y}$ are the dispersion function and its derivative. . . . . . . . . . . . . . . . . . . . . . . 99

5.3 Horizontal emittance with different beam energies . . . . . . . . . . 104

5.4 Horizontal emittance with different beam currents . . . . . . . . . 105

5.5 Vertical emittance with different beam currents . . . . . . . . . . 106

5.6 Horizontal and vertical emittance with different coupling constant $\kappa$. 108 


\section{Chapter 1}

\section{Introduction}

Accelerator science began after Rutherford discovered the atomic structure by scattering $\alpha$ particles from radioactive nuclei with gold foil in 1911 [1]. In the past 100 years of development, the pursuit of ever increasing beam energy and beam intensity has lead to today's enormous sized, multibillion dollar accelerators like Large Hadron Collider (LHC) [2], Spallation Neutron Source (SNS) [3], Next Generation Light Source (NGLS) [4], and a possible muon collider [5, 6, 7, 8], built with cutting edge techniques and serving as the platform for the most frontier science research. In recent decades, another trend of smaller scale, more affordable accelerators has started to bloom in both academics and industry, with applications in more and more disciplines, including radiological medical treatment, neutron scattering studies, isotope

production, etc. For these accelerators, the most important issues are functionality, efficiency and long-term reliability.

Advanced eLectron-PHoton fAcility (ALPHA) is an example of a compact accelerator with applications in radiation effect testing [9]. The electron beam produced by ALPHA will be used to bombard electronic chips to test their performance in high radiation environments. By June 2011, major construction work on ALPHA has 
been finished and single pass mode operation has been successfully carried out. At the time of this writing, the machine upgrade for accumulation mode is underway and the commission work will resume in September.

Starting with a brief introduction to ALPHA, this thesis presents the work on ALPHA instrumentation and the study of beam dynamics. It shows, in extensive details, how accelerator science is applied in a novel way to provide new solutions beyond the conventional.

Chapter 2 introduces the design concept of ALPHA and how this design satisfies the performance requirements of radiation testing, as well as some beam physics studies to be carried out using ALPHA.

To overcome the Touschek effect and increase the beam lifetime in ALPHA, a first harmonic cavity needs to be installed. In Chapter 3, the refurbishing of Cooler Injector Synchrotron (CIS) RF cavity, a discussion of its performance limit due to the ferrite material and the design of a new cavity are presented.

The design of a fast extraction kicker is discussed in Chapter 4. The rise time of the kicker is required to be no more than $10 \mathrm{~ns}$. Considering such a fast rise time, as well as the high voltage requirement, a traveling wave stripline kicker is chosen.

For the beam diagnosis in the longitudinal direction, we have refurbished a wideband Wall Gap Monitor (WGM) from CIS. The WGM refurbishing and beam measurement are presented in Chapter 4. Proper shielding and amplifying are required to get an acceptable WGM signal. By comparing the injected and extracted beam signals in single pass commissioning, we have verified the debunching effect of the ALPHA storage ring. A modulation-demodulation signal processing method is developed to measure the current and longitudinal profile of the injected beam. By scanning the dipole strength in the injection line, we have reconstructed the tomography of longitudinal phase space of the LINAC beam.

In accumulation mode, ALPHA is designed to store a uniform beam with 50 
ns length, $50 \mathrm{MeV}$ energy and current up to $20 \mathrm{~A}$. Under such a low energy, high current condition, intra beam scattering (IBS) becomes a dominant effect on the beam emittance. In Chapter 5, a self-consistent simulation, including the IBS effect, the gas scattering and the linear coupling, is carried out to estimate the emittance of the storage beam.

The final conclusion is given in Chapter 6 . 


\section{Chapter 2}

\section{Introduction to ALPHA}

ALPHA, Advanced eLectron-PHoton fAcility, is a low energy compact electron accelerator under construction and being commissioned in the Indiana University Center for Exploration of Energy and Matter (CEEM). It is mainly composed of a LINAC injector and a 20 meter storage ring, as shown in Figure 2.1. The mission of ALPHA is to provide an electron beam for radiation effect testing and to serve as a platform for beam physics study.

In this chapter, we will introduce the design of the ALPHA storage ring [10], the radiation effect testing and the beam physics studies to be carried out at ALPHA.

\subsection{ALPHA storage ring design concept}

The ALPHA storage ring is composed of four 90 degree bending dipoles, each with an edge angle of 12 degree. The four bending dipoles provide horizontal focusing and the dipole edge angles provide vertical focusing. There is no extra quadrupole in the ring for beam focusing.

With only these four dipoles, the matchine cannot serve as a storage ring due to its 


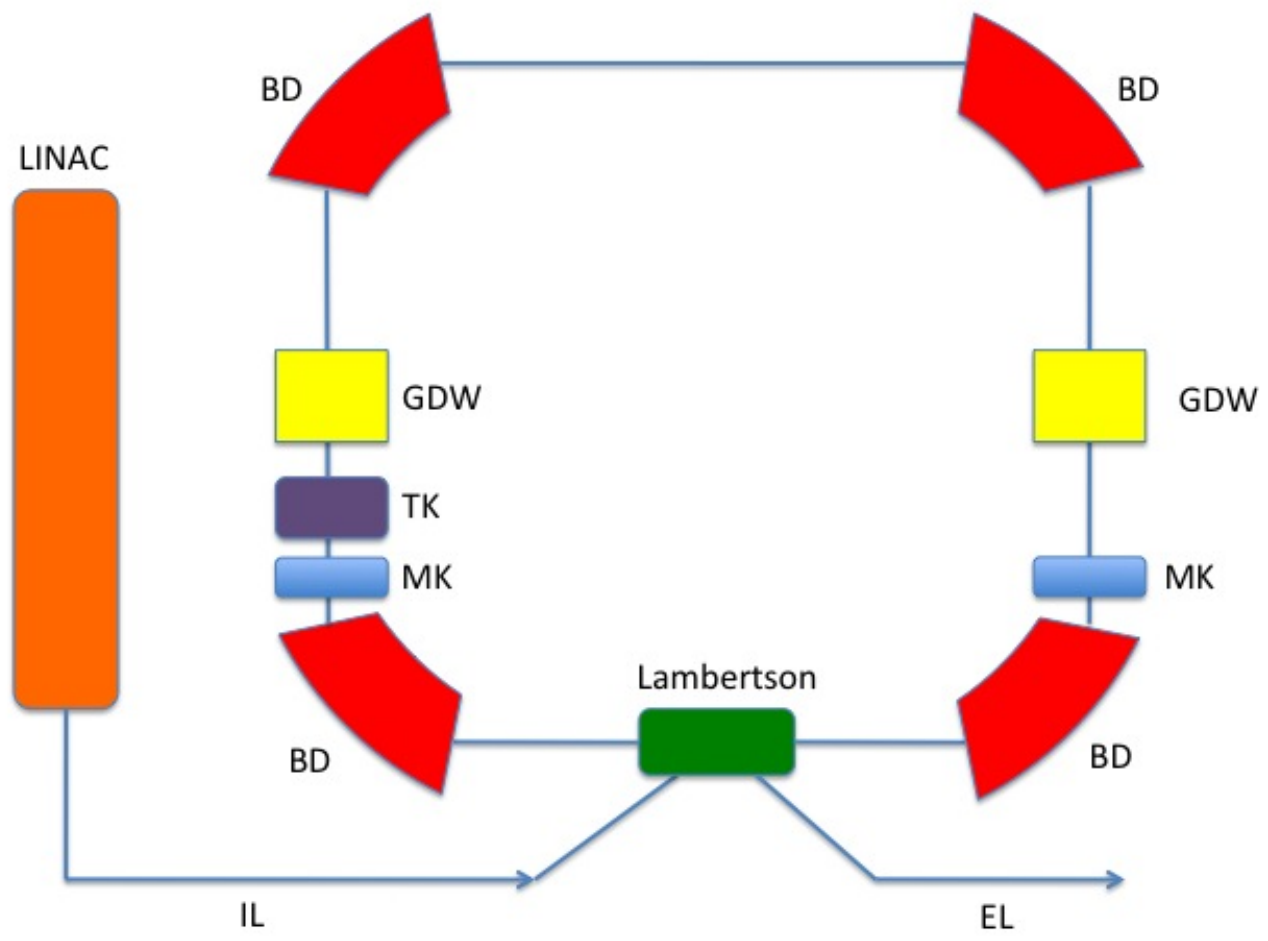

Figure 2.1: Simplified layout of ALPHA. BD: bending dipole, GDW: gradient damping wiggler, TK: traveling wave kicker, MK: magnetic kicker, IL: injection line, EL: extraction line. 
negative damping partition number $J_{x}$, which indicates that the horizontal emittance will grow until the beam strikes the vacuum chamber. To adjust $J_{x}$ from negative to positive and obtain a stable stored beam, two gradient damping wigglers (GDW) are installed in the ring, as seen in Figure 2.1.

Besides $J_{x}$, the GDW can also change the momentum compaction factor $\alpha_{c}$ of the ring. At a certain strength, the GDW can tune $\alpha_{c}=0$, thus the ring becomes quasi-isochronous. As $\alpha_{c}$ is a vital parameter for the LINAC beam debunching in the single pass mode of radiation effect testing, the GDW can adjust the debunching performance of the storage ring.

Two magnetic kickers are placed 90 degrees apart around the Lambertson to bump the closed orbit into the Lambertson field region for both injection and extraction. A traveling wave kicker is placed about 90 degrees ahead of the Lambertson to extract the entire storage beam in one turn.

\subsection{Radiation effect testing}

In the radiation effect testing, ALPHA will be operated in two modes: single pass mode and accumulation mode. In single pass mode the beam is injected into the ring, circulates one turn and is extracted. This mode debunches the microstructure of the LINAC beam and provides a uniform beam for radiation effect testing.

Due to the RF accelerating field in the LINAC cavity, the electron beam is bunched into micropulses with a pulse width of a few pico seconds. The time interval between micropulses is related to the RF frequency of the LINAC. For the Varian medical LINAC used in the ALPHA machine commissioning, the LINAC RF frequency is $2.856 \mathrm{GHz}$, so the period of the micropulse is about $350 \mathrm{ps}$.

The RF signal carried by the micropulse structure will interfere with the signal of the electronics under testing. To eliminate this interference due to the micropulse 
beam, we utilize the momentum compaction effect of the storage ring. Electrons with different momenta have different path lengths when passing through the storage ring. In addition, the velocity of all the electrons in ALPHA can be regarded as the speed of light. Thus electrons with different momenta will spread in the longitudinal direction and the beam becomes debunched after passing through the storage ring, as seen in Figure 2.2. The momentum compaction effect is described by the momentum compaction factor $\alpha_{c}$ :

$$
\Delta C / C_{0}=\alpha_{c} \times \delta
$$

where $\Delta C$ is the change of path length, $C_{0}$ is the close orbit length and $\delta$ is the relative momentum spread. When $\left|\alpha_{c}\right|$ is large enough, the micropulse can be debunched in just one turn.

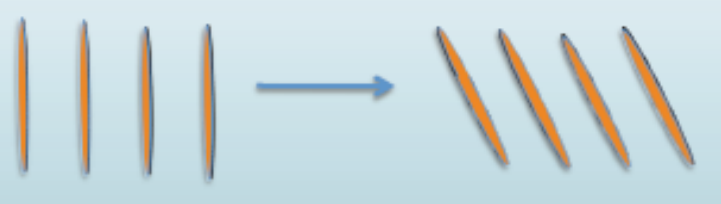

Figure 2.2: Beam debunching illustration. For $\alpha_{c}>0$, electrons with larger momenta have longer path lengths and thus lag behind after passing through one turn. Electrons with smaller momenta have shorter path lengths and advance forward. In this way, the micropulse is stretched out in longitudinal phase space. When $\left|\alpha_{c}\right|$ is large enough, the micropulse can be debunched in just one turn.

The debunching effect can be characterized quantitatively by a debunching factor $D$ :

$$
D=\frac{I_{\max }-I_{\min }}{I_{\max }+I_{\min }}
$$


When the beam is totally bunched, $I_{\min }=0$, thus $D=1$. If the beam is completely debunched, $I_{\max }=I_{\min }$ and $D=0$.

In the accumulation mode, the LINAC injects the beam into the storage ring continuously. The beam is stored in the ring until enough charge has been accumulated. Then all the stored beam will be extracted out in just one turn. This mode is to provide a large and uniform radiation dose for the testing.

In the extraction line, two octupoles are installed to perform nonlinear beam spreading (NBS). In NBS, a gaussian profile beam is folded into a uniform rectangular profile beam without any beam loss. Compared with the traditional masking technique, this method provides a better confined and more uniform beam with higher efficiency for radiation testing.

\subsection{ALPHA beam physics study}

Many interesting beam physics studies can be carried out using ALPHA. Below are some examples.

\subsubsection{Intrabeam Scattering}

In accumulation mode, the stored beam is of high current and low energy. Under such condition, intrabeam scattering (IBS) is a dominant factor on the beam emittance. The IBS effect depends sensitively on peak beam current, energy, emittance and lattice parameters. Details of IBS simulations will be deferred until Chapter 5. In ALPHA, experiments can be carried out to study how each factor contributes to IBS effect and compared with the simulation result. 


\subsubsection{Touschek Life Time}

As a result of internal coulomb scattering, as in IBS, the Touschek effect is a major concern for a low energy and high current storage ring such as ALPHA. The detailed discussion on Touschek effect is in Chapter 3. Different from the multiple small angle coulomb scattering in IBS, Touschek effect is a large angle coulomb scattering and the particles are lost in single collisions. Tuning different parameters of the beam and the RF voltage, one can study how these factors effect the Touschek life time and compare the experimental measurements with previous simulations [10].

\subsubsection{Beam manipulation by Gradient Damping Wiggler}

As mentioned before, by tuning the GDW strength one can tune the momentum compaction factor $\alpha_{c}$ and thus tune the debunching factor $D$ of the ALPHA storage ring. The value of $D$ can be calculated from the beam frequency spectrum measured by a wall gap monitor. Thus one can study the GDW tuning function experimentally. The GDW can also change the momentum compaction factor $\alpha_{c}$ and tune the ring into isochronous with $\alpha_{c}=0$. Thus one can study the beam behavior under isochronous condition. 


\section{Chapter 3}

\section{The RF Cavity}

In accelerators, Radio Frequency $(\mathrm{RF})$ cavities provide longitudinal electric field to accelerate or decelerate the beam. In the 3rd generation synchrotron light source of GeV energy level, like the Advanced Light Source in Lawrence Berkeley National Lab and the Advanced Photon Source in Argonne National Lab, the RF cavity is used to compensate the energy loss caused by the synchrotron radiation. In the storage ring of $\mathrm{MeV}$ energy level, like ALPHA, the synchrotron radiation becomes less important and the requirement of energy replenish is not significant. However, the Touschek scattering will cause the beam loss in the longitudinal phase space. In this case, the RF Cavity is used to provide the longitudinal focusing to overcome the Touschek effect, thus to increase the life time of the stored beam.

\subsection{Introduction of RF Cavity}

RF Cavity is a device that can hold high electric field with minimum energy loss. Classified by geometry, there are pill box cavity, quarter-wave cavity, drift-tube cavity, coupled side cavity, etc. Classified by the electric field for accelerating the beam, there 
are standing wave cavity and traveling wave cavity. Classified by cavity material, there are normal conducting RF cavity and superconducting RF cavity.

In general, RF cavity is an instrumentation that establishes a RF oscillating field in a confined region along the beam path. Every time beam passes this region, it interacts with the RF electric field and gets accelerated or decelerated.

Several figures of merit are used to characterize a RF cavity.

\section{Harmonic number $h$}

For a synchrotron with revolution frequency $f_{0}$, the RF frequency of the cavity is synchronized with the beam at:

$$
f=h \times f_{0}
$$

where $h$ is an integer named harmonic number. Only when $h$ is an integer that the cavity accelerating field can exert on the revolution beam coherently.

2. Transit time factor $F_{t}$

Assuming the accelerating field varies sinusoidally with time

$$
E_{z}(s, t)=E_{z}(s) \sin (\omega t)
$$

and the accelerating gap width is $g$, the transit time factor is defined as:

$$
F_{t}=\frac{\left.\mid \int E_{z}(s)\right) \cdot e^{j \phi(t)} \cdot d s \mid}{\int\left|E_{z}(s)\right| \cdot d s}
$$

The transit time factor reflects that the beam needs certain time to traverse through the RF cavity, and the accelerating field is varying in this time. With $F_{t}$, the energy gain of the beam after passing through the cavity can be expressed as:

$$
\Delta W=q V_{0} \cdot F_{t} \cdot \cos \phi_{p}
$$


where

$$
V_{0}=\int\left|E_{z}(s)\right| \cdot d s
$$

and

$$
\phi_{p}=\arctan \left(\frac{\int E_{z} \cdot \sin \phi(t) \cdot d s}{\int E_{z} \cdot \cos \phi(t) \cdot d s}\right)
$$

Usually $F_{t}$ and $\phi_{t}$ are complicated and calculated by numerical simulation code. Under the approximation that $E_{z}$ is a constant across the gas and zero anywhere outside the gap, $F_{t}$ is simplified to be:

$$
F_{t}=\frac{\sin \left(h g / 2 R_{0}\right)}{h g / 2 R_{0}}
$$

where $h$ is the harmonic number, $g$ the gap width and $R_{0}$ the radius of bending dipole.

3. Quality factor $Q$

The quality factor is defined as the ratio between the energy stored in the cavity and the energy dissipated in one RF cycle.

$$
Q=\frac{E_{\text {stored }}}{E_{\text {dissipated }} \text { in one RF cycle }}
$$

The energy stored in the cavity is mainly concentrated around different resonant frequencies. For each resonant frequency, there is a corresponding $Q$ value. The $Q$ value of the cavity is often referred to the resonant frequency of the accelerating mode.

4. Shunt impedance $R_{s}$

The shunt impedance characterizes the accelerating efficiency of the cavity:

$$
R_{s}=\frac{V_{R F}^{2}}{2 P_{d}}
$$


where $V_{R F}$ is the peak voltage across the cavity and $P_{d}$ is the average dissipated power in the cavity. For a given RF input power, the larger the $R_{s}$, the higher the $V_{R F}$ across the gap

The ratio $\frac{R_{s}}{Q}$ is mainly determined by the geometry of the cavity, regardless of cavity wall material or input power. It describes the relation between the total energy stored in the cavity and the energy distributed around the accelerating gap in the form of $V_{R F}$.

The revolution frequency of ALPHA storage ring is $15 \mathrm{MHz}$. If the cavity operates at $h=1$, its principle resonant frequency should also be $15 \mathrm{MHz}$. For such a low resonant frequency, and with a reasonable compact size, the ferrite loaded cavity is the first choice.

\subsection{Touschek Effect}

Coulomb scattering of charged particles in a stored beam causes an exchange of energies between the transverse and longitudinal motion. It changes, therefore, the betatron and synchrotron oscillation coordinates of the colliding particles. One consequence is the Touschek effect which is the transformation of a small transverse momentum into a large longitudinal momentum due to the scattering. Then both scattered particles are lost, one with too much and one with too little energy. The amplification of the momentum change is a relativistic effect so that the change of the longitudinal momentum is increased by the Lorentz factor $\gamma$.

The number of particle lost per unit time is given by:

$$
\frac{d N}{d t}=-R=-a N^{2}
$$

where $R$ is the loss rate and $a$ is a constant. The number of particle at time $t$ can be 
expressed as [11]:

$$
N=\frac{N_{0}}{1+N_{0} a t}
$$

where $N_{0}$ is the number of particles at time $t=0$. The Touschek life time $T_{l}$ can be defined as:

$$
\frac{1}{T_{l}}=\left\langle a N_{0}\right\rangle=\left\langle\frac{R}{N_{0}}\right\rangle
$$

The calculation of $T_{l}$ for ALPHA is done in [10]. From this calculation, we decided on a $1 \mathrm{kV}$ minimum $\mathrm{RF}$ voltage requirement on ALPHA cavity.

\subsection{Refurbishing of CIS RF Cavity}

The Cooler Injector Synchrotron (CIS) is a proton booster that used to operate at Indiana University Cyclotron Facility (IUCF). Since CIS stopped commissioning in the early 2000s, the CIS cavity has stayed in storage. It is a ferrite loaded quarterwave like cavity with co-axial structure. Its resonant frequency can be tuned from 2 $\mathrm{MHz}$ to $10 \mathrm{MHz}$, which is close to the ALPHA cavity frequency $15 \mathrm{MHz}$. Thus we tried to refurbish the CIS Cavity for ALPHA, which can save both money and time. The goal of the refurbishing is:

1. Tuning the resonant frequency to $15 \mathrm{MHz}$, with good power transmission efficiency.

2. Achieving at least $1 \mathrm{KV}$, ideally $3 \mathrm{KV}$ gap voltage in high power test.

3. Studying the characteristics of the Cavity. In case the refurbishing plan doesn't work, we can still benefit from this experience to design a new cavity for ALPHA. 


\subsubsection{The CIS RF Cavity}

The structure of the CIS RF cavity is shown in Figure 3.1

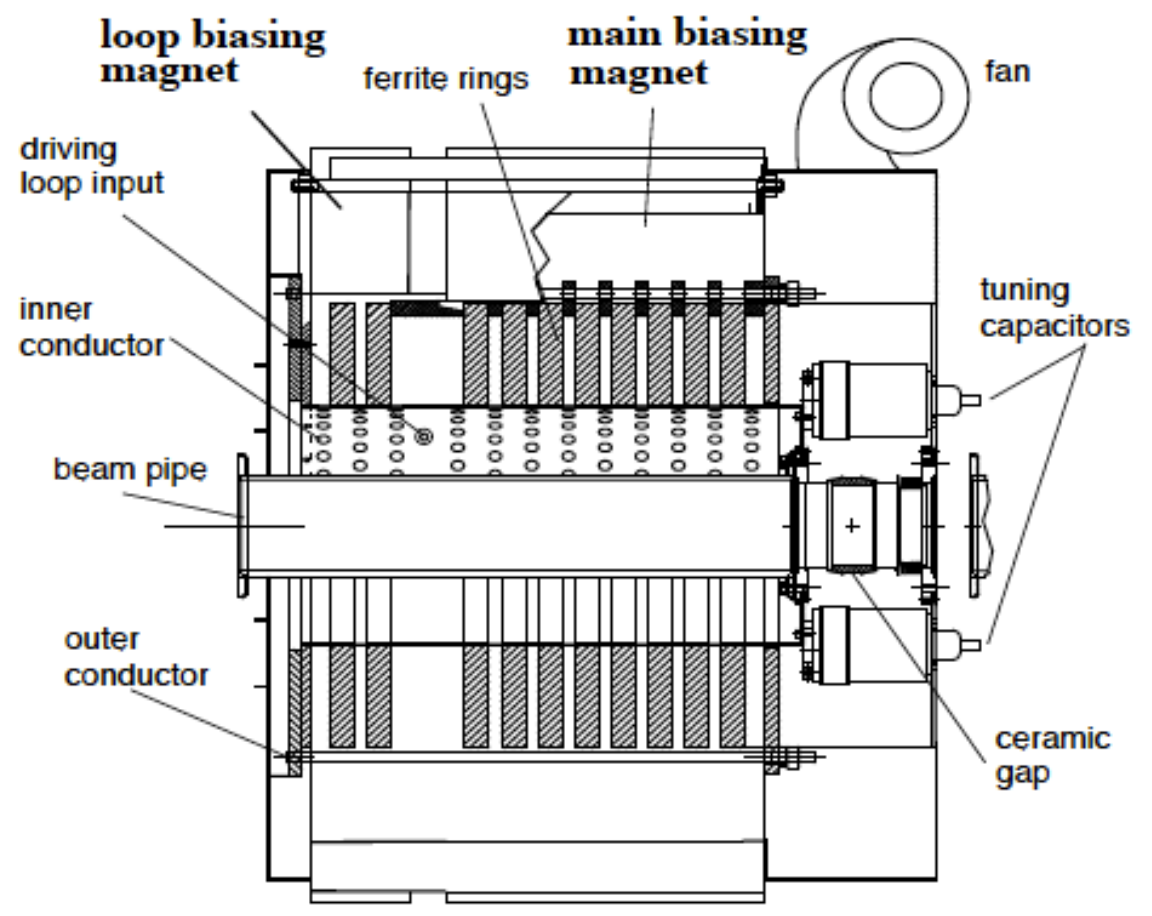

Figure 3.1: The Structure of the CIS cavity

It is a quarter-wave like coaxial cavity, with a ceramic gap at about $1 / 4$ wavelength position. There are 10 ferrite disks inside the cavity, grouped into the loop biasing magnets and main biasing magnets. The main biasing magnets are mainly for tuning the resonant frequency and the loop biasing magnets are mainly for impedance match. An external tunable capacitor is installed across the ceramic gap to expand the frequency tuning range.

The revolution frequency of the proton beam in CIS was from about $2 \mathrm{MHz}$ to $10 \mathrm{MHz}$ during the energy ramping. Thus as a first harmonic cavity, the resonant 
frequency of CIS RF cavity should also be tuned from $2 \mathrm{MHz}$ to $10 \mathrm{MHz}$, keeping consistent with the beam revolution frequency.

\subsubsection{The Frequency Tuning and impedance matching of CIS RF Cavity}

The frequency tuning of CIS RF cavity is done by adjusting the bias current on the quadrupole magnet outside the cavity, and by adjusting the external capacitor. The detailed discussion of the tuning mechanism can be found in [12]. In order to refurbish the CIS cavity for ALPHA, the first task is to tune the resonant frequency to $15 \mathrm{MHz}$, which is the revolution frequency of ALPHA.

In our frequency tuning experiments, the resonant frequency is measured by a Network Analyzer (NWA). The power input port of the cavity is connected to the NWA and the reflection coefficient $S_{11}$ is measured. The resonant frequency, which is tuned mainly by the main biasing current, is the frequency corresponding to the minimum value of $S_{11}$. To achieve the most efficient power feeding, we also want to match the impedance from the power cable to the cavity coupling port. The effective impedance at the cavity coupling port is tuned mainly by the loop biasing current. The power input efficiency is measured by the Standing Wave Reflection (SWR) from NWA, which is defined as:

$$
\mathrm{SWR}=\frac{1+\left|S_{11}\right|}{1-\left|S_{11}\right|}
$$

The SWR should be 1 when there is no reflected power, otherwise it will be larger than 1 . In the reality it is hard to tune SWR exactly to 1 but we always try to make it as small as possible.

At a fixed external capacitance value, we sweep the main bias current from $0 \mathrm{~A}$ to $15 \mathrm{~A}$ and measure the resonant frequency. The loop bias current is tuned to make SWR as low as possible all the time. The tuning result is shown in Figure 3.2. The 
resonant frequency has achieved $15 \mathrm{MHz}$ with a $12 \mathrm{~A}$ main bias current and a $10 \mathrm{~A}$ loop bias current.

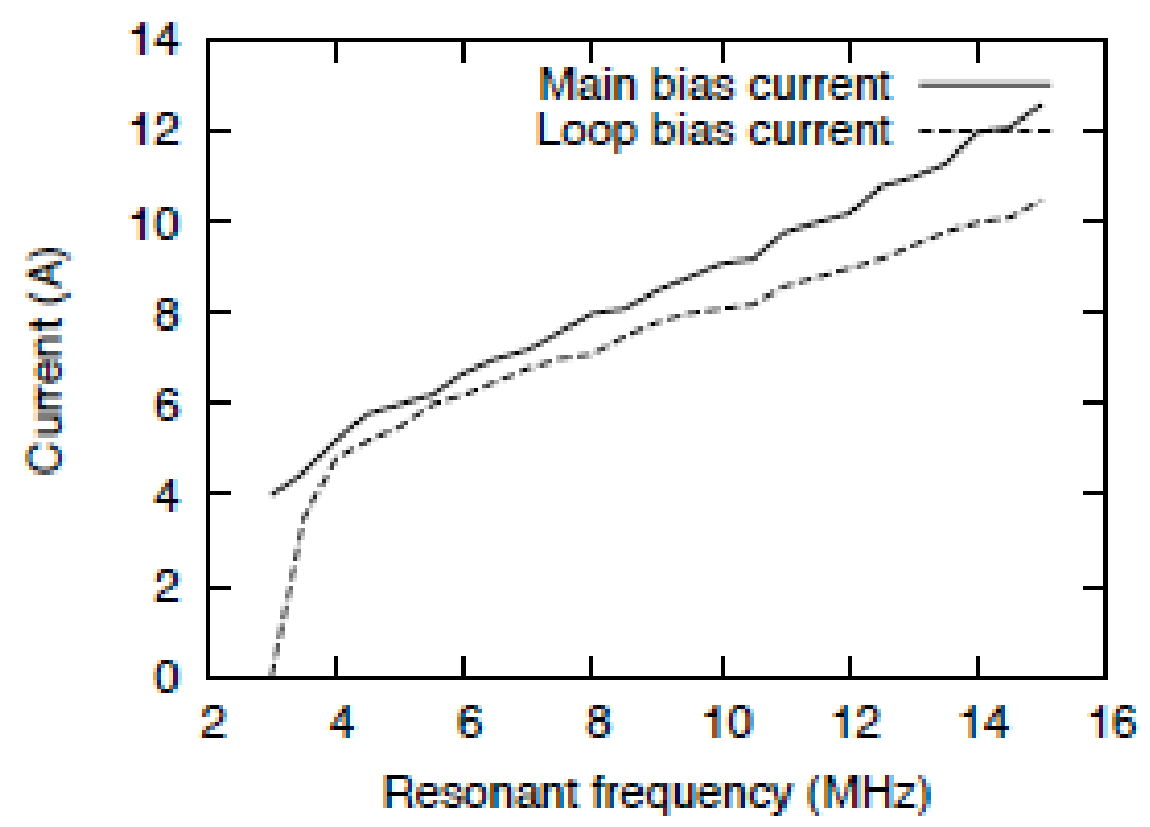

Figure 3.2: Bias Current versus resonant frequency

During the tuning, we find that the resonant frequency and SWR become more and more sensitive to the bias currents as they increase. Because the current is adjusted mechanically by human hand, and the current reading from the scale can't be very accurate, $10.5 \mathrm{~A}$ and $12.5 \mathrm{~A}$ are the rough values. Refine adjustment is needed around these current settings.

We have also noticed that the resonant frequency keeps on drifting up slowly after we turn on the bias currents. This is because that the heat dissipation of current wire will increase the temperature of the ferrite disks, thus the effective permeability of the ferrite decreases and the cavity resonant frequency increases. The Figure 3.3 shows 
how the resonant frequency drifts with time in three independent mesurements.

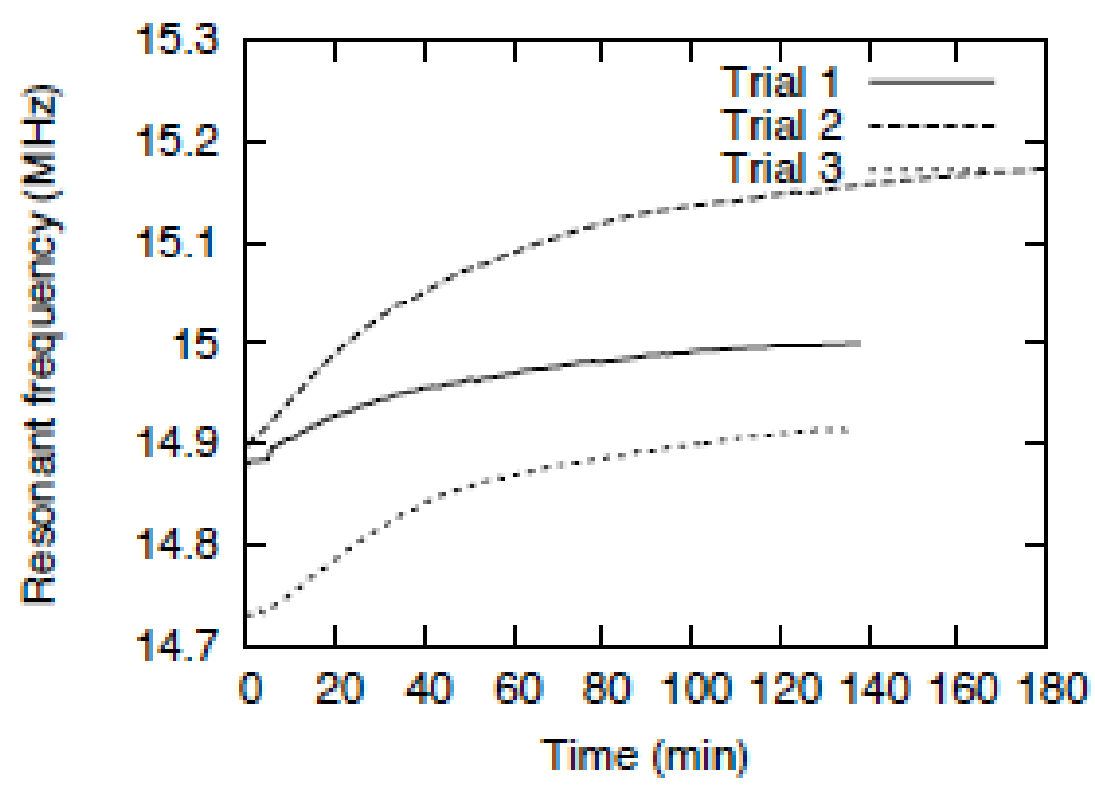

Figure 3.3: Resonant requency drift with time after tuning on the bias current of 3 independent trials.

To overcome this drifting instability, we can either install some feedback system to control the resonant frequency, or we can turn on the cavity to reach an equilibrium temperature and stable resonant frequency before the operation.

Besides the resonant frequency, the SWR also drifts with time. However this drift is very small and the system can be regarded as critically coupled all the time.

\subsubsection{The Quality Factor Q measurement}

We use two methods to measure the quality factor $Q$ of the cavity. 


\section{3db Method}

In the "3db method", we use the NWA to measure the transmission coefficient $S_{21}$. The port 1 of NWA is connected to the cavity power input port and the port 2 of NWA is connected to one of the external capacitor coupling port. From the data of S21, we can find the resonant frequency $f_{0}$ and the frequency interval between two $3 \mathrm{db}$ points $\Delta f$, the quality factor $Q$ :

$$
Q=\frac{f_{0}}{\Delta f}
$$

The measurement results is

$$
Q \approx 6.5
$$

Notice that during the measurement the cavity plays as a load of NWA, so this $Q$ is the loaded quality factor $Q_{L}$. The intrinsic quality factor $Q_{0}$ of the cavity itself, under the critical coupling, is twice of $Q_{L}$ [13]. So:

$$
Q_{0} \approx 13
$$

\section{Linear Fit Method}

In the "linear fit method", we use NWA to measure the reflection coefficient $\Gamma$, which is the reading of $S_{11}$ from NWA. This method is particularly suitable for the low-Q system, such as the CIS cavity. Around the resonant frequency, there is a linear relation:

$$
Q_{L} \delta=j\left(\frac{1+\Gamma_{0}}{1-\Gamma_{0}}-\frac{1+\Gamma}{1-\Gamma}\right) \frac{1-\Gamma_{0}}{2}
$$

where $\delta=\omega / \omega_{0}-\omega_{0} / \omega, \Gamma$ is reflection coefficient and $\Gamma_{0}$ is the reflection coefficient at resonant frequency [14].

Pay attention that we can't use the reflection coefficient we read directly from NWA for this fitting formula. Instead we need to rotate the $\mathrm{Q}$ circle on the smith 
chart to make $\Gamma_{0}$ to be real, then use the reflection coefficient from rotated Q circle, shown as in Figure 3.4.

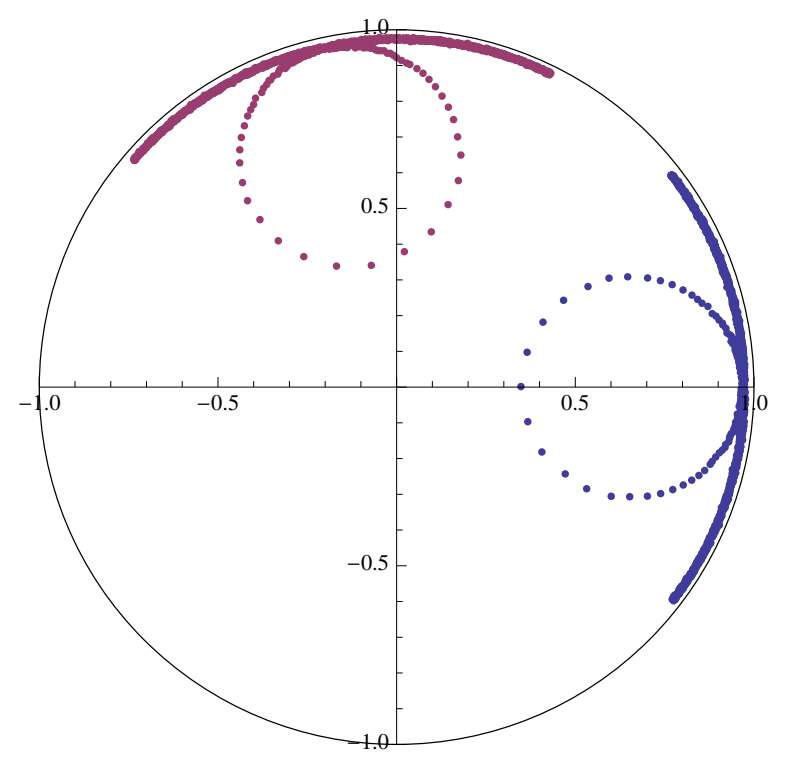

Figure 3.4: $\mathrm{Q}$ circle rotation on smith chart for linear fit. The red $\mathrm{Q}$ circle is the raw data and the blue is after rotation.

The fitting result is shown in Figure 3.5, where $X=\delta$ and $Y=\operatorname{Re}\left[j\left(\frac{1+\Gamma_{0}}{1-\Gamma_{0}}-\right.\right.$ $\left.\left.\frac{1+\Gamma}{1-\Gamma}\right) \frac{1-\Gamma_{0}}{2}\right]$. The slope is fitted to be 6.6 , which is the value of $Q_{L}$. So

$$
Q_{0}=2 Q_{L} \approx 13
$$

Thus the results from these two methods agree with each other.

For a heavy ferrite-loaded cavity, the quality factor $Q$ is mainly determined by the $Q$ value of the ferrite material itself. The energy loss in such a cavity is mainly from the loss in ferrite, including hysteresis loss and eddy current loss. They are much larger than the current heating loss in the cavity wall. Overall, the $Q$ value of ferrite cavity is lower than the cavities whose $Q$ values mainly depend on the energy loss in the cavity wall. 


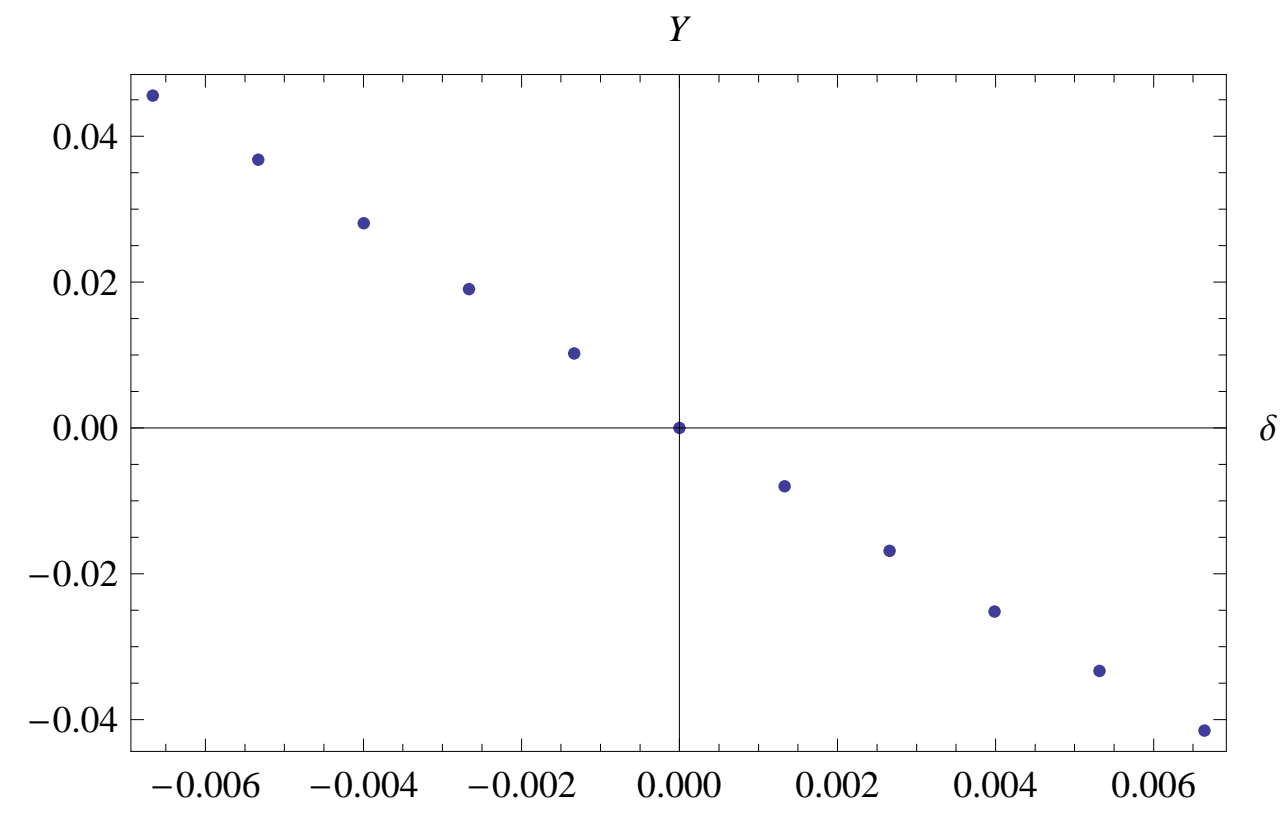

Figure 3.5: Linear-fit for $Q_{L}$

\subsubsection{Internal Capacitance Measurement}

The internal capacitance of the cavity $C_{0}$ comes from the co-axial structure and the ceramic gap. It is exclusively determined by the cavity geometry. In parallel with the ceramic gap, there is an external capacitor Jennings cvcd-2000-5s. It is tunable and according to the manual, its capacitance $C$ can be roughly expressed, in the unit of $\mathrm{pF}$, as:

$$
C=2000-\lambda N
$$

where $\mathrm{N}$ is the tuning turn number and $\lambda$ is about $111.11 \mathrm{pF} /$ turn. By tuning the external capacitance $C$, we can measure the internal capacitance $C_{0}$.

Considering the sum of internal and external capacitance, the resonant frequency of cavity can be expressed as :

$$
2 \pi f_{0}=\frac{a}{\sqrt{C+C_{0}}}=\frac{a}{\sqrt{2000-\lambda N+C_{0}}}
$$


where $a$ is a constant. From this we can derive a linear relation between $N$ and $\frac{1}{f_{0}}$ :

$$
N=\frac{b}{f_{0}^{2}}+\frac{2000+C_{0}}{\lambda}
$$

where $b$ is another constant. Thus by linear fitting $N-\frac{1}{f_{0}{ }^{2}}$ curve and calibrating the intersection, we can measure $C_{0}$.

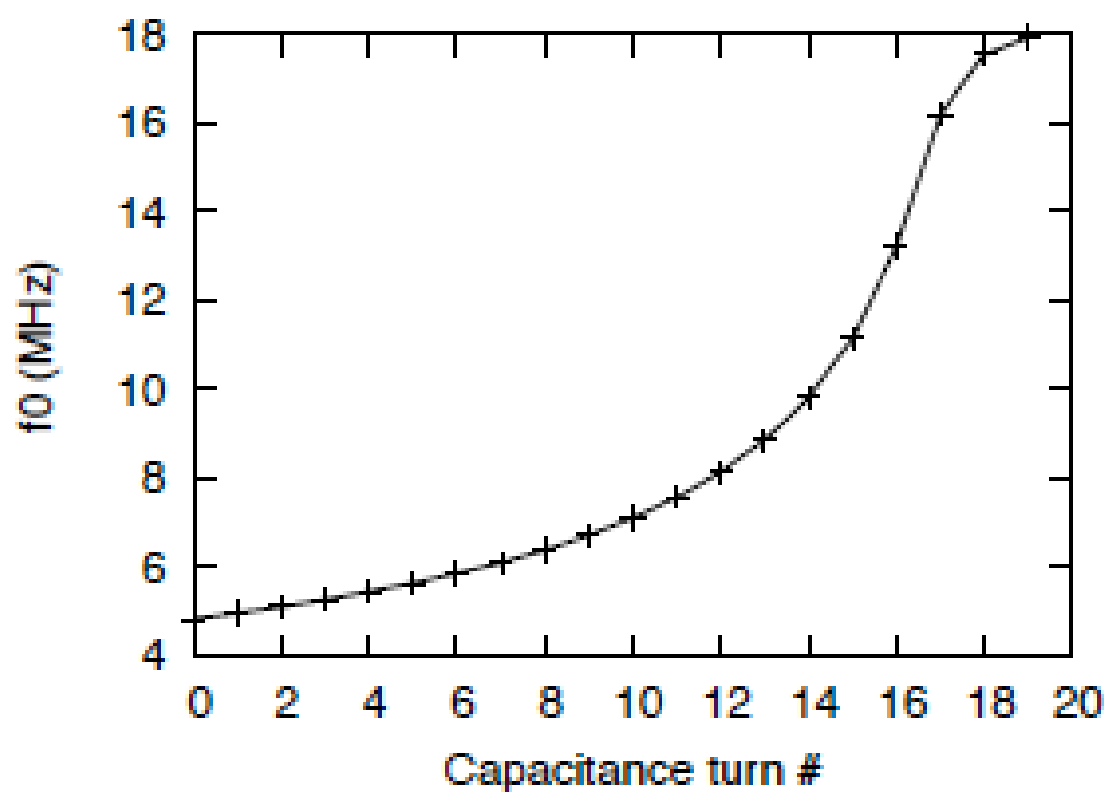

Figure 3.6: The resonant frequency tuning by external capacitor

The measurement of resonant frequency tuning by external capacitor is shown in Figure 3.6 and the $N-\frac{1}{f_{0}{ }^{2}}$ linear fitting is shown in Figure 3.7.

From the data fitting:

$$
\frac{2000+C_{0}}{\lambda}=18.533
$$

Thus we can calculate the internal capacitance of the cavity:

$$
C_{0}=59.22 \mathrm{pF}
$$




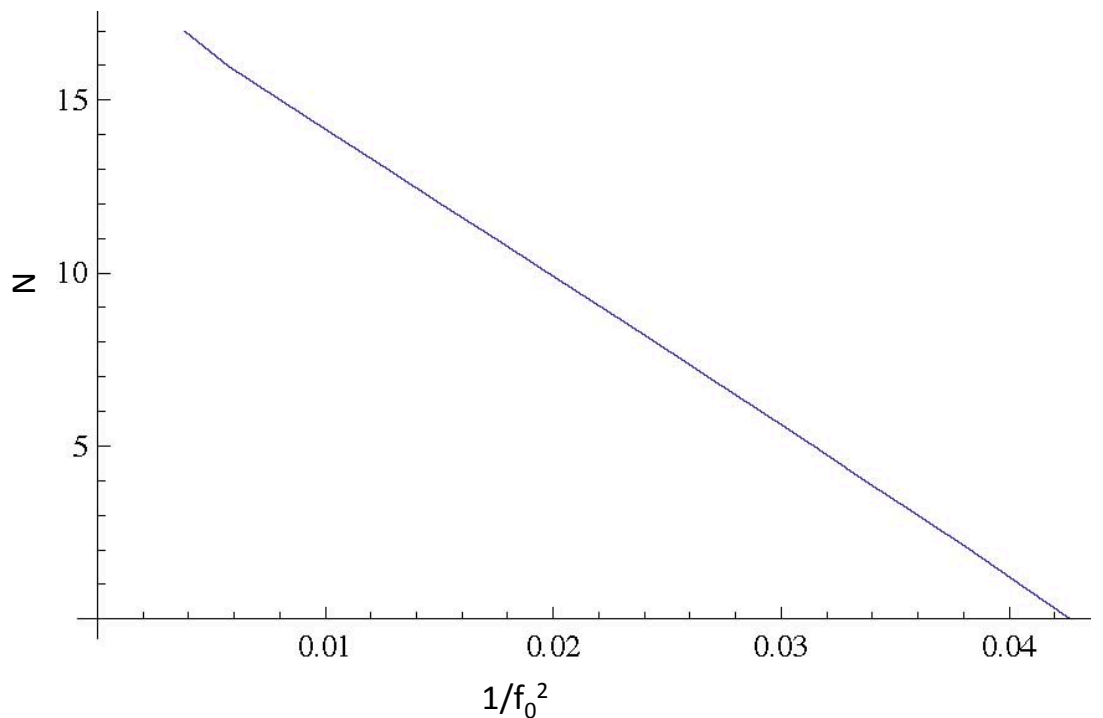

Figure 3.7: The linear fitting of $N-\frac{1}{f_{0}{ }^{2}}$ 


\subsubsection{The Shunt Impedance Measurement by Bead Pull}

For a RF cavity, the shunt impedance $R_{s}$ indicates the energy efficiency, as shown in its definition above. In order to measure $R_{s}$ of the cavity, we use the bead-pull method to measure $R_{s} / Q_{0}$. As we already measure $Q_{0} \approx 13$, we can calculate $R_{s}$ from the value of $R_{s} / Q_{0}$. The experiment set up is shown in Figure 3.8.
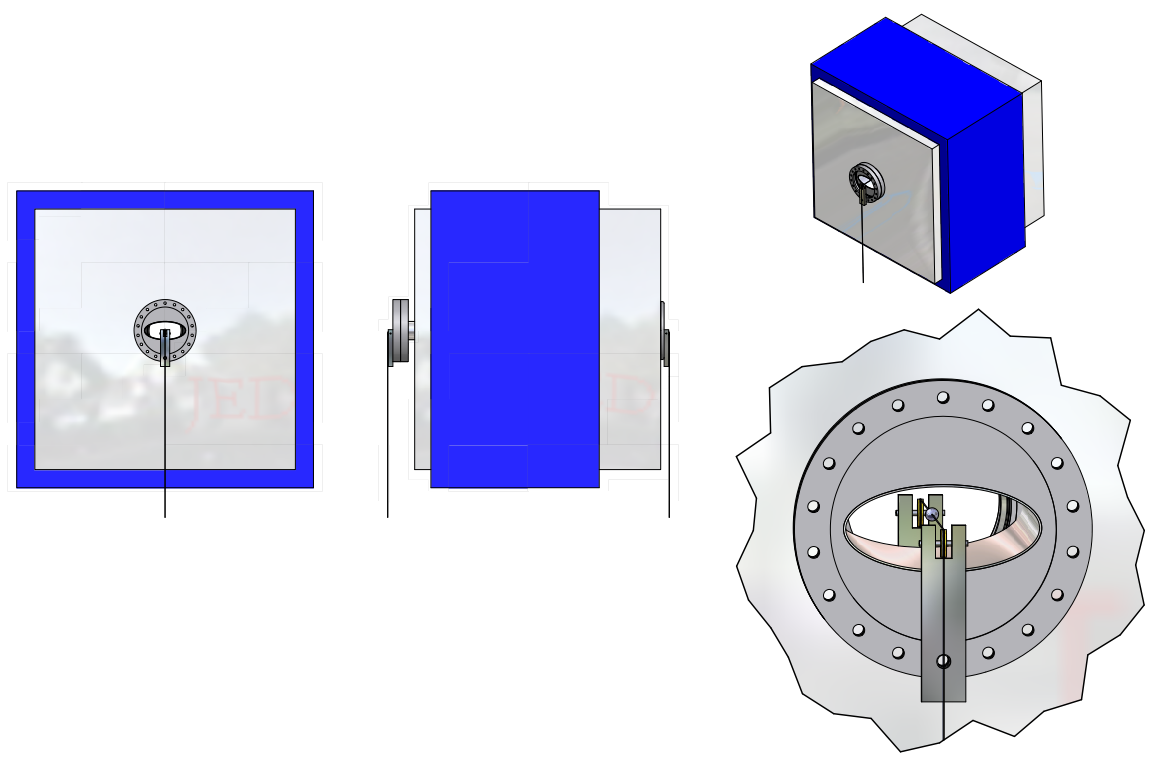

Figure 3.8: Bead-pull measurement set up, drawn by Jack Doskow

We used an aluminum bead with radius $r=0.9625 \mathrm{~cm}$. The volume of the bead is

$$
\Delta \tau=\frac{4}{3} \pi r^{3} \approx 3.733 \times 10^{-6} m^{3}
$$

While pulling the bead through the cavity, the electric field in the cavity will be disturbed, thus the resonant frequency of the cavity will shift. By measuring $S_{11}$ from NWA, we can monitor the frequency drift $\Delta f$ and calculate the $R_{s} / Q_{0}$ by the 
formula:

$$
\frac{R_{s}}{Q_{0}}=\frac{\epsilon_{r}+2}{\epsilon_{r}-1} \times \frac{\Delta f l^{2}}{\pi \epsilon_{0} f_{0}^{2} \Delta \tau}
$$

where the relative permittivity $\epsilon_{r}=9.3$ for aluminum, $l$ is the length of gap $\approx 0.04 m$ and $f_{0}$ is the resonant frequency $15 \mathrm{MHz}$. This formula has assumed that the electric field is concentrated in the accelerating gap and is almost homogenous. For our rough estimation, this assumption is acceptable. Otherwise one needs to use the more complicated integration form:

$$
\frac{R_{s}}{Q}=\frac{\epsilon_{r}+2}{\epsilon_{r}-1} \times \frac{1}{2 \omega_{0}} \times\left[\int_{g a p} \sqrt{\frac{\Delta \omega}{\omega_{0}}} d z\right]^{2}
$$

In this measurement, the frequency drift is as small as the frequency resolution of the NWA, which is $\Delta f=675 \mathrm{~Hz}$. The calculation of $R_{s}$ is as follows:

$$
\begin{aligned}
R_{s} & =Q_{0} \frac{\epsilon_{r}+2}{\epsilon_{r}-1} \times \frac{\Delta f l^{2}}{\pi \epsilon_{0} f^{2} \Delta \tau} \\
& =13 \times \frac{9.3+2}{9.3-1} \times \frac{(0.04)^{2} \times 675}{3.14 \times 8.85 \times 10^{-12} \times 225 \times 10^{1} 2 \times 3.733 \times 10^{-6}} \approx 819 \Omega
\end{aligned}
$$

Because the frequency drift $\Delta f$ is already as small as the minimum resolution of the NWA, we need to give it a $50 \%$ uncertainty, so:

$$
R_{s}=819 \Omega \pm 50 \%=[400 \Omega, 1200 \Omega]
$$

Considering the geometry factor due to the sphere shape of the bead, a more accurate formula for the bead perturbation is [15]:

$$
R_{s}=Q_{0} \times \frac{1}{2 \omega \pi r^{3} \epsilon_{0}} \times \frac{\epsilon_{r}+2}{\epsilon_{r}-1} \times \frac{\Delta \omega l^{2}}{\omega}
$$

This will give us a third of the previous result:

$$
R_{s}=(819 / 3) \Omega \pm 50 \%=273 \Omega \pm 50 \%=[137 \Omega, 410 \Omega]
$$

For a small Q value cavity, like this CIS ferrite loaded cavity, the bead pull method is not very suitable for the shunt impedance measurement. Due to the small Q, the 
voltage at the resonant frequency is not sharp thus it is harder to detect the resonant frequency shift. In order to get a more detectable frequency shift, one need to use a larger bead to provide larger perturbation. However, the whole theoretical basis of the bead pull method is rooted in the small perturbation assumption. A large perturbation may make this method no longer applicable. Thus for such a low $\mathrm{Q}$ cavity, we can only get a rough estimation of shunt impedance by bead pull method.

For the low Q cavity, the better way to measure the cavity efficiency is to do the high power test: power the cavity and measure the voltage across the accelerating gap directly. Figure 3.9 is the previous measurement of CIS cavity shunt impedance from $2 \mathrm{MHz}$ to $10 \mathrm{MHz}$. If we extrapolate this result up to $15 \mathrm{MHz}$, the impedance is about $310 \Omega$, which agrees well with the bead pull measurement.

\subsection{Transmission Line Analysis of Ferrite-Loaded Cavity}

The Transmission Line Analysis is an important analyzing method in RF engineering. In this method, the RF components are represented by the electric circuit elements and the E\&M field propagation is represented by the electric current flow, thus the calculation is much easier to carry out. Here we apply this method to analyze the ferrite-loaded cavity.

\subsubsection{The Ferrite in the co-axial cavity.}

The open-ended, co-axial metallic cylinder can be viewed as an inductor. The inductor formed with ferrite as a core material will exhibit an increase in inductance value compared with an air-cored version. There will also be energy loss in the ferrite core due to the hysteresis and eddy current. This loss can be represented by assigning a 


\section{CIS Cavity Frequency vs Shunt Impdance}

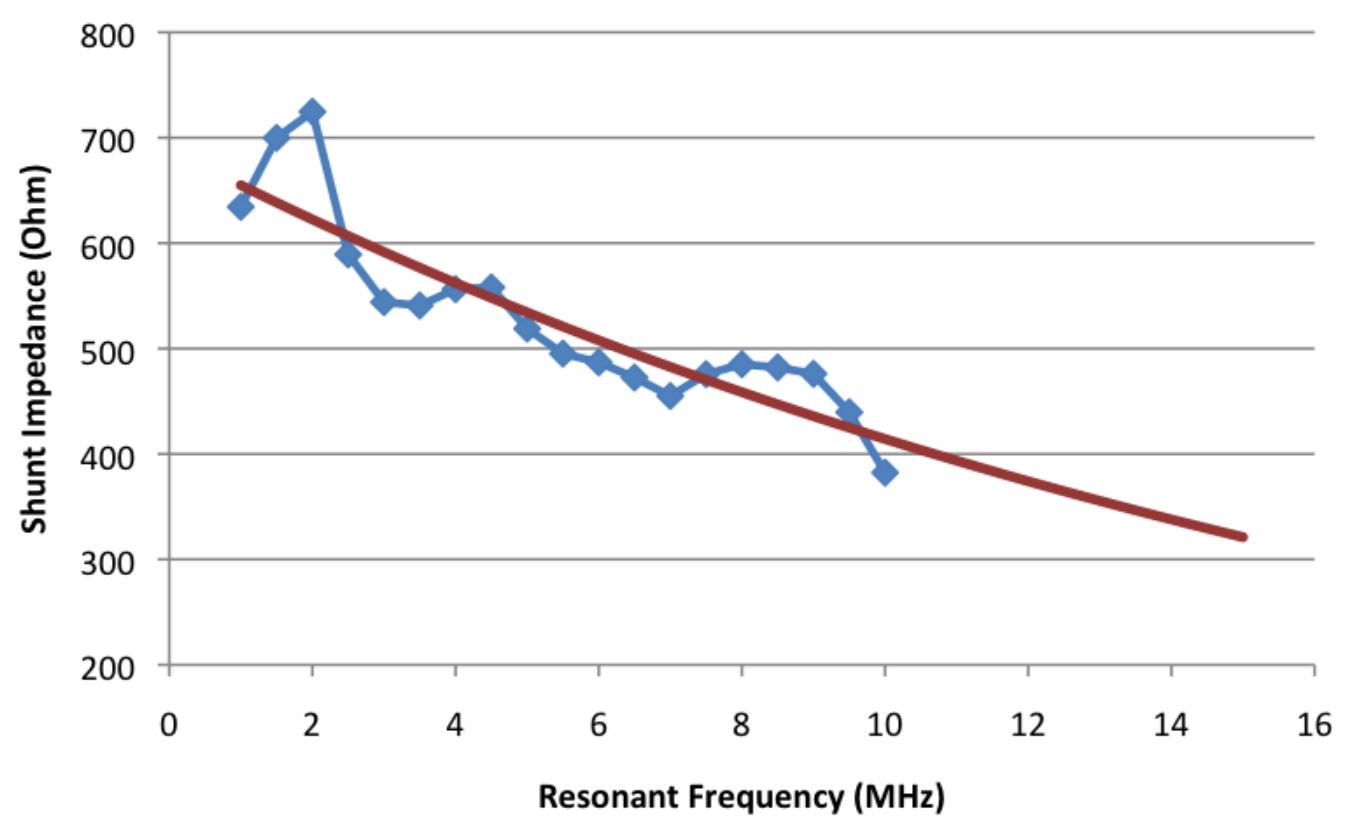

Figure 3.9: Shunt Impedance Measurement of CIS RF Cavity by high power test. The blue line and dots are the experiment data. The red line is the exponential fitting extrapolated to $15 \mathrm{MHz}$. From the extrapolation, we can estimate a $310 \Omega$ shunt impedance at 15 $\mathrm{MHz}$. 
complex value to the magnetic permeability such that:

$$
\mu=\mu^{\prime}-j \mu^{\prime \prime}
$$

The real part $\mu^{\prime}$ corresponds to the inductance and the imaginary part corresponds to ferrite core loss. Thus the impedance of the inductance is:

$$
\begin{aligned}
Z & =j \omega \mu L_{0} \\
& =j \omega L_{0}\left(\mu^{\prime}-j \mu^{\prime \prime}\right) \\
& =j \omega L_{0} \mu^{\prime}+\mu^{\prime \prime} \omega L_{0} \\
& =j \omega L+r
\end{aligned}
$$

where $L_{0}$ is the value of the air-cored inductance:

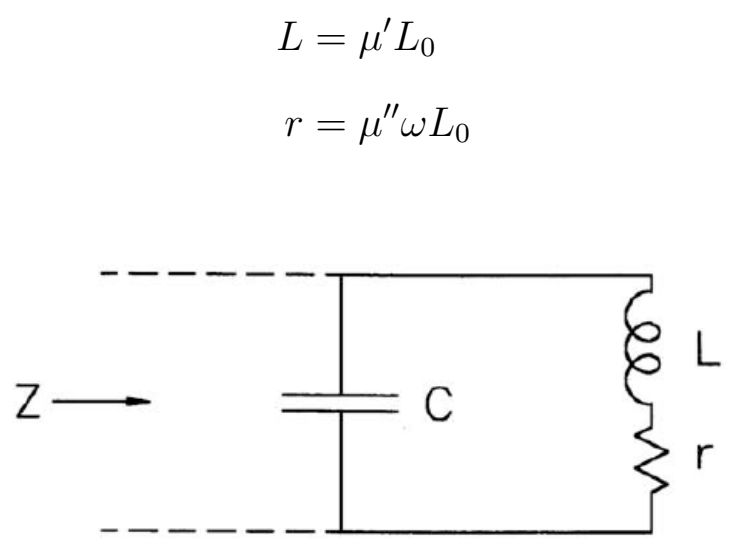

Figure 3.10: Parallel resonant circuit with ferrite-cored inductor

If this ferrite-loaded inductance is connected across a lossless capacitor as in Figure 3.10, then the impdacne of the circuit can be written as:

$$
\begin{aligned}
\frac{1}{Z} & =j \omega C+\frac{1}{r+j \omega L}=\frac{j \omega C r-\omega^{2} C L+1}{r+j \omega L} \\
Z & =\frac{r+j\left(\omega L-\omega^{3} C L^{2}-\omega C r^{2}\right)}{\left(1-\omega^{2} C L\right)}
\end{aligned}
$$


At resonance frequency:

$$
\begin{aligned}
& L=\omega_{0}^{2} C L^{2}+C r^{2} \\
& Z=r+\frac{\omega_{0}^{2} L^{2}}{r}
\end{aligned}
$$

If we define:

$$
Z=Q \omega_{0} L=\omega_{0} L\left(\frac{\omega_{0} L}{r}+\frac{r}{\omega_{0} L}\right)
$$

Thus the quality factor $Q$ can be written as:

$$
Q=\frac{\omega_{0} L}{r}+\frac{r}{\omega_{0} L}=\frac{\mu^{\prime}}{\mu^{\prime \prime}}+\frac{\mu^{\prime \prime}}{\mu^{\prime}}
$$

The dielectric loss in ferrite can be treated in the same manner as the magnetic loss. However, with NiZn ferrite, dielectric loss is not significant up to $30 \mathrm{MHz}$.

\subsubsection{The Co-axial Resonators}

For a ferrite-loaded co-axial cavity, the beam passes through the hollow central coaxial conductor and is accelerated by the electric field across the end capacitive gap. The circuit can be represented as Figure 3.11, where $C_{g}$ represents the gap capacitance:

The reactive impedance of such a resonator at the points $\mathrm{AB}$ is:

$$
Z=j Z_{0} \tan (\omega l / v)
$$

where $l$ is the length of the line, $v$ the velocity of the signal in the line and $Z_{0}$ the characteristic impedance of the line. Then:

$$
\begin{aligned}
& Z=j \omega L=j Z_{0} \tan (\omega l / v) \\
& L=\frac{Z_{0}}{\omega} \tan \frac{\omega l}{v}
\end{aligned}
$$




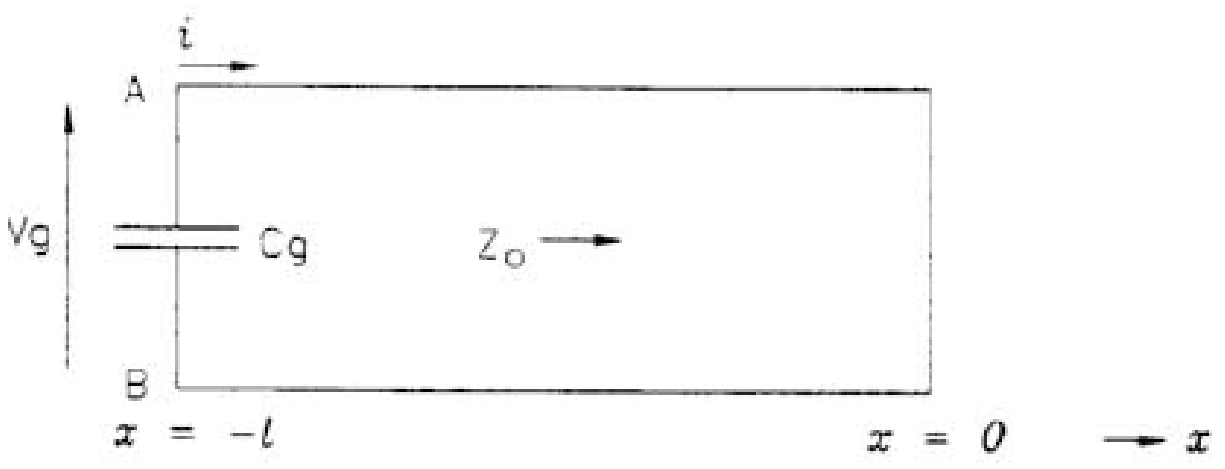

Figure 3.11: Equivalent circuit of co-axial resonator.

At resonance:

$$
\begin{aligned}
& \omega_{0}^{2}=\frac{1}{L C_{g}} \\
& l=\frac{v}{\omega_{0}} \tan ^{-1}\left(\frac{1}{Z_{0} \omega_{0} C_{g}}\right)
\end{aligned}
$$

A typical resonator structure is shown as in Figure 3.12. The capacitance of such a line in farads/meter is:

$$
C_{t}=\frac{2 \pi \epsilon_{e} \epsilon_{0}}{\ln \left(r_{3} / r_{1}\right)}
$$

and the inductance in henry/metre is:

$$
L_{t}=\frac{1}{2 \pi} \mu_{e} \mu_{0} \ln \left(r_{3} / r_{1}\right)
$$

with effective permittivity and permeability:

$$
\begin{aligned}
\epsilon_{e} & =\frac{\epsilon}{k+\epsilon(1-k)} \frac{d_{1}}{d_{1}+d_{2}} \\
\mu_{e} & =\left(1+k\left(\mu^{\prime}-1\right)\right) \frac{d_{1}}{d_{1}+d_{2}} \\
k & =\frac{\ln \left(r_{3} / r_{2}\right)}{\ln \left(r_{3} / r_{1}\right)}
\end{aligned}
$$



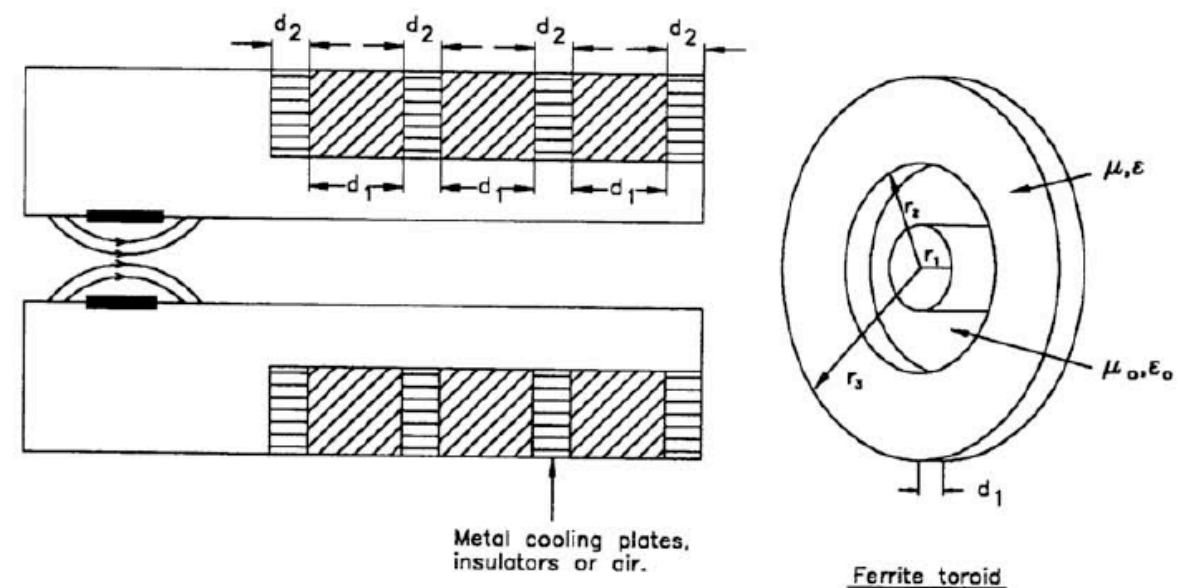

Ferrite toroid

Figure 3.12: Typical resonator structure

Thus the shunt impedance of the cavity can be expressed as:

$$
R_{s}=\omega_{0} Q L_{t}=A\left(\mu_{e} Q f\right)
$$

where $A=\mu_{0} \cdot l \cdot \ln \left(r_{3} / r_{1}\right)$, and exclusively depends on the geometry of co-axial resonator.

The wave velocity in such a line is:

$$
v=\frac{1}{\sqrt{L_{t} C_{t}}}=\frac{c}{\sqrt{\epsilon_{e} \mu_{e}}}
$$

where $c$ is the velocity of light. The characteristic impedance is:

$$
\begin{aligned}
Z_{0} & =\sqrt{\frac{L_{t}}{C_{t}}}=\frac{1}{2 \pi} \sqrt{\frac{\mu_{e} \mu_{0}}{\epsilon_{e} \epsilon_{0}}} \ln \frac{r_{3}}{r_{1}} \\
& =60 \sqrt{\frac{\mu_{e}}{\epsilon_{e}}} \ln \frac{r_{3}}{r_{1}}
\end{aligned}
$$

If the voltage at the capacitor is $V_{g} e^{j \omega t}$, the voltage and current are given by:

$$
\begin{aligned}
V & =-V_{g} e^{j \omega t} \frac{\sin \frac{\omega x}{v}}{\sin \frac{\omega l}{v}} \\
i & =j V_{g} e^{j \omega t} \frac{\cos \frac{\omega x}{v}}{Z_{0} \sin \frac{\omega l}{v}}
\end{aligned}
$$


We can see the current is maximum at the short circuit where $x=0$ and the ratio of the current at $x=-l$ to the current at $x=0$ is $\cos \frac{\omega l}{v}: 1$. This ratio determines the difference in the RF magnetic fields in the toroids at either end of the resonator.

The gap voltage can be estimated by the magnetic flux density in the cavity. From Faraday's Law:

$$
V_{g}=\left|\frac{d \Phi}{d t}\right|=\frac{d}{d t} \int_{r_{1}}^{r_{3}} B_{r f} \cdot l \cdot d r=B_{\max } \cdot \omega_{0} \cdot l \cdot r_{1} \cdot \ln \left(r_{3} / r_{1}\right)
$$

where the RF magnetic flux density $B_{R F}=B_{\max } \cdot r_{1} / r$ and $B_{\max }$ is the magnetic flux density around the inner conductor. Notice that here we have assumed $B_{r f}$ is a constant along the inner conductor.

\subsubsection{Transmission line Analysis on CIS Cavity}

Applying the above formulas in transmission line model, we carried out some analytical calculation for CIS cavity. The results are compared with SUPERFISH numerical simulation and experiment measurements.

The Poisson Superfish is a collection of programs developed and maintained by Los Alamos National Laboratory. The Poisson is for calculating static electric and magnetic filed, while Superfish is for the radio-frequency electromagnetic field. They can be used for both 2-D Cartesian coordinates or axially symmetric cylindrical coordinates. Figure 3.13 shows the structure of upper half cavity in SUPERFISH simulation.

For the CIS cavity, $r_{2}=r_{3}$, which gives $k=1$, so:

$$
\epsilon_{e}=\epsilon \frac{d_{1}}{d_{1}+d_{2}} \quad \mu_{e}=\mu^{\prime} \frac{d_{1}}{d_{1}+d_{2}}
$$

From measurement, $d_{1}=1$ inch, $d_{2}=0.5$ inch, so:

$$
\epsilon_{e}=\frac{2}{3} \epsilon \quad \mu_{e}=\frac{2}{3} \mu^{\prime}
$$




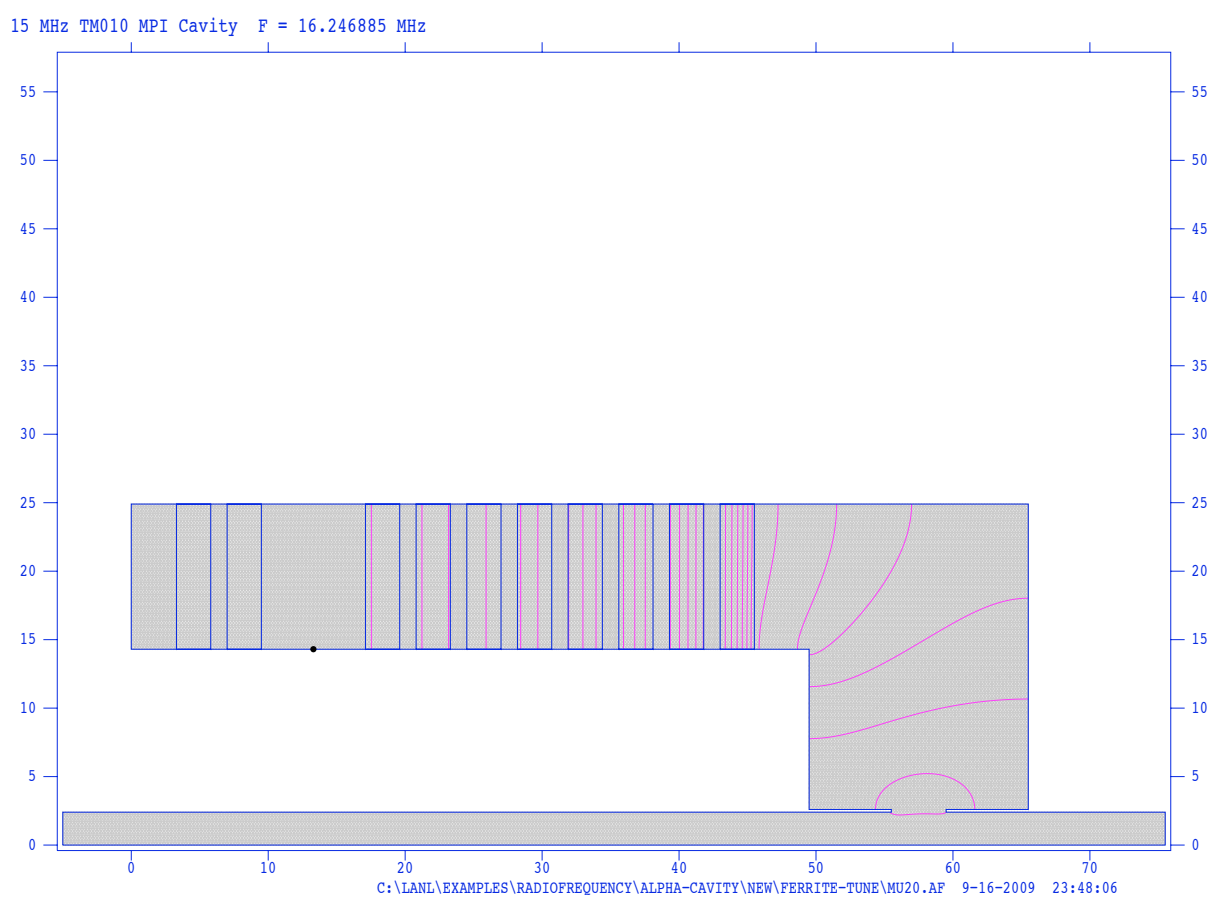

Figure 3.13: Upper half cavity structure in SUPERFISH simulation. The red lines are the electric field lines. 
Also from measurement, the inner and outer radius of the cavity:

$$
r_{1}=14.3 \mathrm{~cm} \quad r_{2}=24.9 \mathrm{~cm}
$$

So the characteristic impedance:

$$
Z_{0}=60 \sqrt{\frac{\mu_{e}}{\epsilon_{e}}} \ln \frac{24.9}{14.3}=33.28 \sqrt{\frac{\mu^{\prime}}{\epsilon}}
$$

The effective length of the cavity can be expressed as:

$$
l=\frac{3 c}{2 \sqrt{\epsilon \mu^{\prime}} \omega_{0}} \tan ^{-1} \frac{1}{33.28 \sqrt{\frac{\mu^{\prime}}{\epsilon}} \omega_{0} C_{g}}
$$

From this expression, we can do the following analysis:

\section{The frequency tuning by ferrite effective permeability $\mu$}

The transmission line theory shows that for given $l, C_{g}$ and $\epsilon, \sqrt{\mu^{\prime}} \omega_{0}$ is fixed. Thus we can tune the cavity frequency by changing $\mu^{\prime}$. The resonant frequency $\omega_{0}$ should be inverse proportional to $\sqrt{\mu^{\prime}}$ :

$$
\omega_{0} \propto 1 / \sqrt{\mu^{\prime}}
$$

Or:

$$
\log (\mathrm{f} 0)=\frac{1}{2} \log \left(\mu^{\prime}\right)+\text { constant }
$$

We simulate the tuning process in SUPERFISH and plot the linear fitting between $\log (\mathrm{f} 0)$ and $\log \left(\mu^{\prime}\right)$. The result is shown in Figure 3.14. The clear linear relation and the slope of 0.5 verify the conclusion from transmission line theory.

\section{Effective length of cavity}

From the previous measurement on CIS cavity, we estimated that the gap capacitance $C_{g} \approx 60 \mathrm{pF}$. In the SUPERFISH code, we used relative permittivity $\epsilon=14.5$. With 


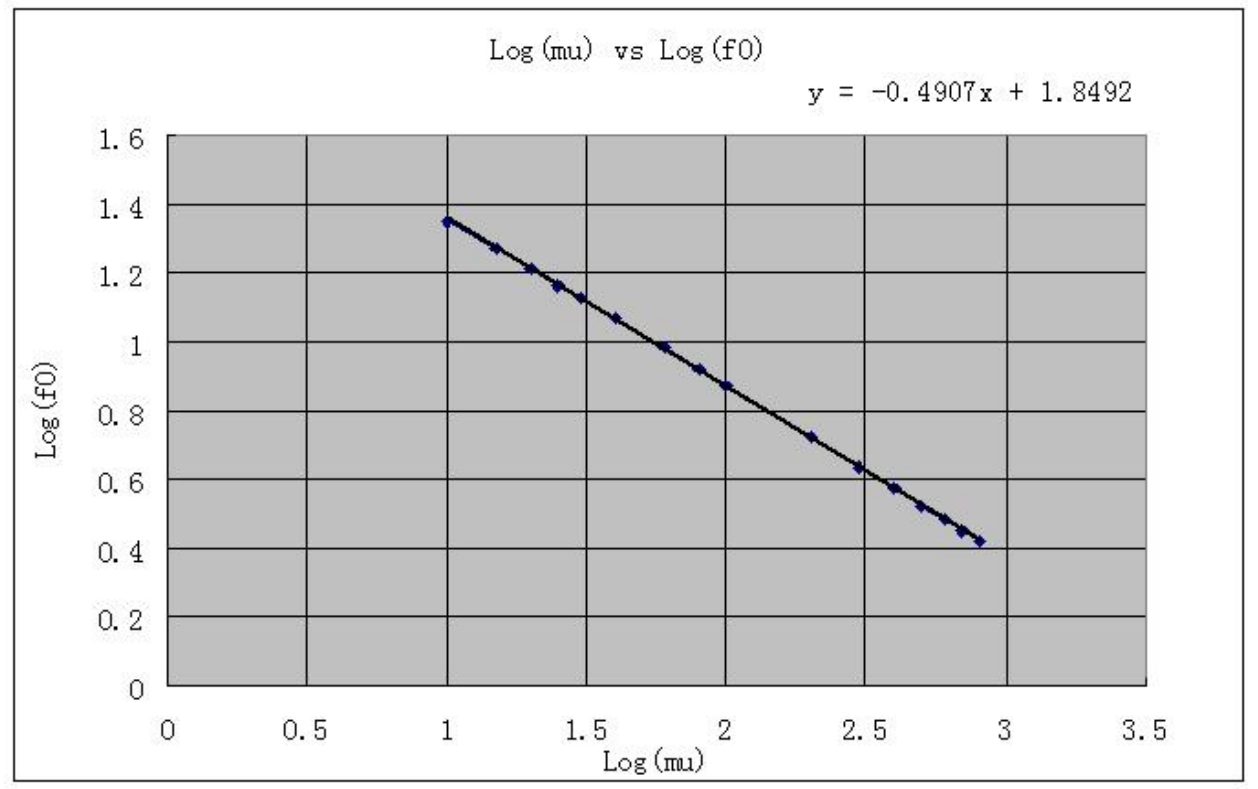

Figure 3.14: Changing the relative permeability in SUPERFISH to tune the resonant frequency. By linear-fitting $\log \left(\mu^{\prime}\right)$ vs $\log (f 0)$, we can see a very clear $\omega_{0} \propto 1 / \sqrt{\mu^{\prime}}$ relation 
the permeability and corresponding resonant frequency calculated by SUPERFISH, we can calculate the effective length at different resonant frequencies by:

$$
l=\frac{3 c}{2 \sqrt{\epsilon \mu^{\prime}} \omega_{0}} \tan ^{-1} \frac{1}{33.28 \sqrt{\frac{\mu^{\prime}}{\epsilon}} \omega_{0} C_{g}}
$$

This is the real physical length of the cavity, so it should not depend on the resonant frequency. By changing the permeability $\mu$ in the SUPERFISH simulation, we can calculate the effective length of cavity at different resonant frequencies. The calculated result is shown in Figure 3.15.

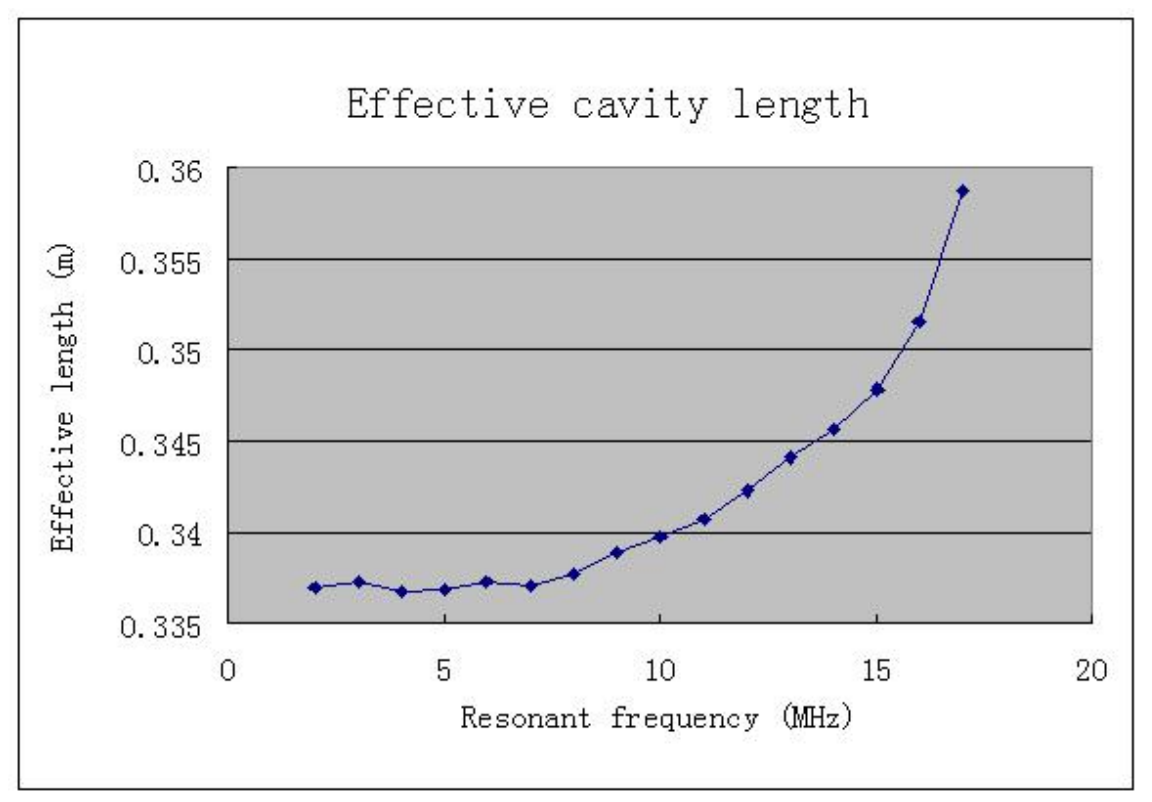

Figure 3.15: The effective length calculated by combining the simulation result of SUPERFISH and the formula from transmission line model.

We can see from $2 \mathrm{MHz}$ to $17 \mathrm{MHz}$ range, the results are within $0.34 \mathrm{~m}$ to $0.36 \mathrm{~m}$. The real length is:

$$
l_{\text {real }} \approx(2.5+1.2) \times 10=37 \mathrm{~cm}
$$


So they agree with each other within $10 \%$.

\section{Phase advance in the cavity}

The phase advance in the cavity is:

$$
\frac{\omega_{0} l}{v}=\tan ^{-1} \frac{\sqrt{\epsilon}}{33.28 \sqrt{\mu^{\prime} \omega_{0} C_{g}}}
$$

If $l, C_{g}$ and $\epsilon$ are fixed, then $\sqrt{\mu^{\prime}} \omega_{0}$ is also fixed, so the phase advance in the cavity is also fixed. It doesn't change when we tune the resonance frequency by changing the bias field.

For CIS:

$$
\frac{\omega_{0} l}{v} \approx 1.33=0.42 \pi \approx 0.5 \pi
$$

It is almost a quarter-wave cavity. From the expression of phase advance, we can also see that the smaller the gap capacitance is, the closer it resembles a "quarter-wave".

\subsection{High Power Test of shortened CIS RF Cavity}

\subsubsection{Shortened CIS RF Cavity}

Due to the limited space, we need to shorten the length of the CIS cavity to fit it into ALPHA storage ring. After the simulation in SUPERFISH, we decide to remove 3 ferrite disks under the main bias loop. The whole cavity is shorten by 4.5 inch.

In order to shorten the cavity, we need to open the original cavity, disassemble it, remove the ferrite disks, cut the bias quadrupole magnet and assemble everything back. During this process, we have learnt more detailed knowledge of such kind of cavity and got some inspiration for designing a new similar cavity later. 


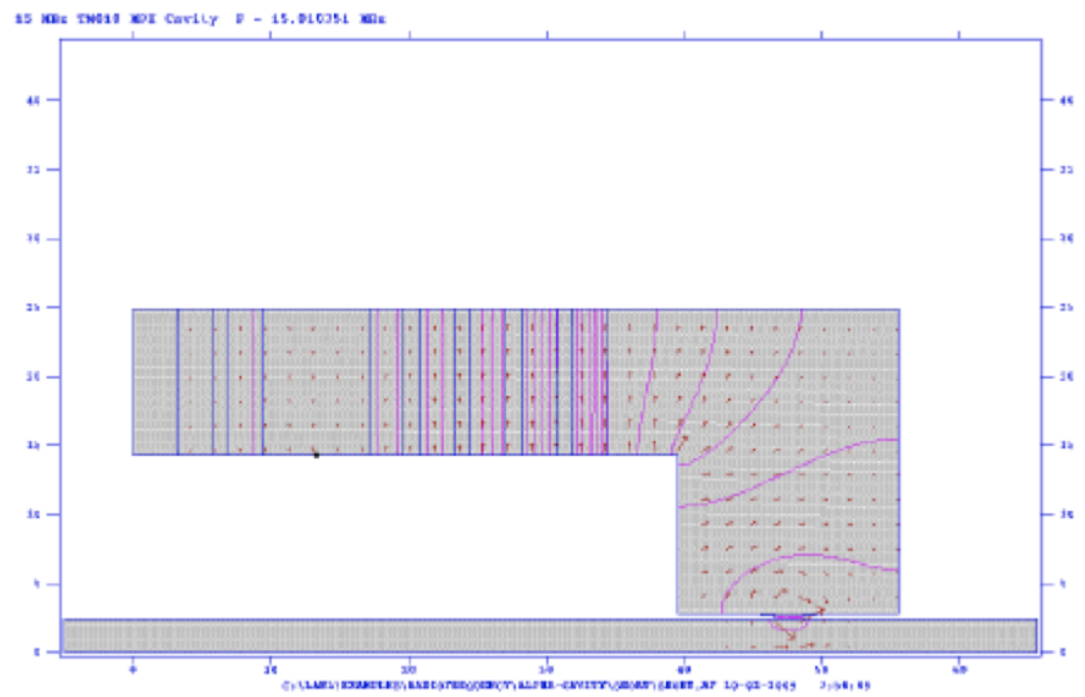

Figure 3.16: The SUPERFISH simulation of the shortened CIS RF cavity 


\subsubsection{High Power Test}

After reducing the cavity length, we did the high power test to measure the accelerating voltage and the shunt impedance.

The cavity is powered by an A300 solid state amplifier, which is the power source for the original CIS cavity. It can deliver up to 300 watts and withstand $100 \%$ power reflection.

The high power test result is shown in Figure 3.17 and the corresponding shunt impedance calculation is shown in Figure 3.18.

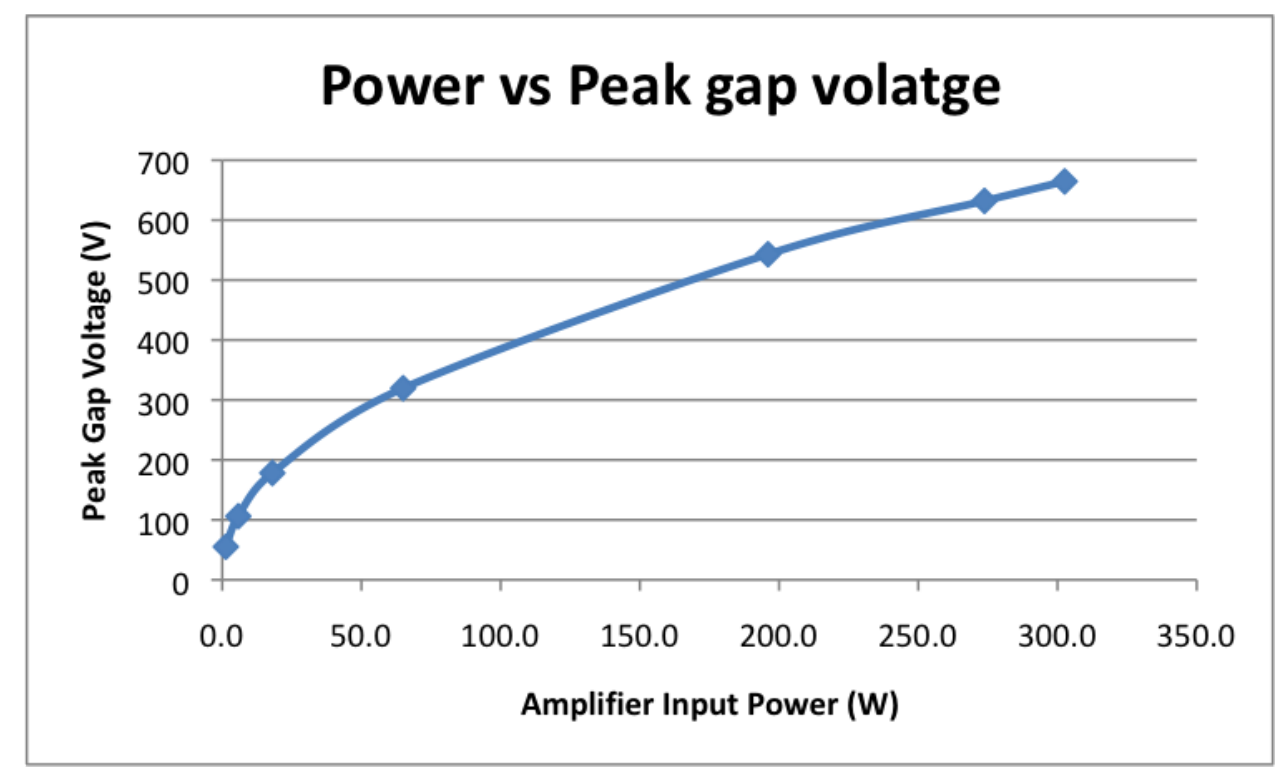

Figure 3.17: The High Power Test of the Reduced RF Cavity: The Accelerating Voltage

The results show that in the low power range, the shunt impedance has improved from a few hundred ohm to more than $1000 \mathrm{ohm}$. Thus by reducing the cavity length and optimizing both bias current and external capacitor, the efficiency of the cavity has been improved a lot. When the input power goes up to $300 \mathrm{~W}$, which is the 


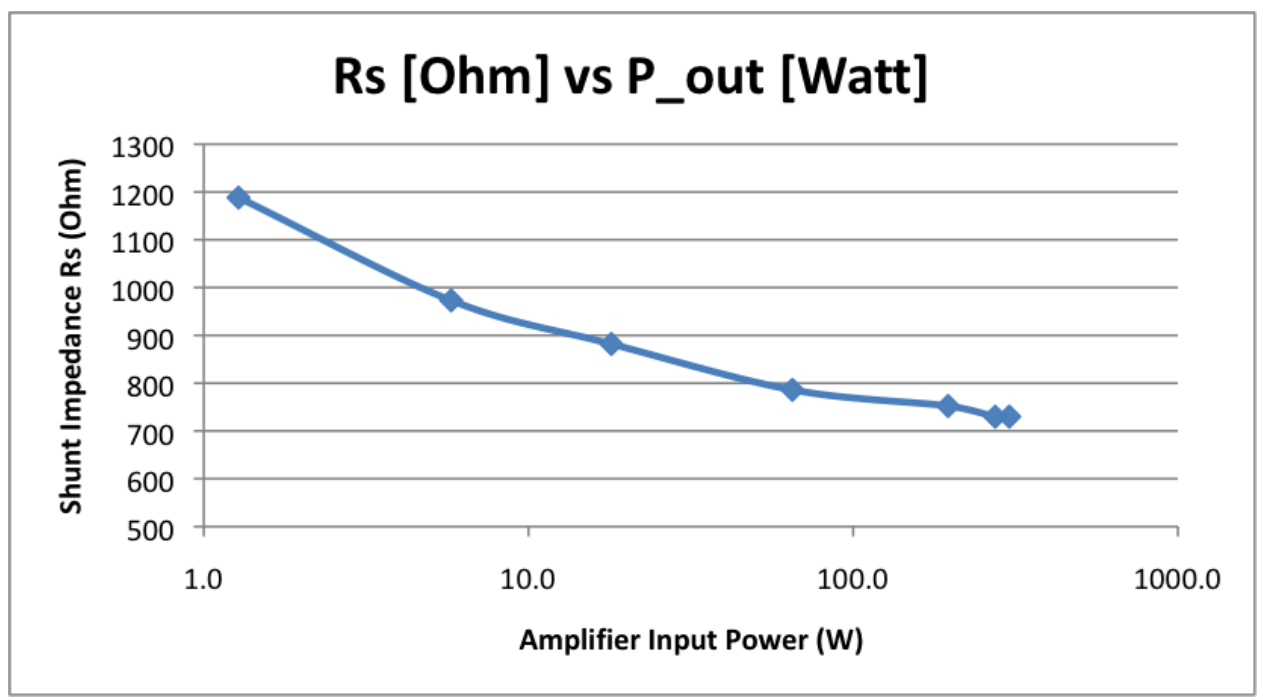

Figure 3.18: The High Power Test of the Reduced RF Cavity: The Shunt Impedance

maximum output of the A300 Solid Amplifier, the peak voltage across the ceramic gap achieves about 700 volts. This is still far from $3 \mathrm{KV}$, which is our desired level of the RF Cavity voltage. We also notice that as the input power increases, the shunt impedance of the cavity decreases. This is due to the decrease of the $\mu Q f$ value of the ferrite material. As the power increases more, we expect the shunt impedance will drop even more.

\subsection{The Performance Limit of CIS RF Cavity}

In the high power test of the reduced CIS RF Cavity, we have achieved about 700 volts with $300 \mathrm{~W}$ input power. It seems that to achieve higher accelerating voltage, we only need to increase the input power. However, as we have seen, the shunt impedance keeps on decreasing as the input power increases, which indicates we might need up to 
$1 \mathrm{~kW}$ to achieve $1 \mathrm{KV}$ accelerating voltage, and $10 \mathrm{~kW}$ for $3 \mathrm{kV}$ cavity voltage. When the power goes up, the cooling will also become an issue. A more serious concern is the intrinsic performance limitation of the ferrite material in the cavity, which may start to behave nonlinearly above certain power threshold. This nonlinear behavior is called High Loss Effect (HLE). It imposes the limit on the accelerating voltage for a ferrite loaded cavity. Since ALPHA cavity has a much higher voltage requirement than CIS cavity, we need to examine the HLE threshold of ALPHA cavity operation.

\subsubsection{The High Loss Effect}

In the ferrite-loaded cavity, the maximum usable accelerating voltage is often strongly restricted by the High Loss Effect (HLE). When the cavity is operated above the HLE threshold, the accelerating voltage will follow the input power pulse only for a short time and then drop to a lower level with noisy amplitude. Any further increase of the voltage requires disproportionately more input power. Figure 3.19 shows an example of HLE [16].

The previous study on the HLE [17] shows that the threshold value is apparently related to the stored RF energy in the ferrite. The mechanism of HLE appears to be related to a large scale coherent oscillation within the ferrite, which can be attenuated or destroyed by the frequency modulation of the exciting RF field.

\subsubsection{The limit of the ferrite of CIS RF Cavity}

The ferrite material used in CIS RF Cavity is Philips 8C12. It has a large permeability tuning range by adjusting the bias magnetic field across the ferrite, thus very suitable for the frequency ramping up from $2 \mathrm{MHz}$ to $10 \mathrm{MHz}$ during the acceleration in CIS. It has been used in many ferrite loaded cavities.

After some survey, we found information on Philips 8C12's HLE threshold mea- 

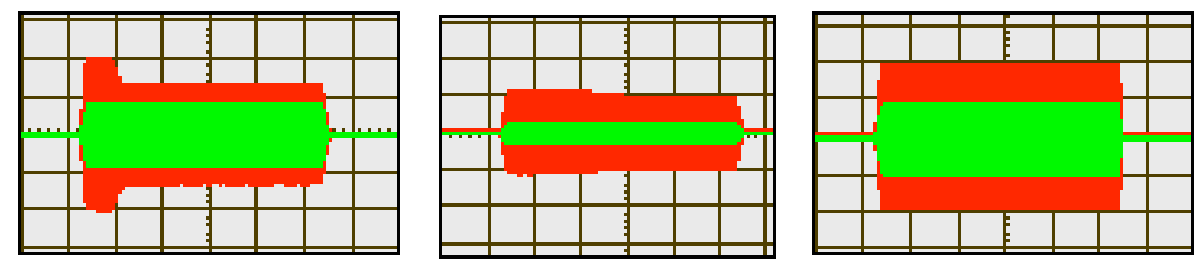

Figure 3.19: The Example of High Loss Effect [16]. The green line is the input power and the red line is the accelerating voltage. The left figure shows the severe HLE when the cavity is operated way above the HLE threshold. The middle figure shows the mild HLE when it's slightly above the threshold. The right figure is the normal cavity operating condition without HLE.

surement in [16], as shown in Figure 3.20. Using the transmission line theory, we can estimate the maximum RF magnetic field in the cavity is about $17 \mathrm{mT}$ to establish 1 $\mathrm{KV}$ accelerating voltage. To tune the resonant frequency of the reduced CIS cavity, we have applied $20 \mathrm{Amp}$ and 15 Amp to the main bias current and loop bias current respectively. Considering the 50 turns of the bias current, the effective DC bias is larger than $750 \mathrm{~A} / \mathrm{m}$. From Figure 3.20, we can deduce that $17 \mathrm{mT}$ will be above the HLE threshold for the $750 \mathrm{~A} / \mathrm{m}$ DC bias.

After realizing this accelerating voltage limit imposed by the HLE of $8 \mathrm{C} 12$ ferrite material, we have to give up the plan of refurbishing CIS cavity for the ALPHA use. Instead, we need to find a more suitable ferrite material and redesign the cavity.

\subsubsection{A more suitable ferrite material}

The new ferrite should have the following properties: 


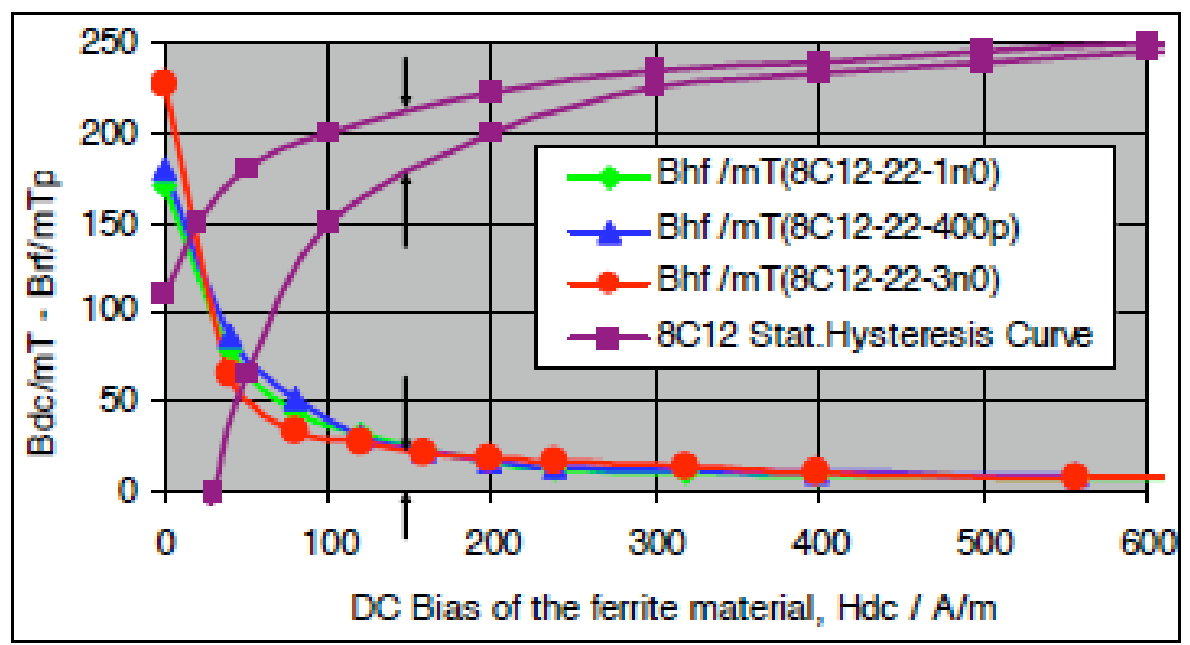

Figure 3.20: The High Loss Effect threshold measurement of Philips 8C12 [16]

1. The permeability range should match the requirement of the operating frequency. A suitable permeability can also simplify the frequency tuning scheme of the cavity.

2. The loss of a heavy ferrite-loaded cavity is mainly from ferrite loss. The shunt impedance of such a cavity is proportional to $\mu Q f$, which is a character of ferrite itself. To achieve higher efficiency and reduce heat dissipation, we want a ferrite with large $\mu Q f$.

3. The RF magnetic field corresponding to the maximum accelerating voltage should be below the HLE threshold of this ferrite material.

After searching for a while, we have decided on Toshiba M4C21A. We will show the design of the new cavity with this ferrite disk in the next section and how the performance of the cavity has been improved. 


\subsection{Design of the New RF Cavity}

With the new ferrite disk, we will build a new RF Cavity for ALPHA. The parameters of the new ferrite disk have been measured in [18]:

Table 3.1: Parameters of M4C21A ferrite torus

\begin{tabular}{|c|c|c|c|c|}
\hline Inner Diameter & Outer Diameter & Thickness & Permeability & Permittivity \\
\hline 5 inch & 8 inch & 1 inch & $54 \mu_{0}$ & $13 \epsilon_{0}$ \\
\hline
\end{tabular}

\subsubsection{The SUPERFISH Design of the New RF Cavity}

When designing the new RF cavity, we have followed the following guidelines:

1. Due to the space limit in the ALPHA storage ring, the length of the cavity should be compact.

2. Space is needed between each ferrite for air cooling.

3. The resonant frequency is fixed at around $15 \mathrm{MHz}$. There should also be a frequency tuning component to finely tune the resonant frequency.

4. The impedance should be matched between the input power cable and the cavity to get an efficient power transmission.

We have designed the new cavity in SUPERFISH, shown in Figure 3.21. The magnetic field distribution along the inner conductor is shown in Figure 3.22.

For the new design, we choose the co-axial quarter wave structure similar to CIS cavity. There are total 6 ferrite disks in the cavity, thus the cavity length has been 


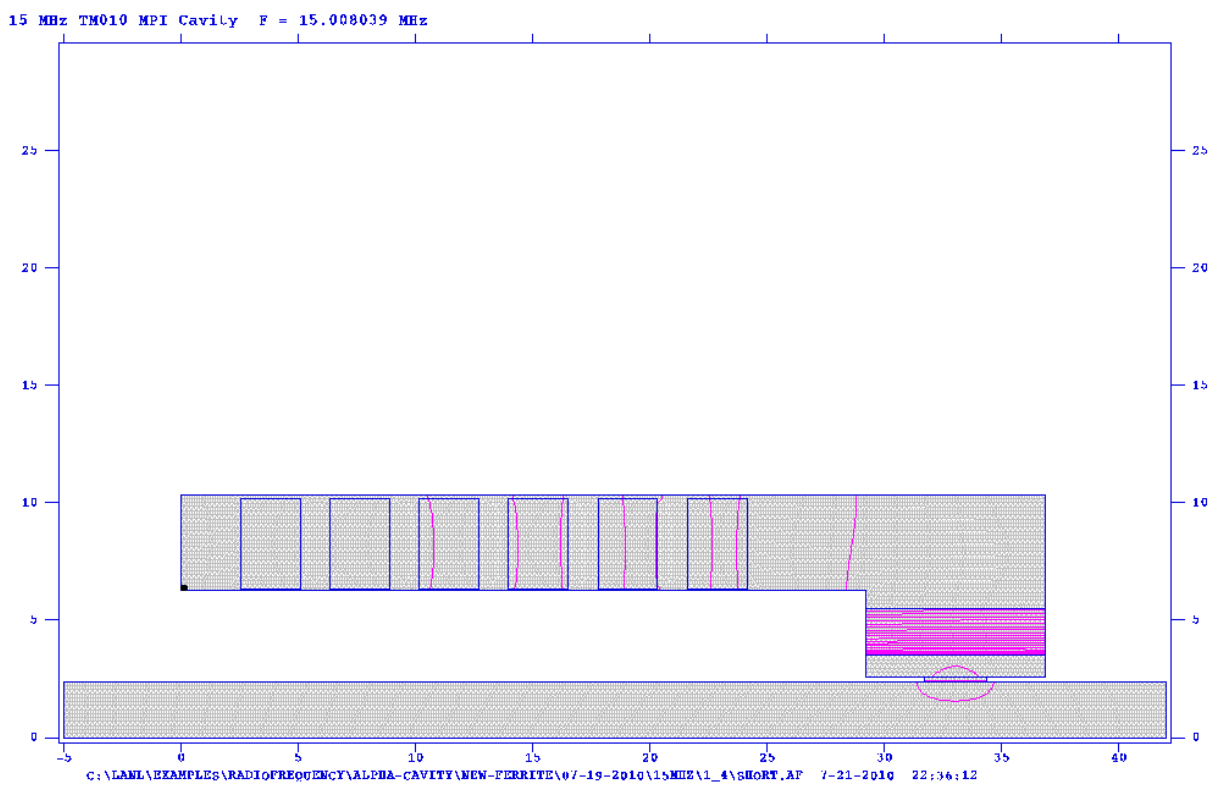

Figure 3.21: The SUPERFISH design of the new ferrite cavity

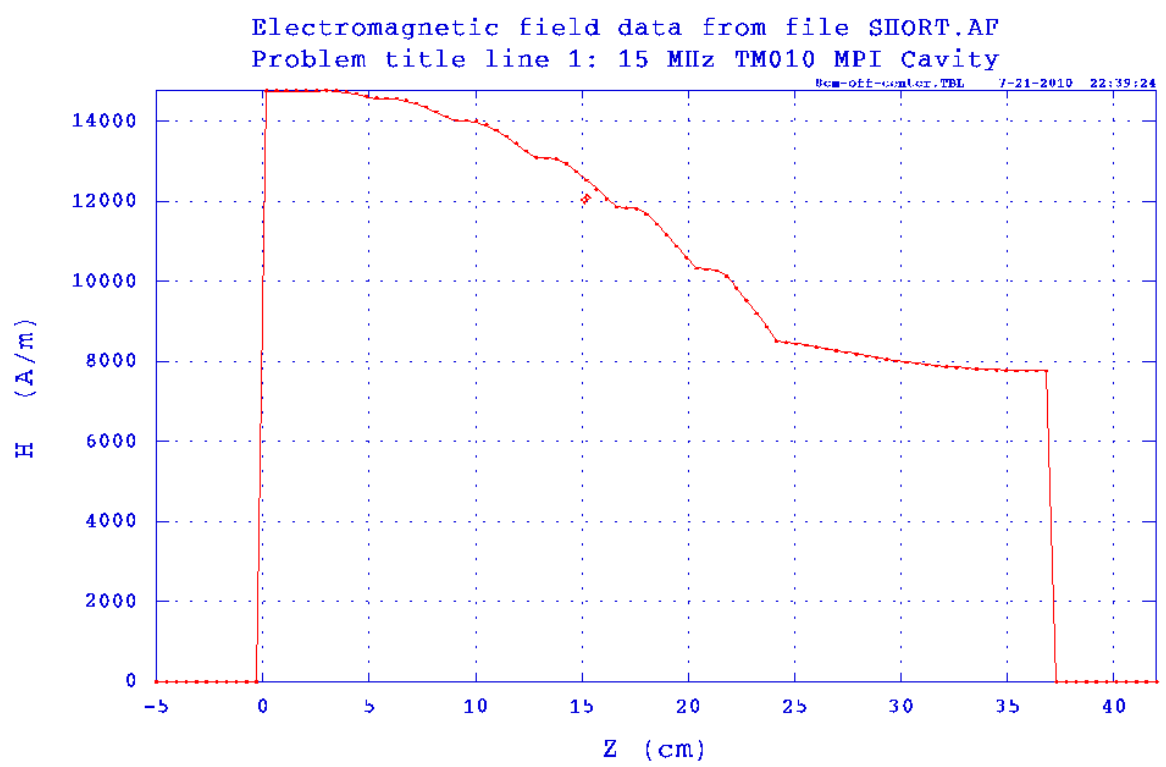

Figure 3.22: The magnetic field distribution of 1st harmonic mode. 
reduced to less than $40 \mathrm{~cm}$. An external capacitor is installed across the ceramic gap to adjust the resonant frequency. The external quadrupole iron is not needed anymore. The impedance matching can be done by an external impedance adapter at the power input port.

Besides the $15 \mathrm{MHz}$ fundamental mode, we also study the higher order modes of this design. The SUPERFISH frequency scan shows a 2nd mode frequency at 53 $\mathrm{MHz}$ and 3rd mode at $97 \mathrm{MHz}$, shown in Figure 3.23. The magnetic field distribution along the inner conductor of both modes are shown in Figure 3.24 and Figure 3.25.

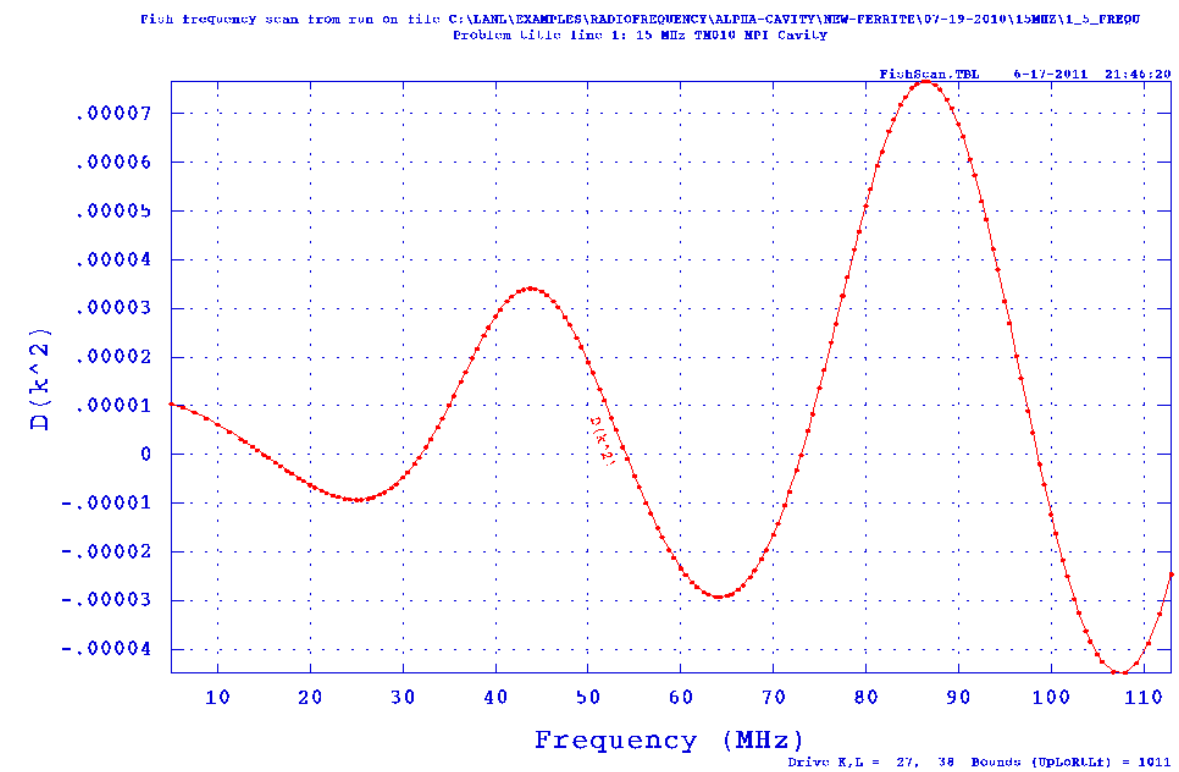

Figure 3.23: The resonant frequency scan by SUPERFISH. When $D\left(k^{2}\right)=0$ and its slope is negative, the corresponding frequency is the resonant frequency.

Compared with the CIS cavity, the new cavity design has been improved in the following aspects: 


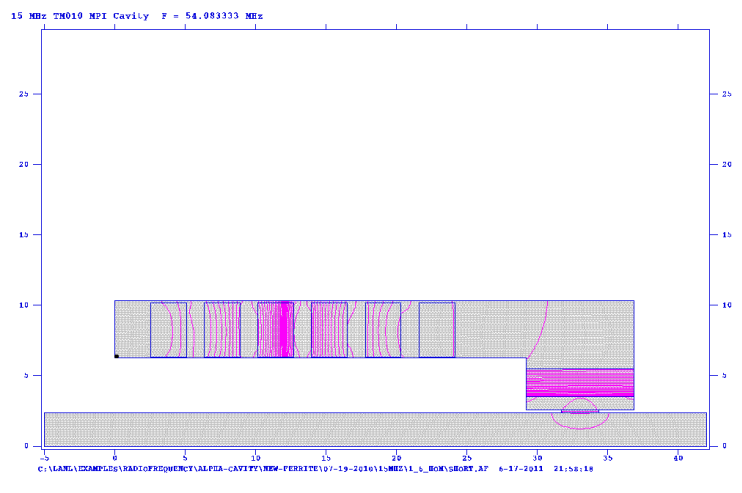

(a)

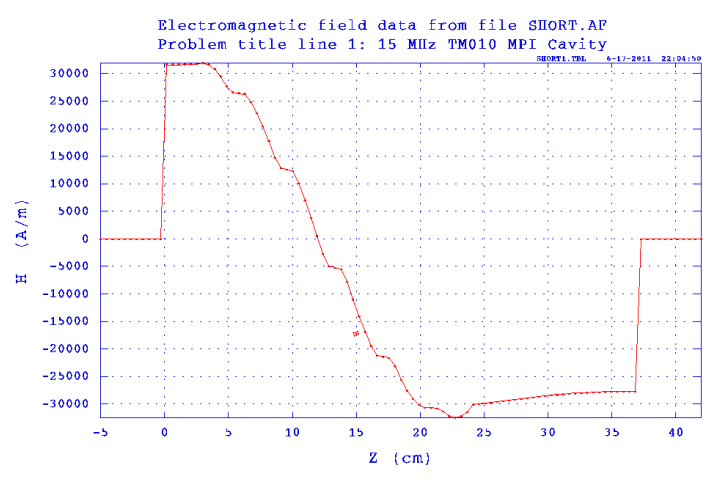

(b)

Figure 3.24: Magnetic field distribution of 2nd harmonic mode.

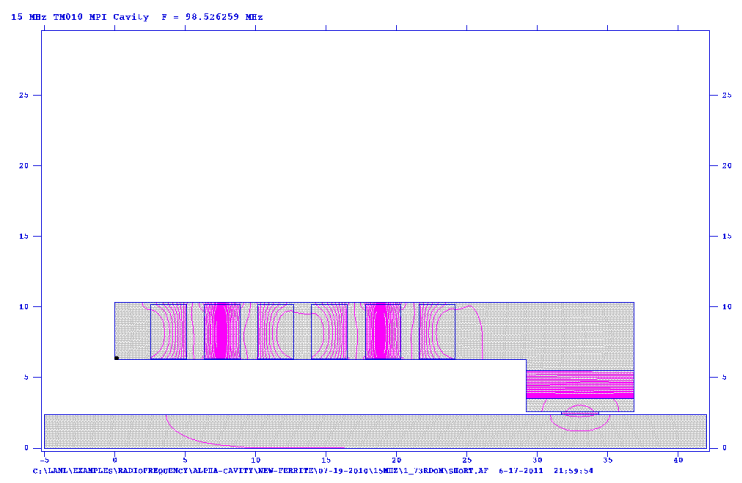

(a)

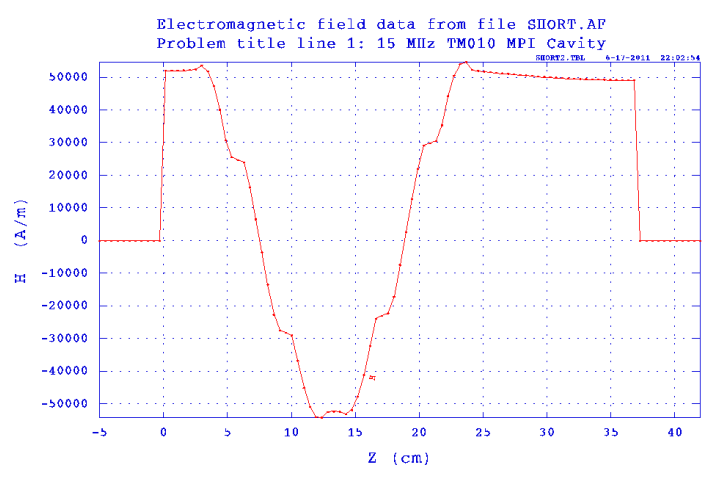

(b)

Figure 3.25: Magnetic field distribution of 3rd harmonic mode. 
1. Due to the suitable value of M4C21A permeability and permittivity, the total length of cavity has been reduced a lot. The frequency tuning is also much easier. Since the cavity will be operated under a fixed frequency, no external quadrupole magnet is needed anymore. All the frequency tuning is done by the external capacitor.

2. The $\mathrm{Q}$ value of $\mathrm{M} 4 \mathrm{C} 21 \mathrm{~A}$ at $30 \mathrm{MHz}$ is about 200 . The $\mu Q f$ factor is estimated to be about 20 times larger than $8 \mathrm{C} 12$, so the shunt impedance will also be much larger. The cavity efficiency will be much higher.

3. The HLE threshold of M4C21A has been tested by [17], shown as the "type C" curve in Figure 3.26. By the transmission line analysis and SUPERFISH simulation of the new design, we can estimate that the root mean square (rms) of RF Flux when the cavity is operated at $3 \mathrm{KV}$ is about 50 Gauss. If we extrapolate the data in Figure 3.26 to the zero bias magnetic field, we can see the HLE threshold of this material is larger than 50 Gauss. Thus we can safely go up to $3 \mathrm{KV}$ accelerating voltage without tripped by HLE. 


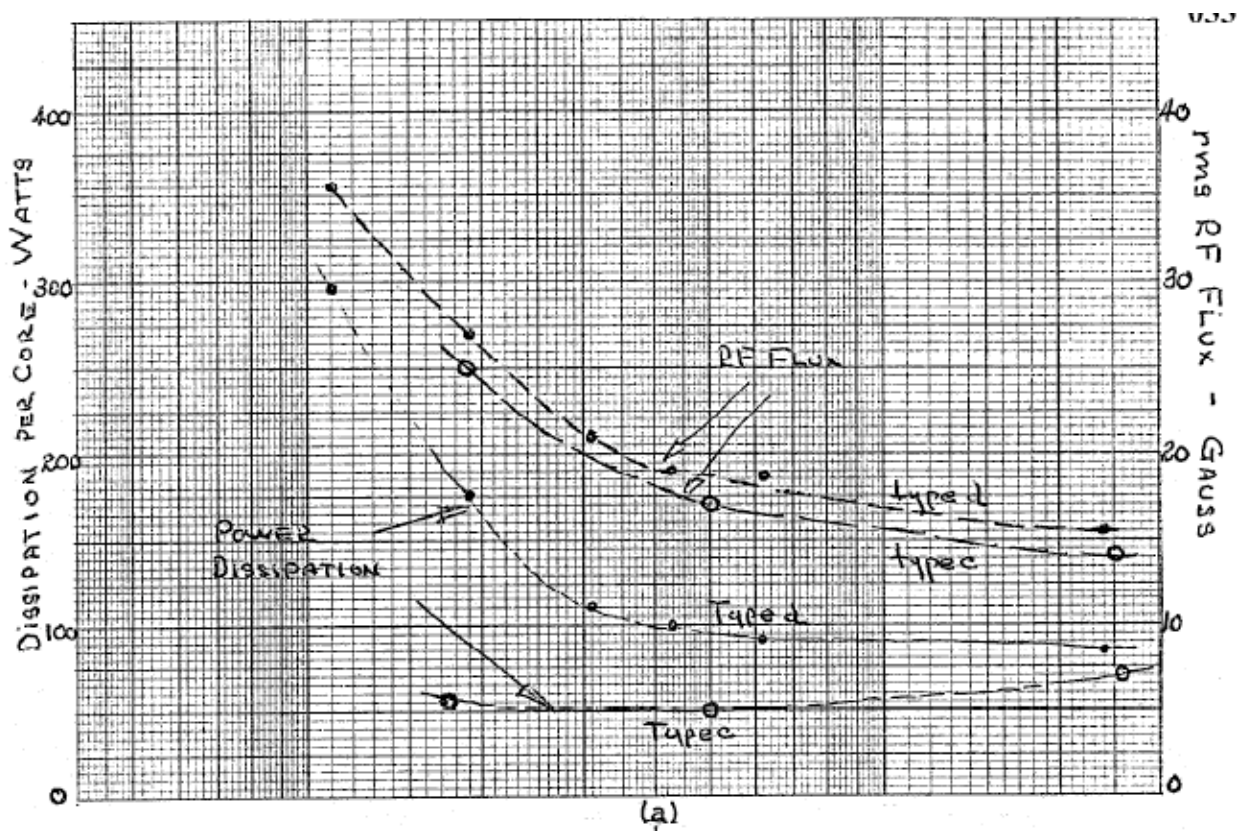

Figure 3.26: The HLE Threshold Measurement of M4C12A [17] 


\section{Chapter 4}

\section{Stripline Fast Extraction Kicker}

One of the primary requirements for the radiation effects testing program is the intensity and uniformity of the electron radiation dose. During the accumulation operating mode, we will accumulate up to $1000 \mathrm{nC}$ of electron beam with a length of $50 \mathrm{~ns}$ and kick them to the extraction line in one pulse. In this chapter, we present the design of this fast extraction kicker.

\subsection{Specification of the Kicker}

The circumference of the ALPHA ring is $20 \mathrm{~m}$ (66.7 ns). For the beam length of 50 ns, the time window for the kicker to establish the kicking field should be no more than $10 \mathrm{~ns}$. The length of kicker is limited by the available space for the kicker in the ring, which is confined to be $450 \mathrm{~mm}$ from flange to flange. The short length of the kicking field imposes a high voltage requirement on the kicker. The specification [19] of the kicker is shown in Table 4.1.

Considering the fast rising time and high voltage requirement, we decided on a traveling wave kicker with stripline structure. 
Table 4.1: Kicker Specification.

\begin{tabular}{l|c}
\hline Beam Energy $E_{b}$ & $50 \mathrm{MeV}$ \\
Kicker Gap $h$ & $47.6 \mathrm{~mm}$ \\
Kicker Vacuum Champer ID & $73.0 \mathrm{~mm}$ \\
Field Rise Time & $10 \mathrm{~ns}$ or less \\
Maximum/minimum Kick Angle $\theta$ & $23 / 10 \mathrm{mrad}$ \\
Duration of Kicker & $100 \mathrm{~ns}$ \\
Flange to flange distance & $45 \pm 0.2 \mathrm{~cm}$ \\
Length of High field region & $30-35 \mathrm{~cm}$ \\
\hline
\end{tabular}

The kicker gap is chosen to be the same as the inner diameter (ID) of the beam pipe to reduce the discontinuity and wakefield. The strip length is chosen to be $30 \mathrm{~cm}$. The ID of kicker vacuum chamber is determined by the available vacuum pipes and conical reducers from commercial suppliers.

The stripline kicker has a similar structure as a stripline beam position monitor (BPM), but is operated in the opposite way, as show in Figure 4.1. The left figure shows the general structure of the kicker. The pulsed power is fed into the downstream port, travels to the upstream port, and dissipated on the dummy load. An electromagnetic flux will travel between the strips with the pulsed power. The two strips have opposite electric polarity to double the voltage across the strips. The right figure shows the force that a charged particle undergoes when traveling through the electric-magnetic flux. If the particle travels in the same direction of the E-M field 
flux, labeled as direction "1", the electric field and the magnetic field will cancel each other and the net force is zero. In this case, there isn't any deflecting effect. If the particle travels in the opposite direction to the E-M field flux, labeled as direction "2", the electric and magnetic force are in the same direction and the total deflecting effect has been doubled. This is why we feed the power from the downstream port to the upstream port. Comparing with the electro-static kicker, the traveling wave kicker has much lower efficiency, because a lot energy will be dumped to the dummy load. The advantage is that the kicking field has been doubled.
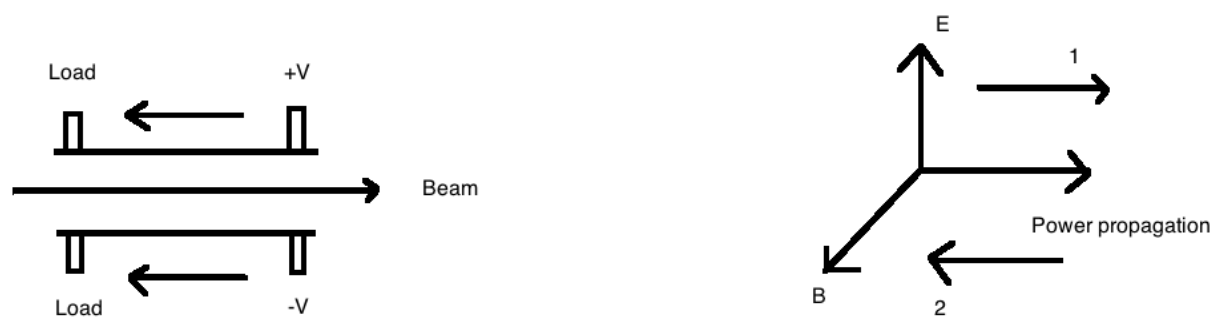

Figure 4.1: The general structure and operating principle of the stripline traveling wave kicker.

\subsection{Numerical Simulation}

The simulation of the kicker design are done with the 2D code Poisson [20] and 3D code Microwave Studio [21]. In the 2D Poisson simulation, we study different geometries of the stripline and choose the one that gives us a uniform kicking field. From this geometry we start the 3D study in Microwave Studio. The strip is trimmed 
to match $50 \Omega$ characteristic impedance. Energy transmission and time response are also investigated.

\subsubsection{D Poisson Simulation}

Using the 2D Poisson simulation, we have studied the effects of the geometry of the electrode on the uniformity of electric field across the strip, especially on the beam path. Figure 4.2 shows an example of $2 \mathrm{D}$ electric field and Figure 4.3 shows the corresponding kicking field vs transverse position $x$.

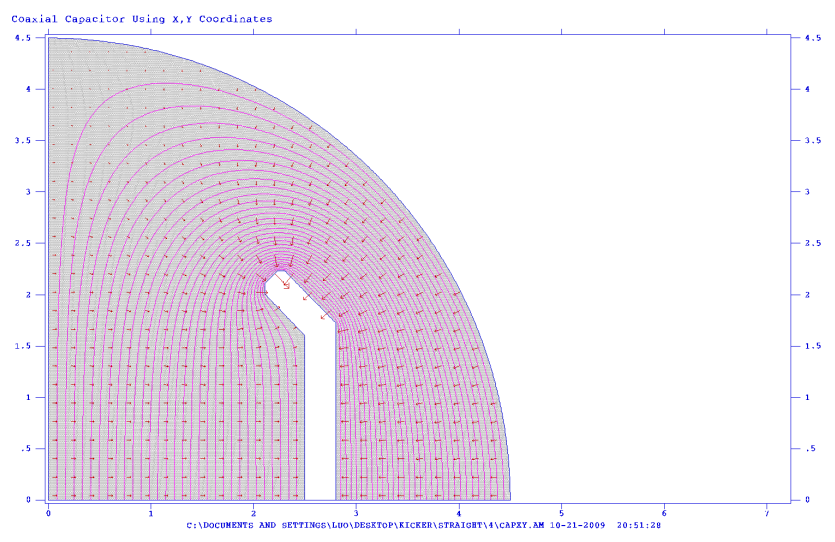

Figure 4.2: $2 \mathrm{D}$ electric field line in a quarter of kicker cross section.

The distance between the electrodes is fixed to be the same with beam pipe diameter. The tips of the electrode are bent inwards to expand the uniform kicking field region in the center. In Poisson simulation, we have trimmed the electrode tips with different bending angles and lengths. The geometry that gives the best uniformity of kicking field is chosen as the start point for the 3D Microwave Studio design. 


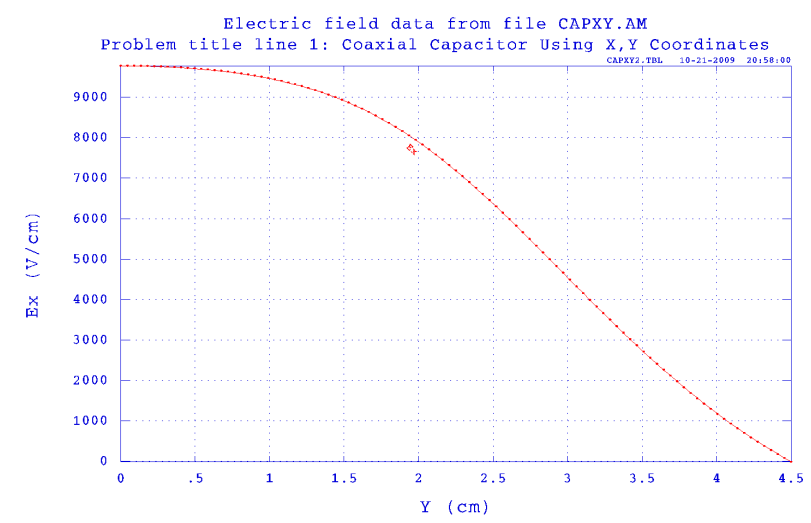

Figure 4.3: Kicking field along the central transverse line parallel to stripline.

\subsubsection{D Microwave Studio Simulation}

CST Microwave Studio is a powerful commercial software for the 3D electromagnetic field simulation at high frequency. It is widely used in accelerator science and other fields for radio frequency $(\mathrm{RF})$ simulation. By Microwave Studio, we have refined the kicker electrodes to match a $50 \Omega$ characteristic impedance, checked the uniformity of electric field and calculated the real time response of the power pulse.

\section{Impedance Matching}

The electric power is fed into the kicker by the coaxial cable, which often has a characteristic impedance of $50 \Omega$. To get the maximum power transmission, the characteristic impedance of the kicker should be $50 \Omega$, which is identical to the impedance of the coaxial power cable. For the kicker operated under high power pulse, the impedance matching is particularly important for the protection of the power source. Another advantage is that the good matching can reduce the power ripples and fasten the process of establishing a stable kicking field. 
For a transmission line structure composed of $\mathrm{N}$ conductors, it has N-1 orthogonal TEM modes. Each mode has its own electromagnetic field pattern and its own characteristic impedance. For example, the coaxial cable has inner and outer conductor, so it has only one TEM mode and one characteristic impedance. The stripline kicker we design has 3 conductors, including two electrodes and the vacuum chamber. Thus it has two TEM modes and two characteristic impedances, as shown in Figure 4.4. The mode in which the two electrodes have the same charge is called even TEM mode. It corresponds to the image charge induced by the beam passing through the center of the kicker. The mode in which the two electrodes carry opposite charge is called odd TEM mode, which corresponds to the kicking field. This is the mode that we should consider for matching.
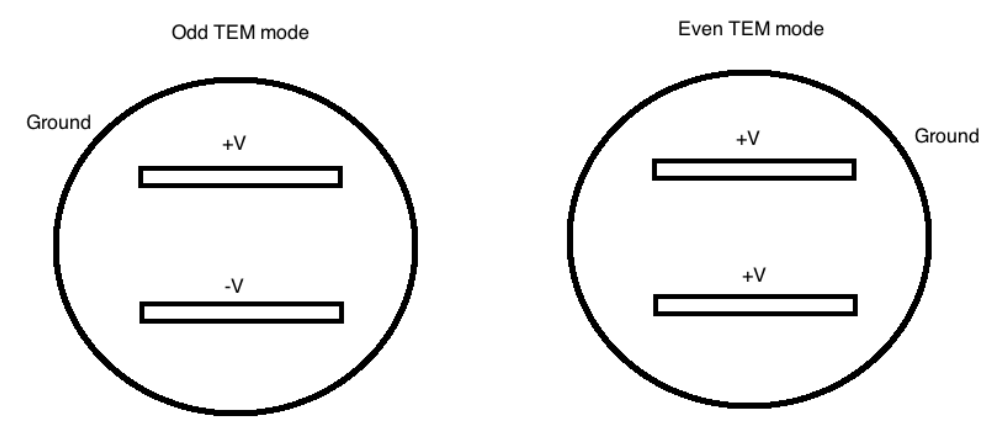

Figure 4.4: The odd TEM mode and the even TEM mode of the kicker.

After determining a rough geometry from the Possion simulation, we construct the kicker in the Microwave Studio and trim the strip electrode to get a $50 \Omega$ characteristic impedance for odd TEM mode. Figure 4.5 shows the optimized design and odd TEM mode. In the simulation, we construct only the upper half of the kicker and impose proper boundary condition: the electric field is defined to be perpendicular 
everywhere on the central plane of the kicker. This scheme reduces the calculation burden significantly.

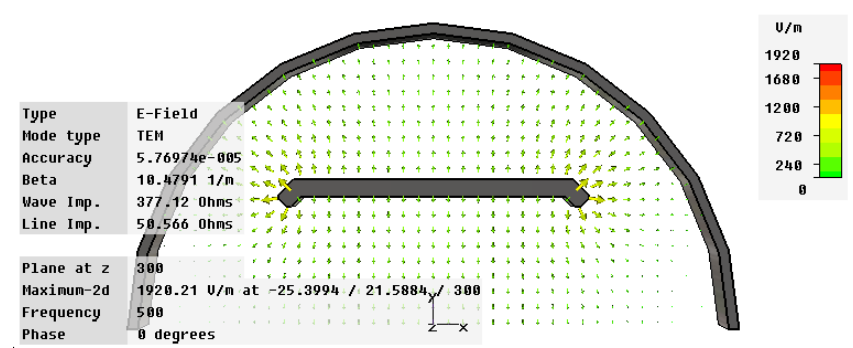

Figure 4.5: Odd TEM mode along stripline. The characteristic impedance is $50.6 \Omega$.

We also calculate the even TEM mode, shown as in Figure 4.6. The characteristic impedance is calculated to be $68.4 \Omega$, which is different than the odd mode. The image charge induced on the kicker electrode is negligible compared with the external power fed into the kicker. So the dominant mode in the kicker is the odd TEM mode and we can neglect the impedance of the even mode. However, when the requirement of impedance matching becomes more strict, such as [22], one needs to mach both even and odd mode.

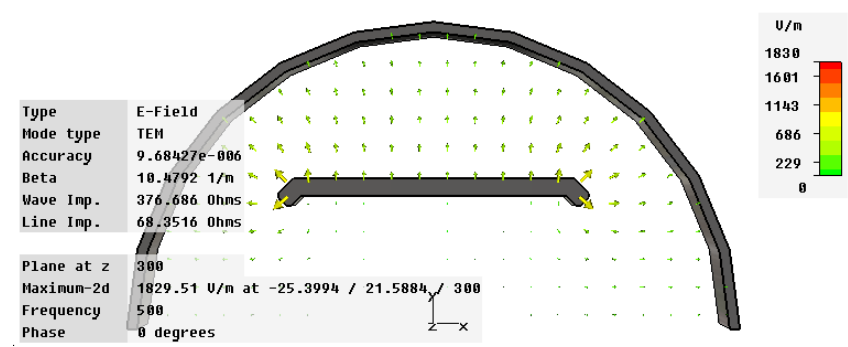

Figure 4.6: Even TEM mode along stripline. The characteristic impedance is $68.4 \Omega$. 


\section{Frequency Response}

To calculate the frequency response of the kicker, as well as the real time response of the kicking field, we build a 3D model of the kicker in Microwave Studio. Both upstream and downstream ports are approximated by coaxial metallic cylinders with $50 \Omega$ characteristic impedance. Ceramic standoffs are added to support and align the stripline electrodes. We also consider the tapering on each ends and the tube connected to a vacuum pump. A perspective view of the kicker is shown in Figure 4.7 and a side view with tapering in shown in Figure 4.8.

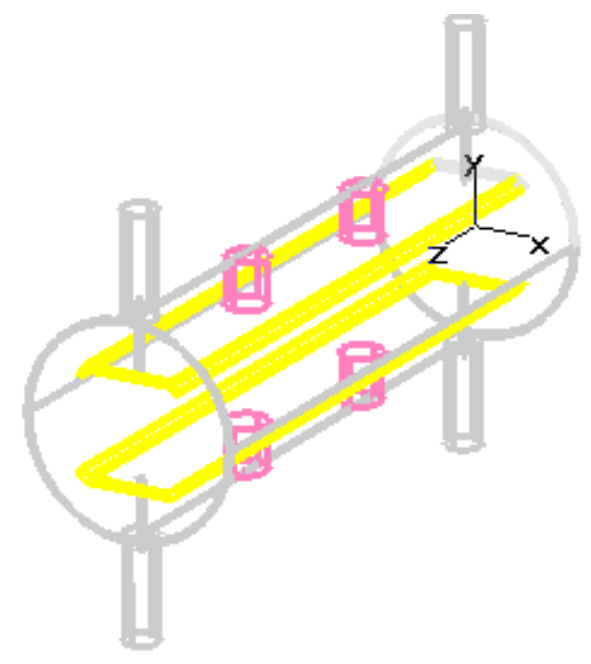

Figure 4.7: Perspective view of kicker. The yellow part is the copper electrodes and the pink part is the ceramic standoff. Here the tapering and the vacuum pump tube are not shown.

Figure 4.9 shows the input pulse from downstream port in the Microwave Studio simulation, with $10 \mathrm{~ns}$ rise time, $10 \mathrm{~ns}$ fall time and $50 \mathrm{~ns}$ flat top. It is an approximation of the real input pulse. Figure 4.10 shows its corresponding discrete fourier transform (DFT) frequency spectrum. We can see its frequency range is mainly in the 


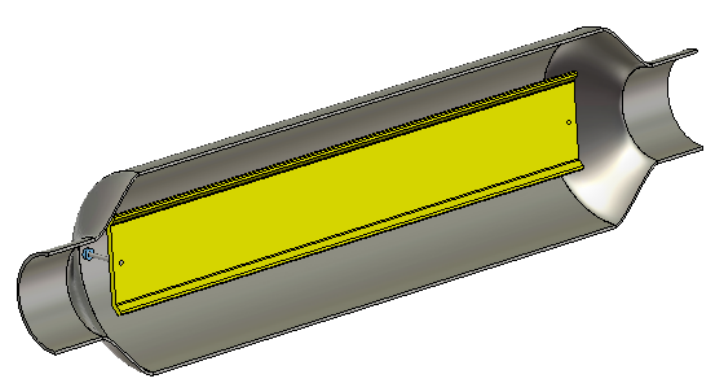

Figure 4.8: Side view of kicker with tapering. The tapering reduces the discontinuity between kicker vacuum chamber and beam pipe.

region no higher than $200 \mathrm{MHz}$, thus we want the kicker to have a good transmission efficiency up to $200 \mathrm{MHz}$.

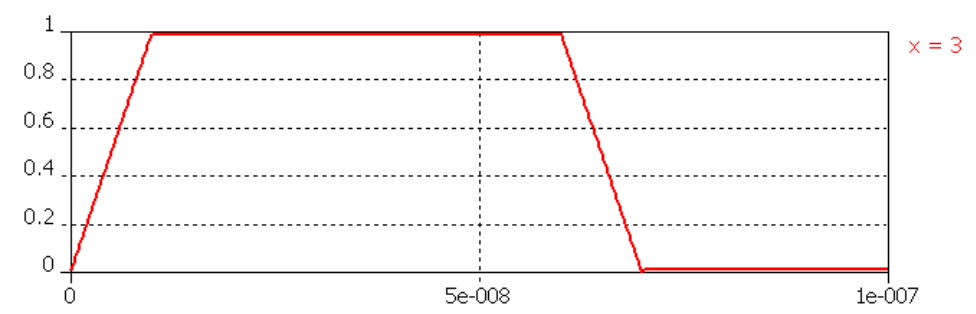

Figure 4.9: Input pulse signal.

The frequency response of the kicker is characterized by the scattering parameter (S-parameter). The reflection coefficient $\mathrm{S}_{11}$ indicates how much power is reflected back to the input port. The transmission coefficient $\mathrm{S}_{21}$ shows how much power is transmitted to the port of dummy load. The S-parameters calculation result from Microwave Studio is shown in Figure 4.11. We can see under $200 \mathrm{MHz}, \mathrm{S}_{11}$ is all below $-20 \mathrm{~dB}$, which means little power reflection in this design. 


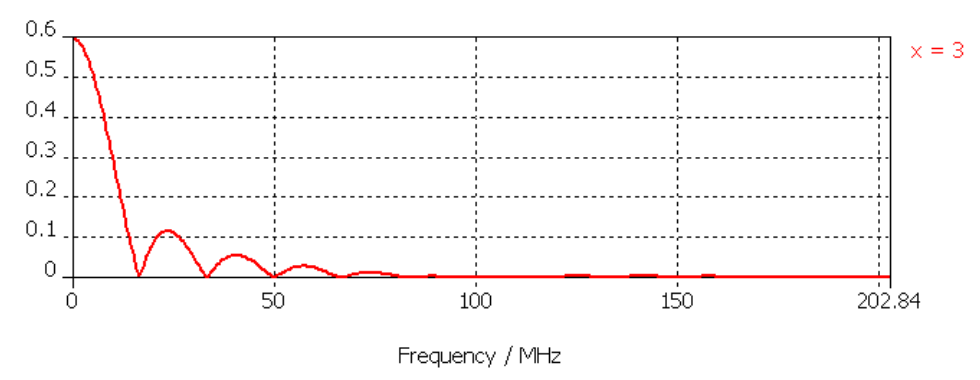

Figure 4.10: DFT of the input signal.

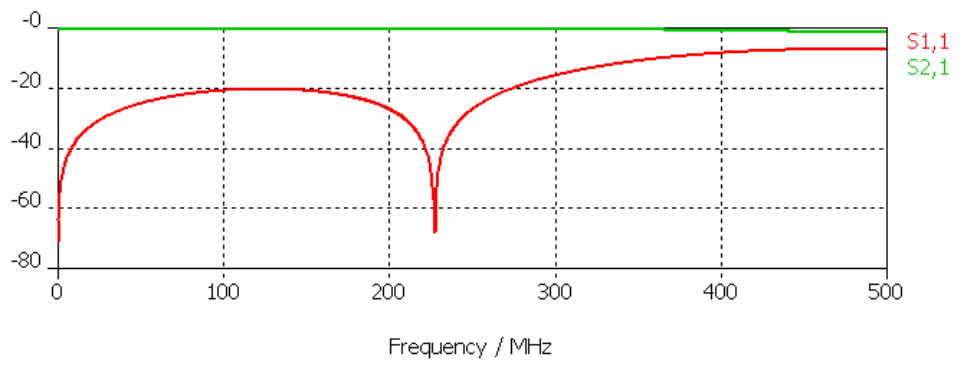

Figure 4.11: The scattering parameters between pulse port and dummy load port.

\section{Kicking Field}

One important requirement of the kicker is the uniformity of the kicking filed. In the previous 2D Poisson calculation, we have already checked the kicking field along the transverse center for the initial design. From Microwave Studio, we can calculate the kicking field along both transverse and longitudinal axes of the kicker. The kicking field of the updated design are shown in Figure 4.12 and 4.13. They are both within the design requirement and give an overall good uniformity. 


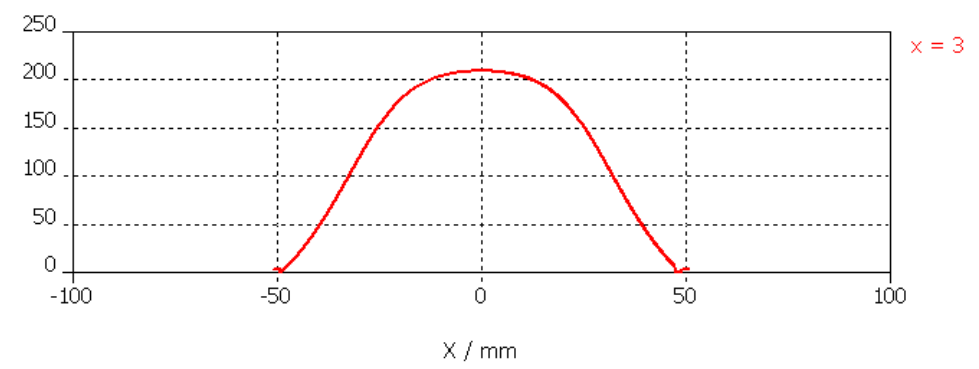

Figure 4.12: Electric field along the transverse center of kicker.

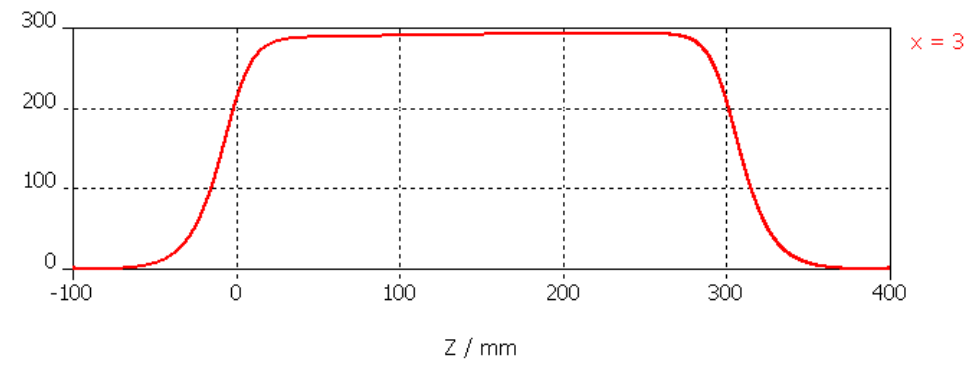

Figure 4.13: Electric field along the beam line center of kicker.

\section{Real Time Response}

With the transient mode of Microwave Studio, we can calculate the output signal and the reflected signal in the time domain. This calculation is more straightforward to check whether the kicker operates fast enough to establish a stable kicking field within 10 ns. The result is shown in Figure 4.14. We can see that:

1. There is a $2 \mathrm{~ns}$ delay between the input pulse and output pulse. This is due to the finite length of the kicker. Considering this delay, the rise time of the power supply should be within 8 ns to ensure a 10 ns rise time of the whole kicker.

2. The reflected signal is very small, verifying the good transmission property of the kicker design. 


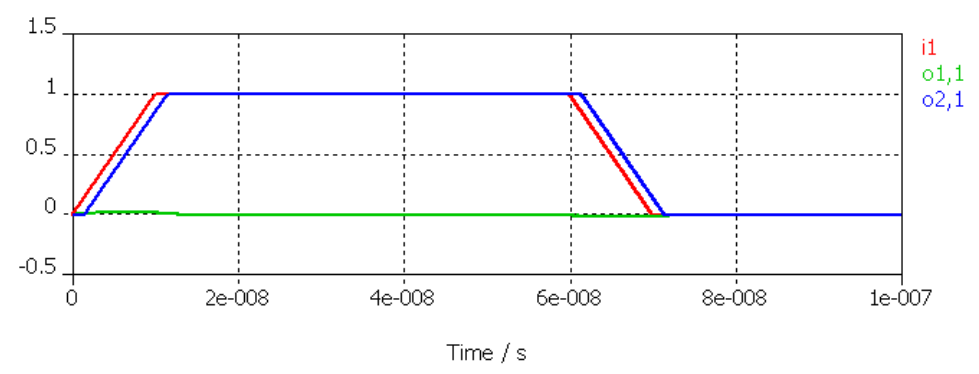

Figure 4.14: Input signal (red) at pulse port, output signal (blue) at dummy load port and reflected signal (green) at pulse port.

\subsection{Requirement of Power Supply}

After determining the kicker strength requirement and the electrode design of the kicker, we can calculate the parameters of the kicker power supply.

The kicker voltage $V_{\perp}$ can be expressed as [22]:

$$
V_{\perp}=\frac{h \cdot E_{b}(\mathrm{eV})}{2 g l} \times \theta
$$

where $h$ is the kicker gap, $E_{b}$ the beam energy, $g$ the coverage factor, $l$ the kicker length and $\theta$ the kicking angle. For our current design, $l=30 \mathrm{~cm}, h=4.76 \mathrm{~cm}$. With the width of the stripline $W=5.08 \mathrm{~cm}$, the coverage factor $g$ is:

$$
g=\tanh \left(\frac{\pi \times W}{2 \times h}\right)=0.93
$$

For a $E_{b}=50 \mathrm{MeV}$ beam, the minimum kick angle $\theta_{\min }$ of $10 \mathrm{mrad}$ requires voltage:

$$
V_{\perp}=\frac{4.74 \times 50 \times 10^{6}}{2 \times 0.93 \times 30} \times 10 \times 10^{-3}=42.5 \mathrm{kV}
$$

In the same way, we can also calculate the voltage requirement of the maximum kick angle $\theta_{\max }$ of $23 \mathrm{mrad}$, which is $97.7 \mathrm{kV}$.

The voltage on each electrode is half of the kicking voltage but with opposite polarity, with the magnitude in the range of $22 \mathrm{kV}$ to $49 \mathrm{kV}$. Such a high voltage 
requirement imposes a challenge on the vacuum feedthrough. The feedthrough has a $50 \Omega$ impedance and can withstand high voltage up to $25 \mathrm{kV}$ and ultra high vacuum sealing. At the time of this thesis, such a feedthrough is not commercially available, but has been successfully developed by [23] in accelerator community.

The shunt impedance of the kicker can be calculated as:

$$
R_{s}(\omega)=2 Z_{c}\left(\frac{2 c g}{\omega h}\right)^{2} \sin ^{2}\left(\frac{\omega l}{c}\right)
$$

where the $Z_{c}$ is the characteristic impedance of the stripline structure, which is $50 \Omega$ for our kicker, $\omega$ the frequency and $c$ the speed of light. This is the general expression for the stripline structure, no matter if it is a kicker or a BPM. For our pulse power, the wavelength of its frequency spectrum is much smaller compared to the kicker length, so $\sin \left(\frac{\omega l}{c}\right) \approx \frac{\omega l}{c}$. Thus

$$
R_{s}(\omega) \approx 2 Z_{c}\left(\frac{2 g l}{h}\right)^{2}=1.37 \times 10^{4} \Omega
$$

With the shunt impedance $R_{s}$, we can calculate the peak power on each electrode $P_{k}=V_{p}^{2} / R_{s}$, where $V_{p}=V_{\perp} / 2$, for different kicking voltage. After determining the repetition rate of the kicker, we can also determine the average power. These figures are important to estimate the heat dissipation of the dummy load. 


\section{Chapter 4}

\section{Longitudinal Beam Characterization}

In ALPHA, we use a wall gap monitor (WGM) from CIS to measure the beam current in both the injection and extraction beam line. With this wide band WGM, we have carried out several measurements in ALPHA:

1. In the injection beam line, we have measured the beam current and beam charge. From the longitudinal beam profile, we have detected the $2.856 \mathrm{GHz}$ s-band LINAC micro pulse structure due to the klystron modulation.

2. By scanning the dipole in the injection line, we have measured the longitudinal phase space of the LINAC electron beam.

3. In the extraction beam line, we have measured the longitudinal beam profile and detected the suppression of the $2.856 \mathrm{GHz}$ micro pulse structure, thus verified the debunching performance of ALPHA storage ring 


\subsection{The wide band Wall Gap Monitor}

The WGM used in CIS is designed and built by Fermilab [24]. Its sketch and the equivalent circuit are shown in Figure 4.1.
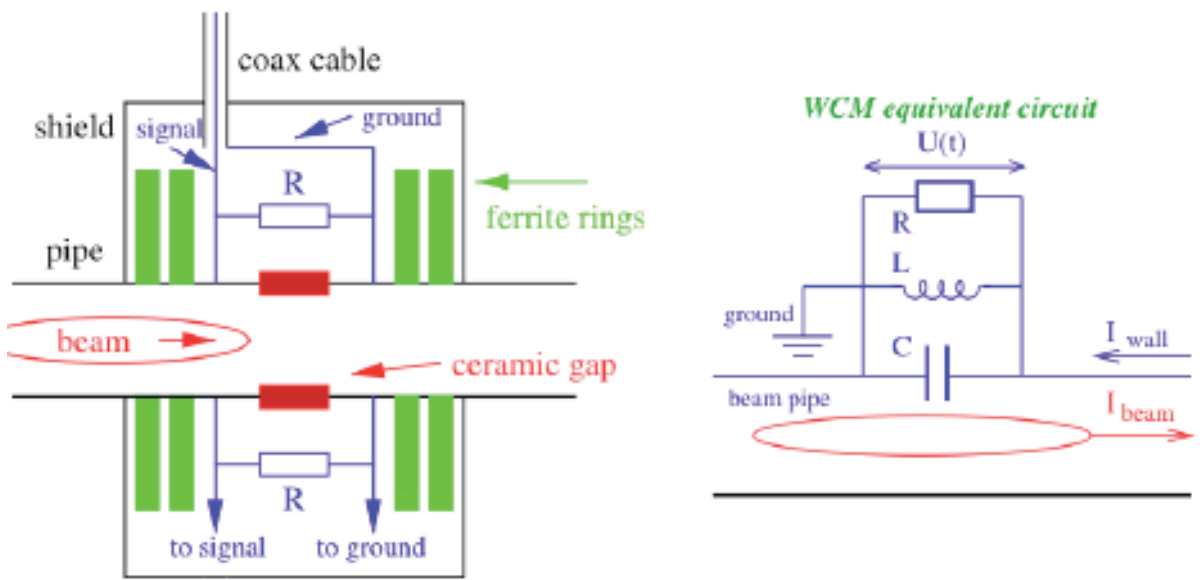

Figure 4.1: The sketch structure and equivalent circuit of the WGM [25]

The WGM can be simplified as a parallel RLC circuit, whose impedance can be calculated as:

$$
\frac{1}{Z}=\frac{1}{R}+\frac{1}{i \omega L}+i \omega C
$$

Thus

$$
Z=\frac{i \omega L}{1+i \omega L / R-(\omega L / R) \cdot(\omega R C)}
$$

For the low frequency region $\omega \ll R / L$, the circuit is inductive:

$$
Z=i \omega L
$$

For the high frequency region $\omega \gg 1 / R C$, the circuit is is capacitive:

$$
Z=1 / i \omega C
$$


For the median frequency region $R / L \ll \omega \ll 1 / R C$, the circuit is resistive:

$$
Z \approx R
$$

The proper working range for the WGM is in the median region $R / L \ll \omega \ll$ $1 / R C$, where the circuit is almost purely resistive and the beam current is linearly proportional to the voltage across the resistor.

The bandpass effect of the WGM will distort the shape of the signal when its frequency is out of or at the margin of the proper working range. The effect is shown in Figure 4.2:

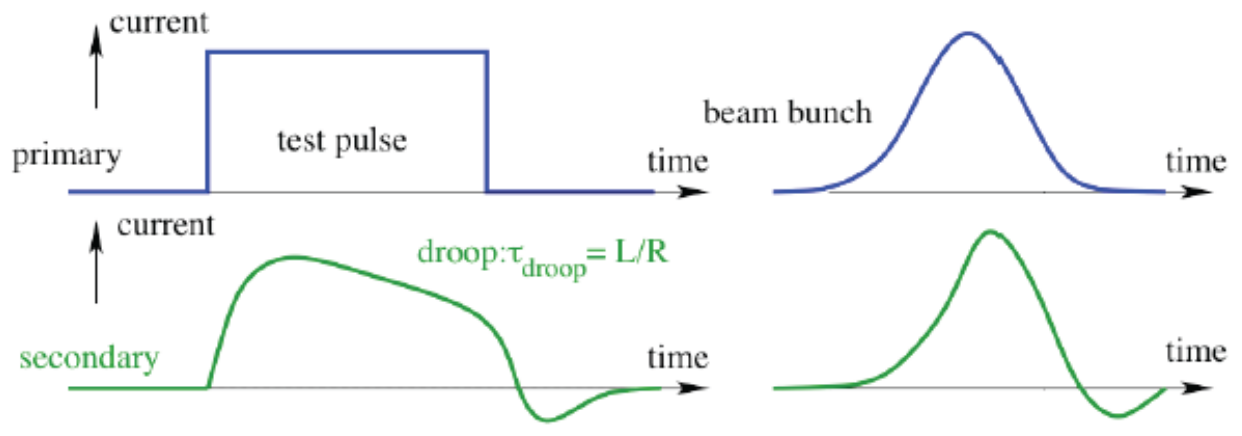

Figure 4.2: The comparison of the beam shape and the WGM signal shape due to the bandpass effect. The left is a square pulse. The right is a more realistic gaussian shape.

In the frequency domain, the bandpass region is described by the low frequency limit $\omega_{\text {low }}=R / L$ and the high frequency limit $\omega_{\text {high }}=1 / R C$. Correspondingly, it can be characterized by rise time $t_{\text {rise }}$ and the droop time $t_{\text {droop }}$ in the time domain:

$$
t_{\text {rise }}=\frac{\ln 0.9-\ln 0.1}{\omega_{\text {high }}} \approx \frac{1}{3 f_{\text {high }}}
$$




$$
t_{\text {droop }}=\frac{\ln 0.9-\ln 0.1}{\omega_{\text {low }}} \approx \frac{1}{3 f_{\text {low }}}
$$

Any pulse variation faster than $t_{\text {rise }}$ or slower than $t_{\text {droop }}$ will be hard for the WGM to recover.

To achieve a low cut off frequency, several ferrite toruses are put near the ceramic gap to increase the inductance $L$, as shown in Figure 4.3. There are also sponges to absorb the RF field in the copper chamber, which is induced by the image current.

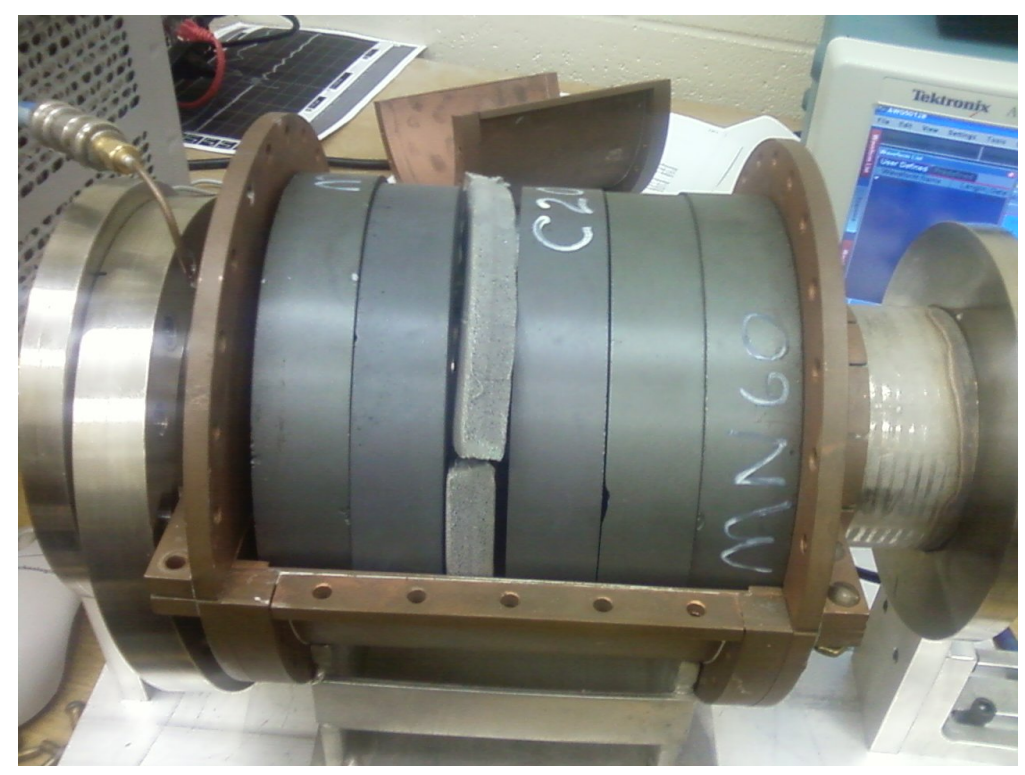

Figure 4.3: The picture of the WGM, showing the ferrite torus and the RF absorber.

There are $80120 \Omega$ resistors soldered around the ceramic gap in parallel with equal space, as shown in Figure 4.4. Thus the total image current doesn't depend on the position or the transverse profile of the beam. It is determined exclusively by the total beam current. 


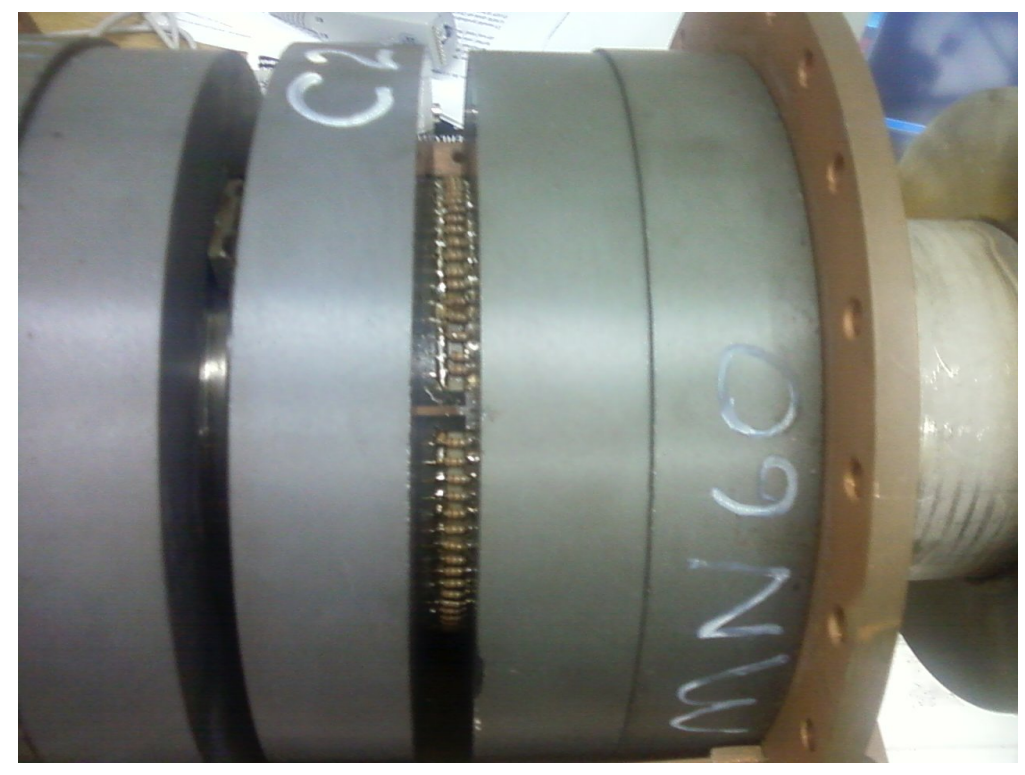

Figure 4.4: The picture of the WGM, showing the resistors around the ceramic gap.

\subsection{Bench test of WGM}

Before putting the WGM into the accelerator, we did the bench test to:

1. Calibrate the effective impedance $R$ of the WGM.

2. Measure the bandwidth of the WGM.

The bench test setup is shown in Figure 4.5. A thin metallic wire is stretched through the WGM, with one end connected to the function generator and the other end to the $50 \Omega$ terminator. The function generator sends the pulse signal across the wire to simulate the beam current. The output signal is measured by the oscilloscope. 


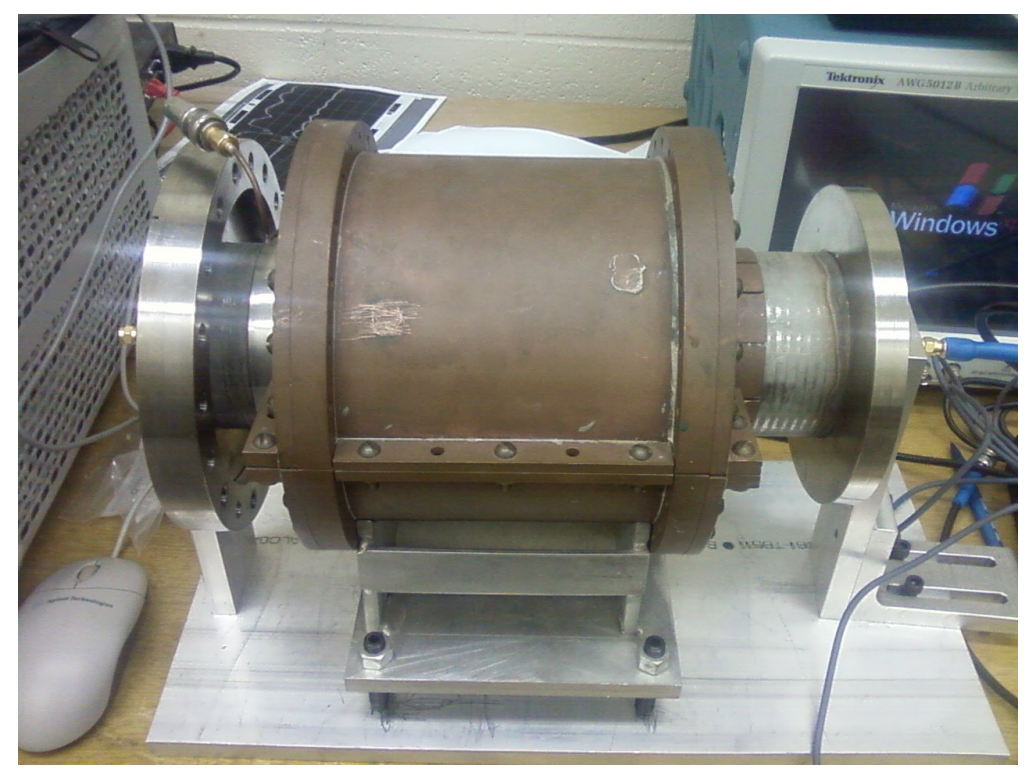

Figure 4.5: The picture of the WGM bench test setup.

\subsubsection{Effective impedance $R$}

In the proper working range, the WGM is almost purely resistive. The voltage measured across the ceramic gap is proportional to the beam current:

$$
\text { Beam current } I=\frac{\text { Voltage across the ceramic gap } V}{\text { Effective impedance } R}
$$

The effective impedance $R$ can be calibrated as:

Effective impedance $R=\frac{\text { Integration of the function generator signal }}{\text { Integration of the measured signal }} \cdot 50 \Omega$

It should be a constant in the proper working frequency range. In the calibration, we change the input pulse shape, length and magnitude, measure the output signal and derive the $R$ by linear fitting. One function generator input signal and its corresponding WGM output signal are shown in Figure 4.6.

To test the sensitivity of the signal to the beam position in the beam pipe, we moved the wire position around the beam pipe center and tilt the wire in different 


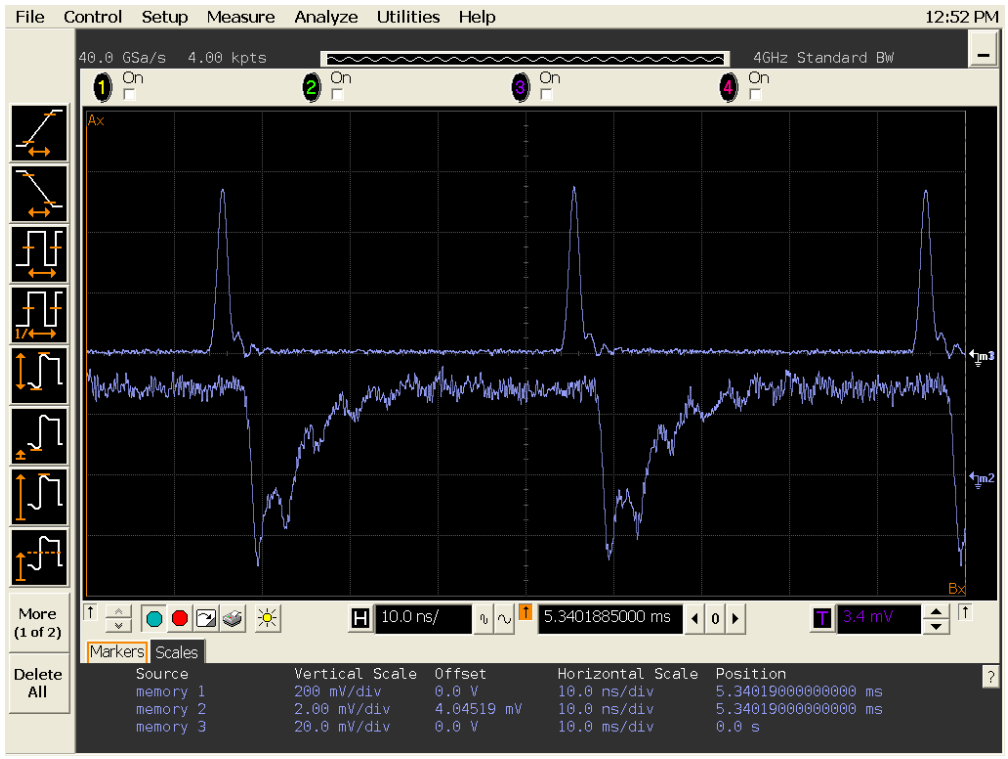

Figure 4.6: One of the bench test signals. The upper is the input signal from the Function Generator, the lower is the output signal from the detector port. The polarity of the output signal is determined by the direction of the signal on the wire. 
direction, as shown in Figure 4.7. The WGM signal shows undetectable difference for all these different situations.

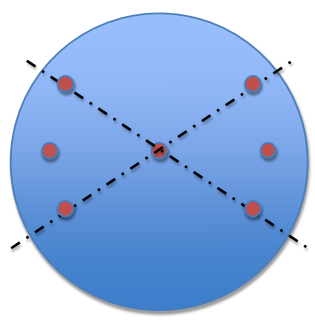

(a)

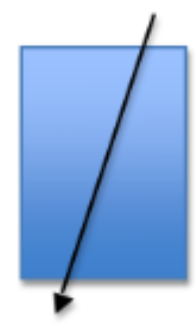

(b)

Figure 4.7: WGM signal sensitivity to the beam position and orientation

An example of the linear fitting is shown in Figure 4.8. Combining all the tests with different input signal patterns with different magnitude, the WGM effective impedance is calibrated to be $2.1 \Omega$.

\subsubsection{Bandwidth}

The high frequency limit has been measured in [26]. The result is $3 \mathrm{GHz}$. It is higher than the $2.856 \mathrm{GHz}$ micro pulse frequency, thus the WGM can detect the micro pulse signal. However, it is not high enough to recover the complete shape of the micro pulse, which requires up to tens of $\mathrm{GHz}$ working frequency range. Due to the limit of Network Analyzer performance and mismatching of the test bench, we can't verify this high frequency limit ourselves but only trust this measurement.

To determine the low frequency limit, we measure the time response of a square wave, as shown in Figure 4.9.

The measured coordinates of point A and B are: 


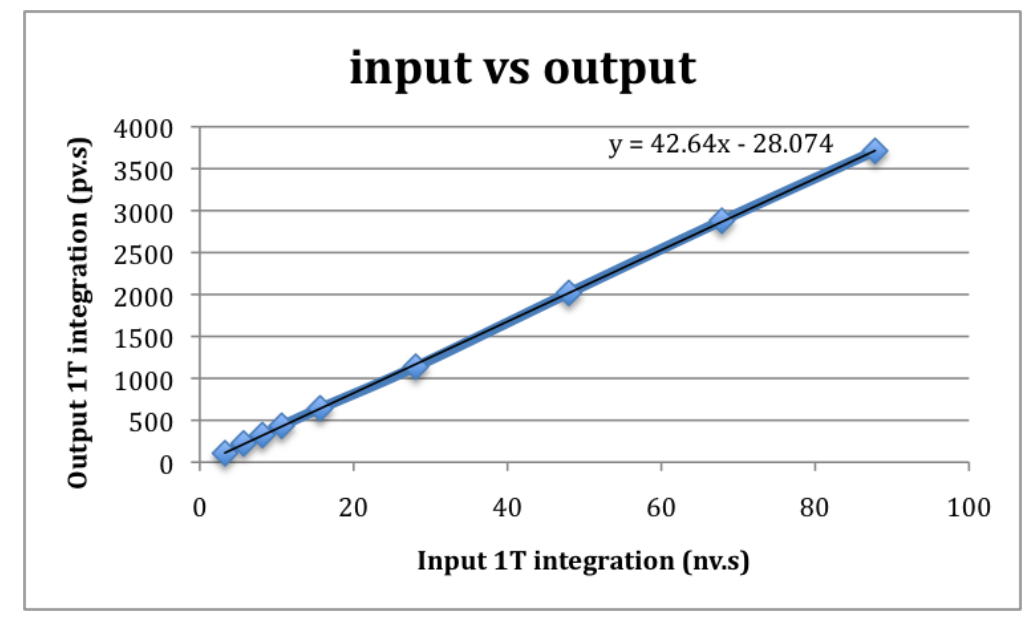

Figure 4.8: One example of the linear fitting of the input signal and output signal of the bench test. The slope is used to derive the effective impedance of the WGM. We have tested different input signal patterns with different strengths.

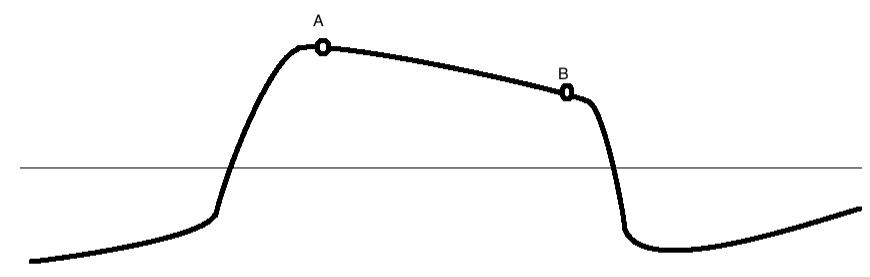

Figure 4.9: The WGM droop time measurement. 


$$
\begin{array}{ll}
t_{A}=-1.443 \mu \mathrm{s} & V_{A}=41.16 \mathrm{mV} \\
t_{B}=6.624 \mu \mathrm{s} & V_{B}=33.28 \mathrm{mV}
\end{array}
$$

From the definition of droop equation of a constant signal:

$$
V_{B}=V_{A} \times e^{-\left(t_{B}-t_{A}\right) / \tau_{d r o o p}}
$$

we can calculate the droop time:

$$
\tau_{\text {droop }}=\left[\frac{\ln \left(V_{A} / V_{B}\right)}{t_{B}-t_{A}}\right]^{-1}=41.06 \mu \mathrm{s}
$$

and the low frequency limit $f_{\text {low }}$ :

$$
f_{\text {low }}=\frac{1}{2 \pi \tau_{\text {droop }}} \approx 3.88 \mathrm{kHz}
$$

\subsection{Measurement of the injected beam}

Part of ALPHA injection beam line layout is shown in Figure 4.10. After coming out from a S-band LINAC, the beam is bent by two 45-degree dipole, with a slit in between to reduce the momentum spread. The WGM is placed right after the second dipole. With the WGM, we can measure the charge and the longitudinal profile of the injected beam.

\subsubsection{Signal shielding and amplification}

Due to the noisy environment and the small beam current passing through the slit, suppressing the noise and amplifying the signal are the keys to get good measurement. To shield the noise, especially the noise from the Klystron nearby, we use the Helix cable for all the connection between the WGM and the scope, as well as the amplifier and the power source. 


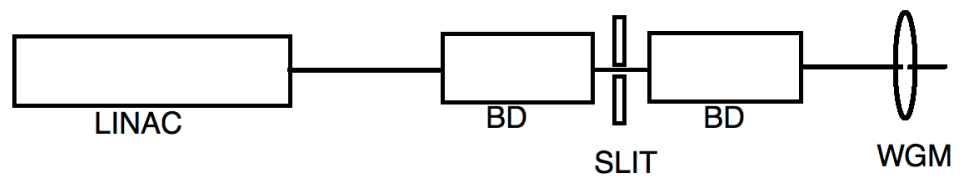

Figure 4.10: The ALPHA injection beamline layout from LINAC to WGM.

After the shielding, we can detect the injected beam current signal, as shown in Figure 4.11. The $2.856 \mathrm{GHz}$ peak is also obvious on the FFT (Fast Fourier Transform). However, the signal is weak. And if we zoom into the micropulse time scale, we can't tell the $2.856 \mathrm{GHz}$ micro structure.

To improve the signal, we use the RF amplifier to magnify the signal. As shown in Figure 4.12, both the macro and micro pulse become clearer. The micro pulse signal verifies our previous statement that due to the high frequency cut off, we can not measure the micro pulse shape but only $2.856 \mathrm{GHz}$ oscillating structure. Due to the low frequency cut off from RF amplifier, the marco pulse shape is distorted and we need to be careful about this when we process the signal.

\subsubsection{Signal processing with modulation-demodulation scheme}

From the WGM signal, we want to derive the charge and the longitudinal profile of the injected beam. The raw data is very noisy even after the amplification, thus we need to do more signal processing to get the useful information.

The injected beam is a long series of very short pulses of a few pico seconds. The FFT of such a signal is composed of the DC and several higher order harmonics peaks. 


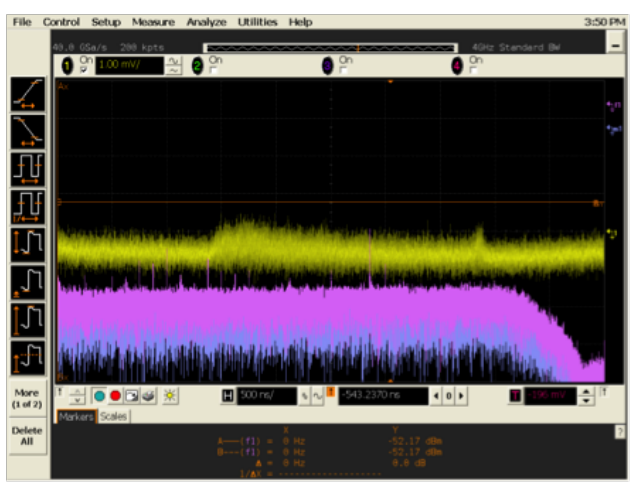

(a) Macropulse

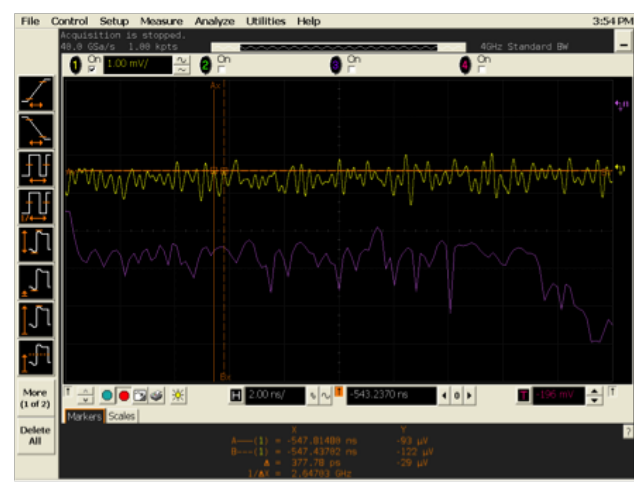

(b) Micropulse

Figure 4.11: WGM signal of injected beam without amplifier. The yellow trace is the beam current measurement, the red trace is the FFT of the yellow trace.

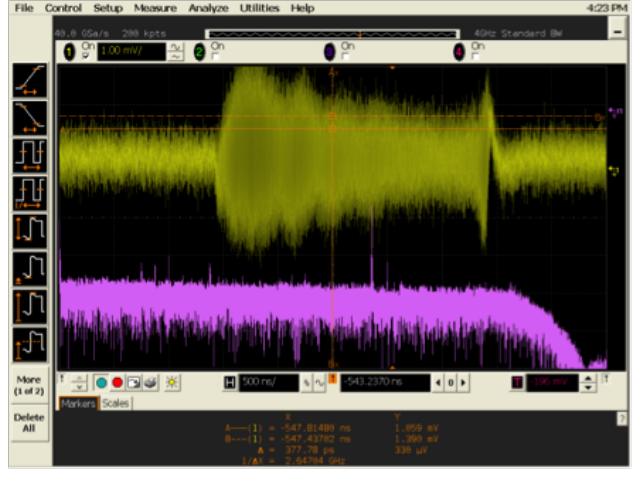

(a) Macropulse

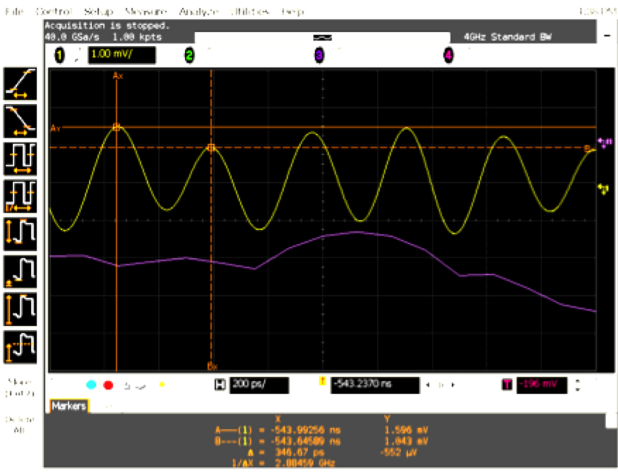

(b) Micropulse

Figure 4.12: WGM signal of injected beam with amplifier. 
The width of the peak is narrow and the magnitude drops slowly from the DC peak to higher order peaks. Utilizing this character of the injected beam, we develop a modulation-demodulation scheme for the signal processing:

1. Do the FFT on the amplified WGM signal and identify the DC and $2.856 \mathrm{GHz}$ peak on the frequency spectrum. Notice that the DC peak is not the real DC part of the original beam signal. Due to the band limit of amplifier, the DC strength has been reduced. Lots of noise and signal level shift are also included in this DC peak. The $2.856 \mathrm{GHz}$ peak is more accurate since there is almost no noise at such a high frequency.

2. Apply a bandpass filter from $2.7 \mathrm{GHz}$ to $3.0 \mathrm{GHz}$ to eliminate the DC peak and keep the $2.856 \mathrm{GHz}$ peak. This is equivalent to keep the modulation the beam signal of $2.856 \mathrm{GHz}$. The information of the total charge is also contained inside the $2.856 \mathrm{GHz}$ spectrum.

3. On the frequency spectrum, shift the $2.856 \mathrm{GHz}$ peak to DC. The signal is demodulated and the DC strength is recovered.

4. Do the Inverse FFT on the frequency spectrum to get the signal in time domain. The longitudinal beam shape is recovered. From the time integration of the voltage signal, with the WGM effective impedance we have calibrated, we can calculate the total charge of the macro pulse.

Before applying this modulation-demodulation scheme to the real experimental data, we first carry out a test in Matlab with a pseudo signal, as shown in Figure 4.13. The Matlab calculation of the macro pulse time integration is $4.678 \times 10^{-9}$ Vs.

Due to the bandwidth limit of WGM, we apply a low pass filter with the frequency cutoff at $3.5 \mathrm{GHz}$ on the pseudo signal. This is the raw signal we expect to measure 


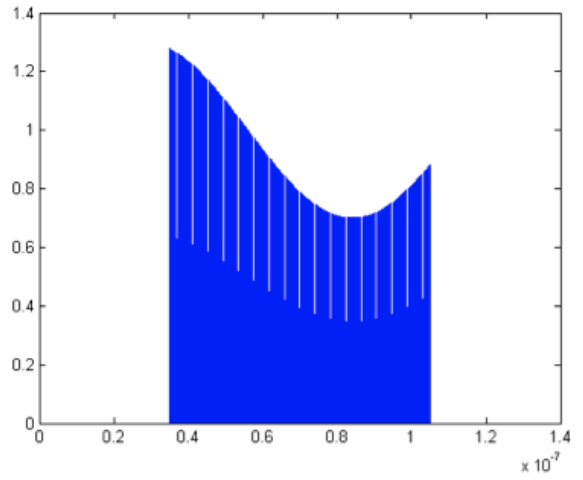

(a) Macropulse

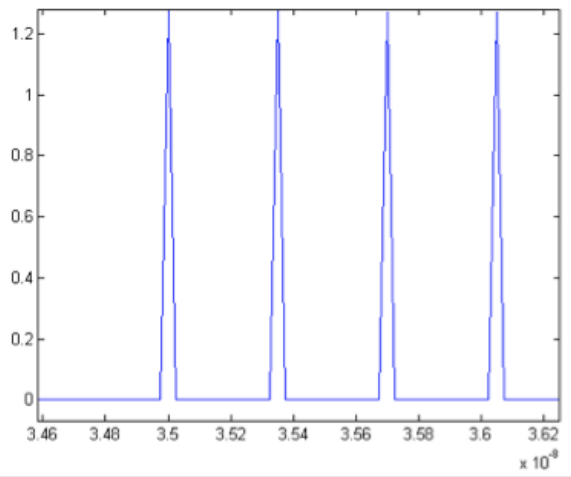

(b) Micropulse

Figure 4.13: The pseudo signal constructed in Matlab to simulate the LINAC beam pulse. The micro pulse period is 350 ps. The sample rate is $40 \mathrm{GS} / \mathrm{s}$, in accordance with the oscilloscope we use in the measurement. 
from the WGM, as shown in Figure 4.14. We can see that due to the cutoff, we can not tell the detailed micro pulse length of shape, but only a sinusoidal oscillation with a 350 ps period. This is consistent with the measurement result in Figure 4.12. We also see the low frequency cutoff in the experiment data and this is due to the amplifier. The Matlab calculation of the macro pulse time integration is $4.967 \times 10^{-9}$ Vs.

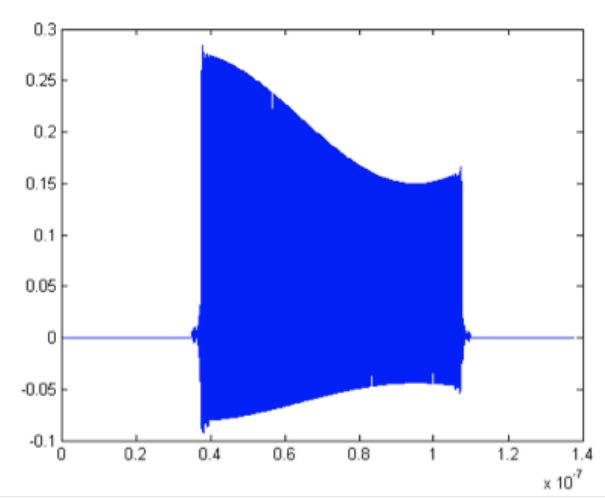

(a) Macro pulse

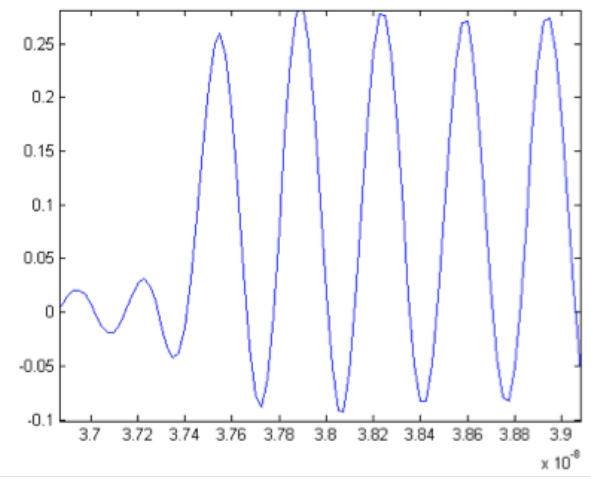

(b) Micro pulse

Figure 4.14: The pseudo signal after the WGM bandwidth limit cutoff.

Following the above procedure, we carry out the modulation-demodulation on the pseudo signal. The results are shown in Figure 4.15. The Matlab calculation of the time integration of the demodulated signal is $4.868 \times 10^{-9} \mathrm{Vs}$, which agrees with the original signal within $5 \%$. Thus we have proved the validity of this data processing scheme for calculating the beam charge. The macropulse shape is also preserved in the demodulated signal.

We also test several other macro pulses with different shapes and their corresponding modulation-demodulation processing results, as shown in Figure 4.16, Figure 4.17 


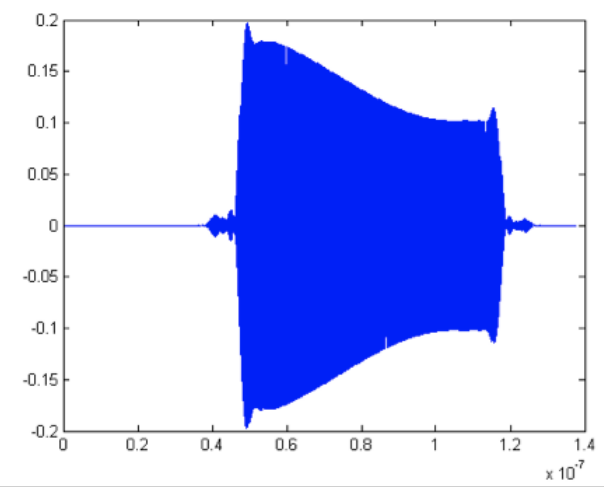

(a) Modulated signal

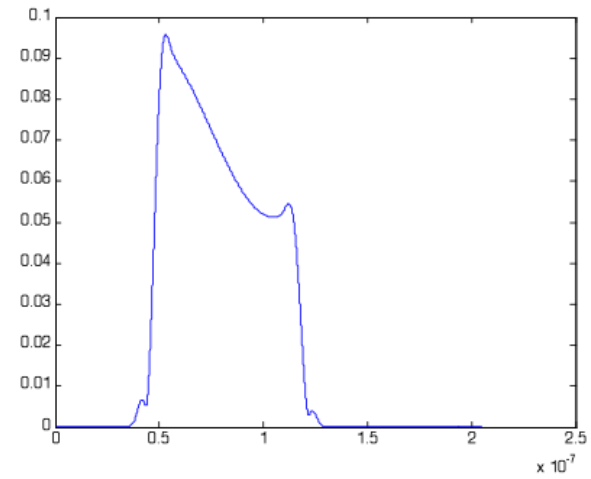

(b) Demodulated signal

Figure 4.15: The pseudo signal after the modulation and demodulation

and Figure 4.18. The time intervals of original signal and processed signal agree within $5 \%$, and the shapes of the pulse are preserved quite well.

Notice that the bandwidth of the band pass filter is $300 \mathrm{MHz}$ around the 2.856 GHz peak. This is sufficient to catch any signal variation slower than $1 / 300 \mathrm{MHz}=3.3$ ns. If the bandwidth is too narrow, we will loss some fast changing characters of the original signal.

Mathematically, we can use a delta train to illustrate this processing scheme. Assuming the beam current is:

$$
I(t)=Q \cdot f(t) \cdot g(t)
$$

where $f(t)=\sum_{m=-\infty}^{+\infty} \delta\left(t-m T_{0}\right), T_{0}=350$ ps and $g(t)$ is a nonzero slow-varying function in a $2.5 \mu$ s time window and zero everywhere outside this window. The frequency spectrum of $I(t)$ :

$$
\tilde{I}(\omega)=Q \cdot \tilde{f}(\omega) * \tilde{g}(\omega)
$$




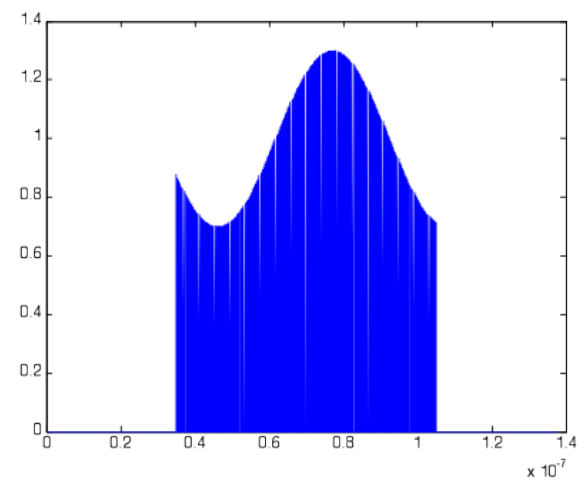

(a) Modulated signal

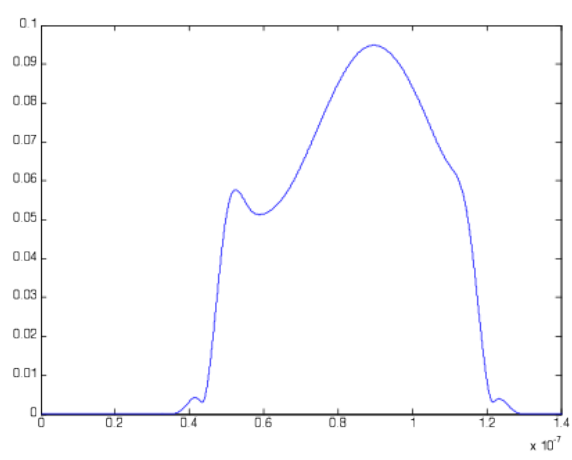

(b) Demodulated signal

Figure 4.16: Example 1: the pseudo signal and the processed signal.

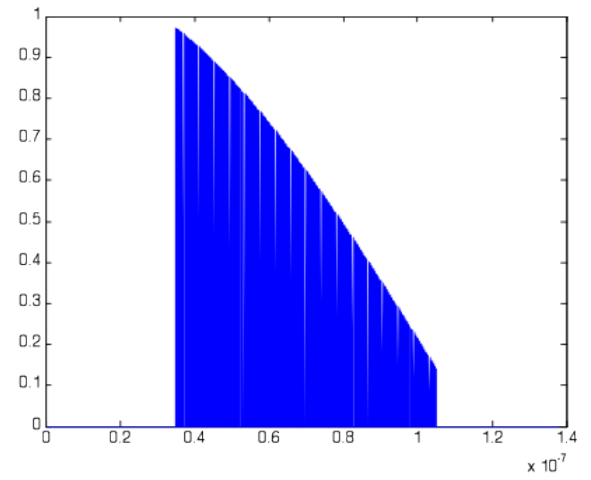

(a) Modulated signal

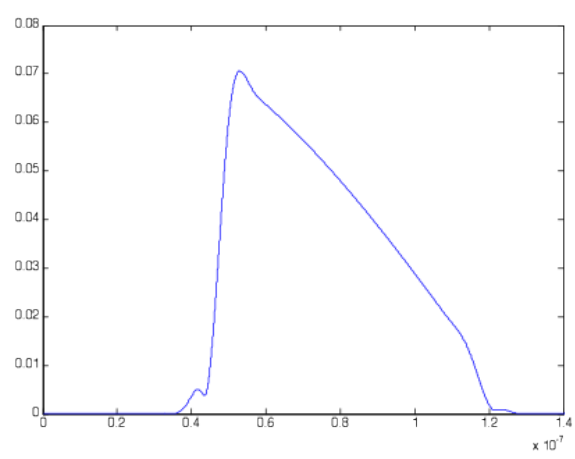

(b) Demodulated signal

Figure 4.17: Example 2: the pseudo signal and the processed signal. 


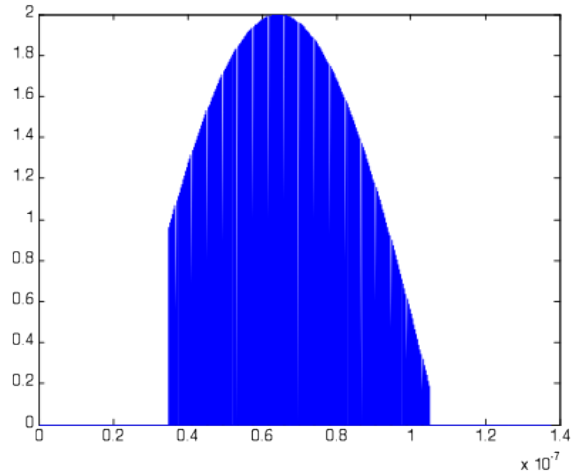

(a) Modulated signal

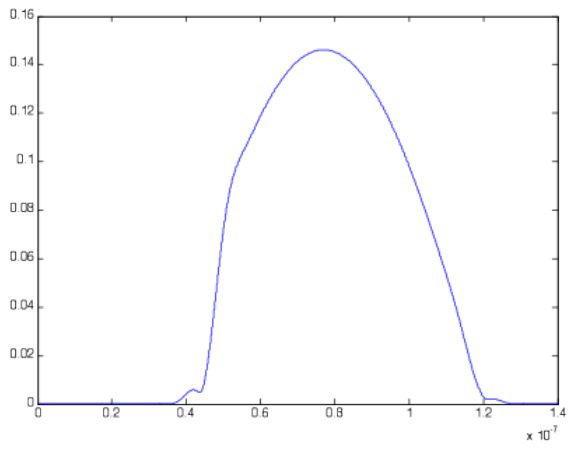

(b) Demodulated signal

Figure 4.18: Example 3: the pseudo signal and the processed signal.

where "*" stands for convolution of the spectrum function. From the property of Fourier Transform, we know that

$$
\tilde{f}(\omega)=\sum_{n=-\infty}^{+\infty} \delta\left(\omega-n \omega_{0}\right)
$$

with $\omega_{0} / 2 \pi=2.856 \mathrm{GHz}$.

The specific form of $\tilde{g}(\omega)$ depends on the shape of macro pulse, but we can predict that the range of the frequency spectrum should be at the order of $1 / 2.5 \mu s=400$ $\mathrm{kHz}$, which is much smaller than $\omega_{0}$. Thus

$$
\tilde{I}(0)=\tilde{I}\left(\omega_{0}\right)
$$

and we can recover the DC current strength from the first harmonic strength.

If $f(t)$ is not a delta train but a train of gaussian pulse with finite width $\sigma$, then $\tilde{f}\left(\omega_{0}\right) \leq \tilde{f}(0)$. The relation between the ratio $r=\tilde{f}\left(\omega_{0}\right) / \tilde{f}(0)$ and the pulse width $\sigma$ is shown in Figure 4.19. For Varian medical LINAC, usually $\sigma \leq 30$ ps, thus we can still regard $\tilde{f}\left(\omega_{0}\right) \approx \tilde{f}(0)$ and $\tilde{I}(0) \approx \tilde{I}\left(\omega_{0}\right)$. 


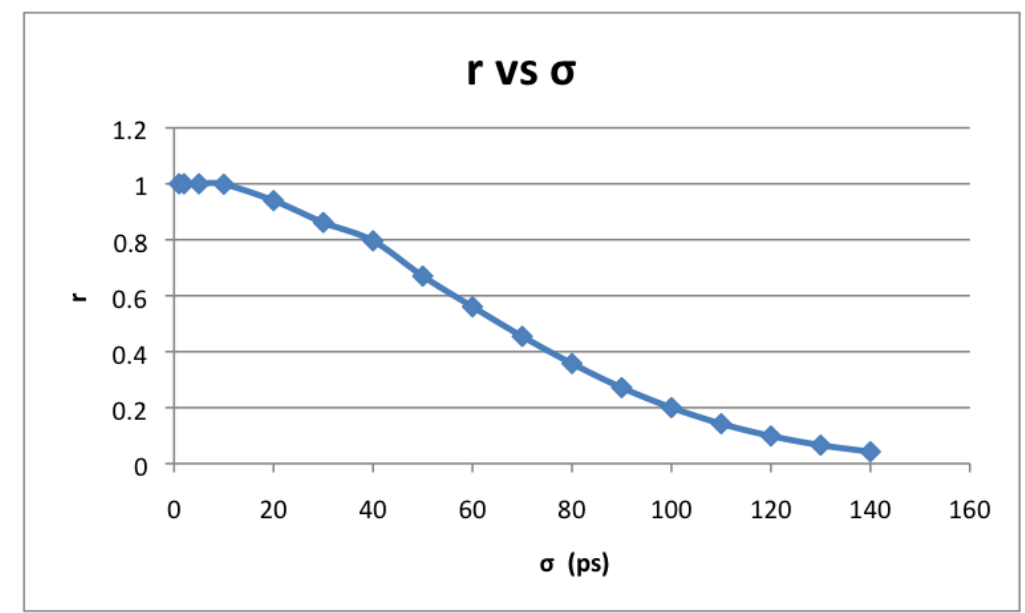

Figure 4.19: The relation between the ratio $r=\tilde{f}\left(\omega_{0}\right) / \tilde{f}(0)$ and the pulse width $\sigma$

The main advantage of this scheme is that it overcomes the DC cutoff introduced by the WGM and the amplifier, and reduces the signal noise which is mainly in the low frequency spectrum region. It is also easier to see the longitudinal beam profile from the demodulated signal.

However, this scheme assumes that the first harmonic peak strength is almost the same as the DC peak strength in the original beam signal frequency spectrum, which is true only when the length of micro pulse is less than tens of pico seconds. It is true for the beam coming out from the S-Band LINAC. But after the debunching, the micro pulse length will increase a lot with the first harmonic peak strength dropping significantly. Thus this scheme is no longer valid anymore.

\subsubsection{Charge and longitudinal profile of the injected beam}

After applying the modulation-demodulation procedure on the WGM measurement, as shown in Figure 4.12, we get the demodulated result shown as in Figure 4.20. 
With the time integration of the voltage signal and the WGM effective impedance

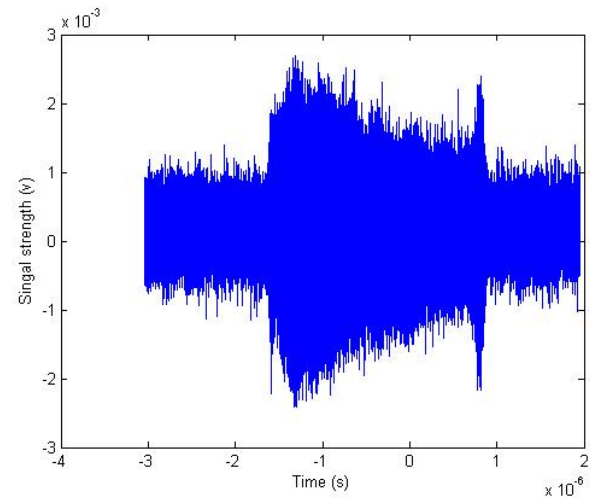

(a) Original signal imported to Matlab

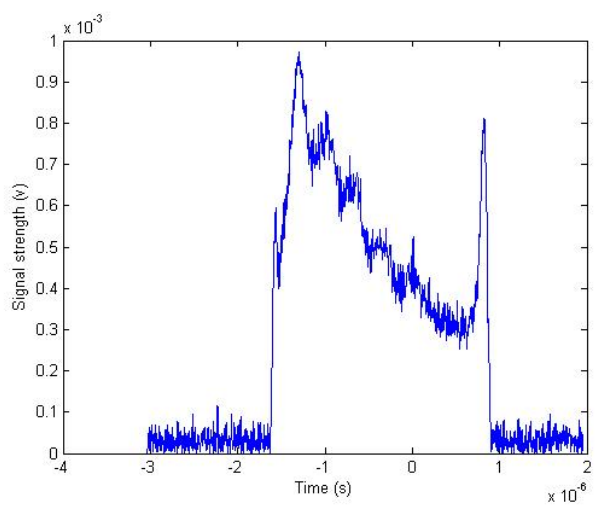

(b) Demodulated signal

Figure 4.20: The processed signal after modulation-demodulation.

calibrated before, the total charge of the injected beam is calculated to be $91 \mathrm{pC}$.

\subsection{Longitudinal tomography of the LINAC beam}

As shown in Figure 4.10, the slit selects part of the LINAC beam with the "right" energy corresponding to the bending dipole strength. By changing the dipole strength, we can select different energy parts of the LINAC beam and measure the longitudinal profile of each part. Thus we can obtain the longitudinal tomography of the LINAC beam.

\subsubsection{G4beamline simulation of the injected beam}

The bending dipole and the slit together perform the energy selection. For the beam with no transverse size or momentum and the slit with arbitrary small width, the 
calculation is easy and straight forward. However, the finite size of the beam and the transverse momentum make the case complicated. The finite size of the slit also imposes a limit on energy resolution. Thus we use the G4beamline [27] to simulate this energy selection process to see how well we can recover the beam energy distribution by this dipole scanning scheme.

G4beamline is a single-particle tracking program based on the Geant4 simulation toolkit. It is specifically designed for the simulation of beamlines. Each element in accelerator has been pre-defined and the there are several coordinate systems to place the elements along the beamline. Users don't need to dig deeply into the detailed Geant4 code and start the simulation in a straightforward way.

In G4beamline, we constructed the injection beam line from LINAC to WGM, as shown in Figure 4.21. Each magnet element is placed at the position according to the mechanical drawing of the ALPHA injection line. The quadrupole strength is set to be the same as the normal operation value.

For the beam coming out from the LINAC, the previous emittance measurement shows a transverse emittance from 5 to $10 \mu \mathrm{m}$-rad. So we assume a rms transverse size $x / z$ to be $1 \mathrm{~mm}$ and the rms transverse momentum $x^{\prime} / z^{\prime}$ to be $0.5 \%$, which makes the rms transverse emittance $\epsilon_{x} / \epsilon_{z}$ to be $5 \mu \mathrm{mrad}$. For simplicity, we assume a gaussian distribution of beam energy, with $E_{0}=22.22 \mathrm{MeV}$, which is the on-momentum energy under the normal operating condition, and $\sigma_{\mathrm{E}}=1 \mathrm{MeV}$. The energy spectrum of the injected beam we use in the simulation is shown in Figure 4.22.

First, we want to verify that during the scanning, the energy of the beam passing through the slit can be represented by the bending dipole strength. As the energy of each electron is not identical but with a distribution, we use the average value for the energy of the whole beam. The plot of nominal energy $E_{n}$ calculated by the dipole strength vs the average beam energy $E_{a v g}$ is shown in Figure 4.23. We can see with in the limit of rms spread of the beam energy, which is $1 \mathrm{MeV}$, they agree quite well. 


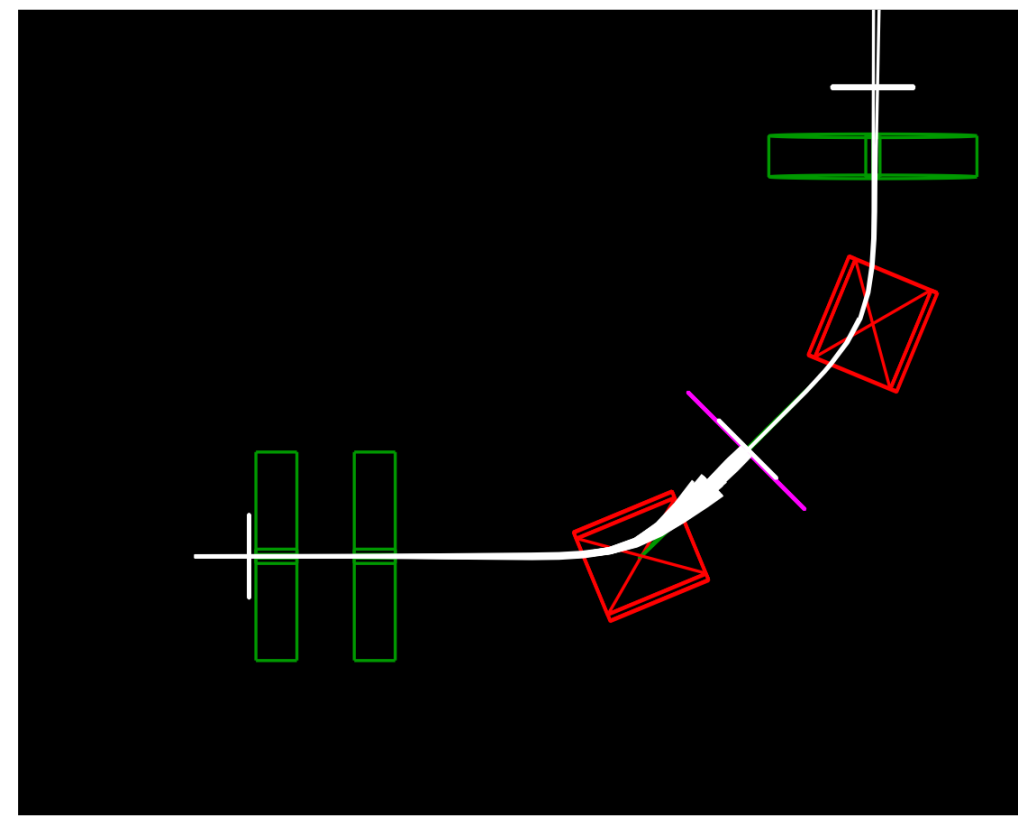

Figure 4.21: The injection beamline constructed in G4beamline.

The red parts are the bending dipoles, green parts the quadrupoles, magenta part the slit, white parts the viewers. The injected beam is also shown as while line along the beamline. The WGM is represented by a viewer to detect the beam passing through it. 


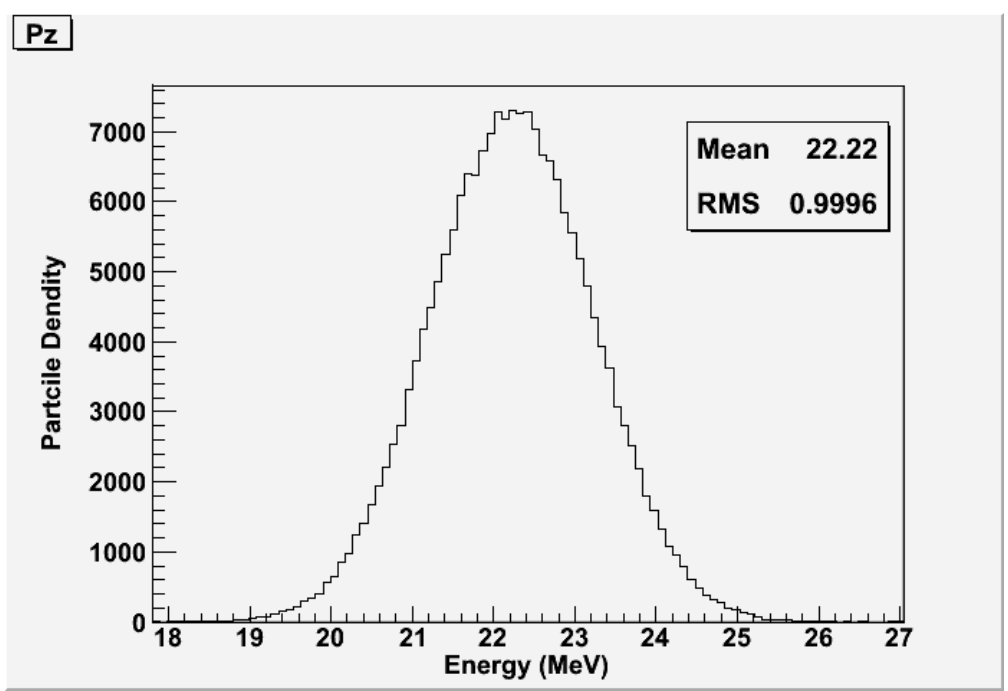

Figure 4.22: The energy spectrum of the LINAC beam. It is a gaussian distribution with an average of $22.22 \mathrm{MeV}$ and $\sigma$ of $1 \mathrm{MeV}$

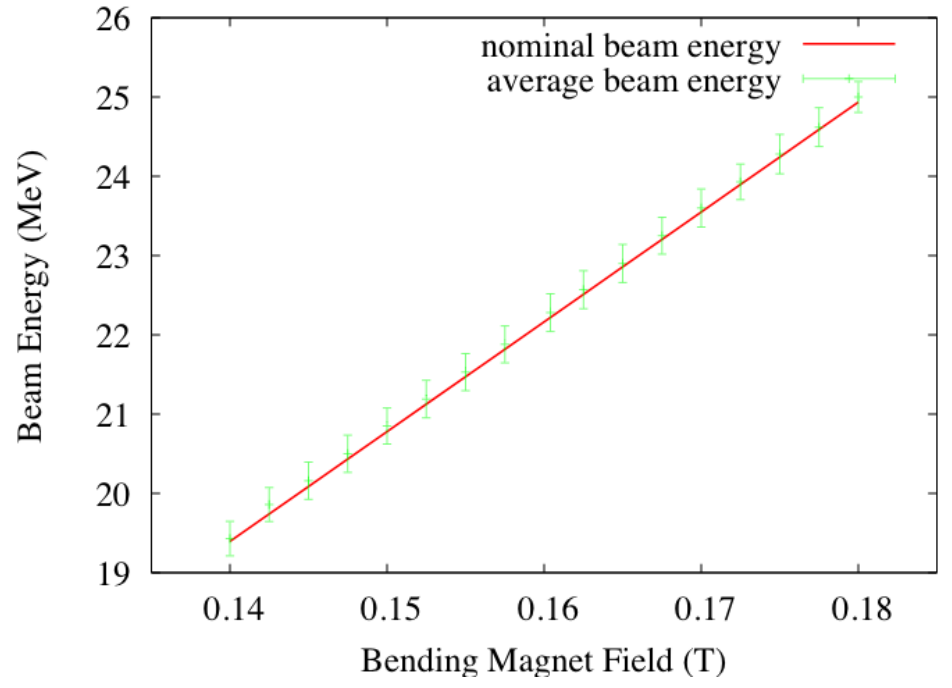

Figure 4.23: The comparison of nominal energy and the average beam energy at WGM location 
Second, we compared the energy spectrum of the LINAC injected beam and recovered result from the dipole scanning. As stated before, the LINAC beam is of a gaussian distribution centering at $22.22 \mathrm{MeV}$ with a $\sigma$ of $1 \mathrm{MeV}$. For the recovered energy spectrum, we fit it with a gaussian distribution and got a mean energy of 22.25 $\mathrm{MeV}$ and a $\sigma$ of $0.99 \mathrm{MeV}$. Thus the shape of the spectrum has been recovered quite well. However, it is difficult to recover the magnitude of the spectrum, due to the finite size of the slit and the beam loss outside the slit region.

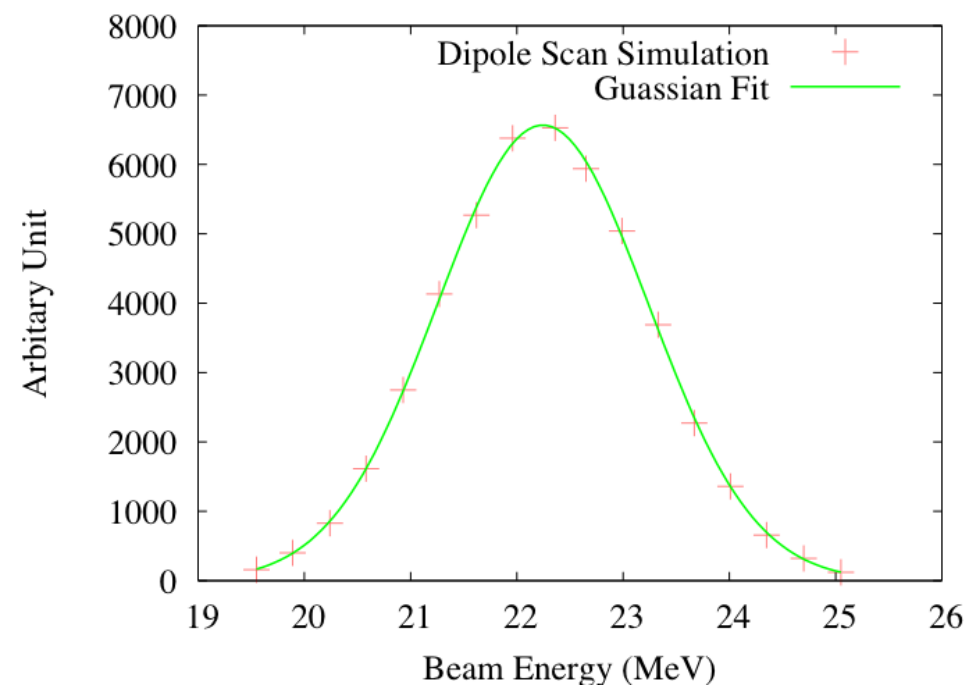

Figure 4.24: The energy spectrum recovery from dipole scanning. The data points are fitted into a gaussian distribution.

Last, the simulation also gives us a clue of the energy resolution of this scanning scheme. The $\sigma_{E}$ is about $0.13 \mathrm{MeV}$ for each scan. This limit is imposed by the finite width of the slit. 


\subsubsection{Longitudinal tomography of the injected beam}

After verifying the credibility of the dipole scanning scheme, we carry out the measurement on the beamline. The dipole strength is scanned in the range where there is detectable beam signal at WGM. For each dipole strength, the WGM data is recorded and processed by the modulation-demodulation method. After combining all the results from the scanning, we have derived the beam energy spectrum as shown in Figure 4.25 and the longitudinal tomography as shown in Figure 4.26 and Figure 4.27 .

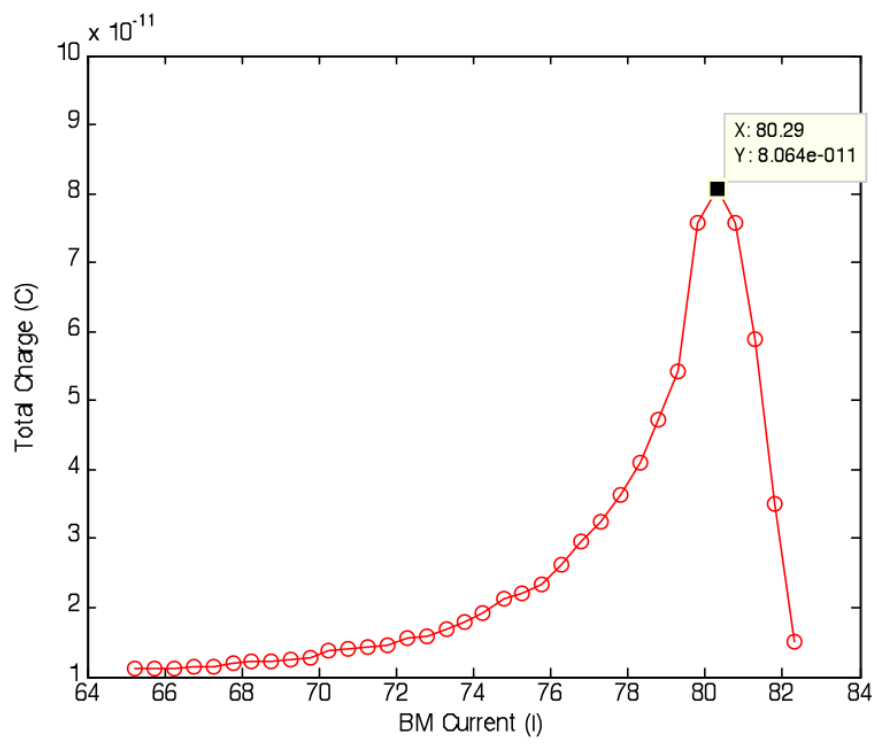

Figure 4.25: The energy spectrum of the LINAC beam. The dipole strength coresponds to the beam energy.

In the future, we will replace the current medical LINAC with a new S-band LINAC. This tomography scanning diagnosis scheme will be very useful to measure the new LINAC beam property and gives important information for the LINAC tuning. 


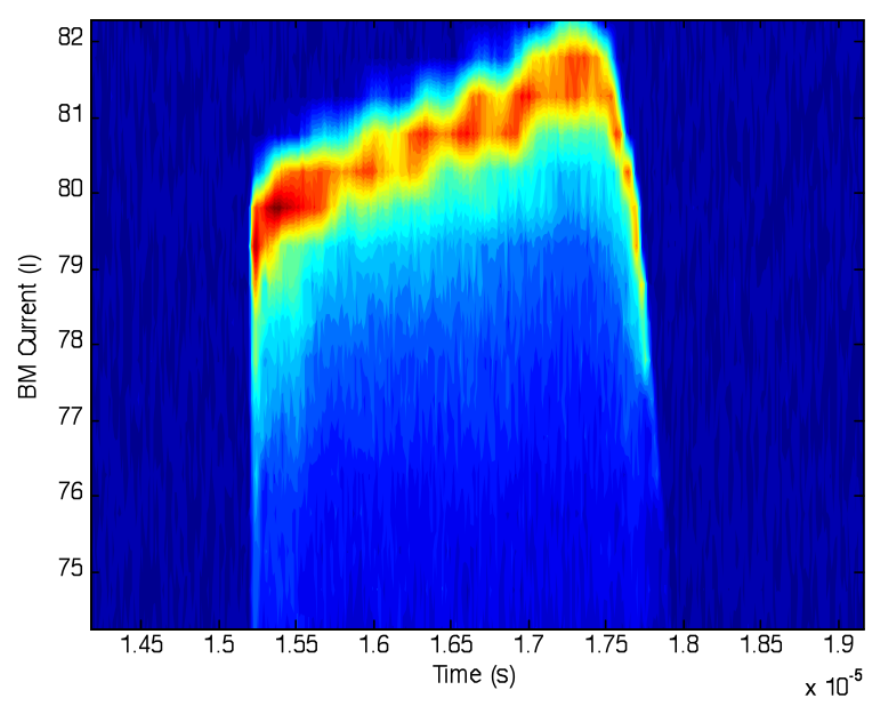

Figure 4.26: The 2D projection of the longitudinal phase space of the macro beam pulse.

\subsection{Extraction beam line measurement}

The ALPHA storage ring is designed to have a compaction factor of about 0.5. For the beam with a momentum spread of $\pm 0.5 \%$, the $2.856 \mathrm{GHz}$ micro bunches will be debunched in a signal pass.

To verify the debunching result, we move the WGM to the extraction line to measure the extracted beam. Due to the bandwidth limit of the WGM, we can't measure the length of the microbunch directly. Instead, we measure the $2.856 \mathrm{GHz}$ first harmonic strength in the FFT frequency spectrum, which will decrease if the debunching happens. If the beam is completely debunched into DC beam, the 2.856 $\mathrm{GHz}$ peak will disappear, as shown in Figure 4.19.

For the WGM data in the extraction line, we can no longer use the modulationdemodulation scheme, which is only valid when micro bunch length is significantly 


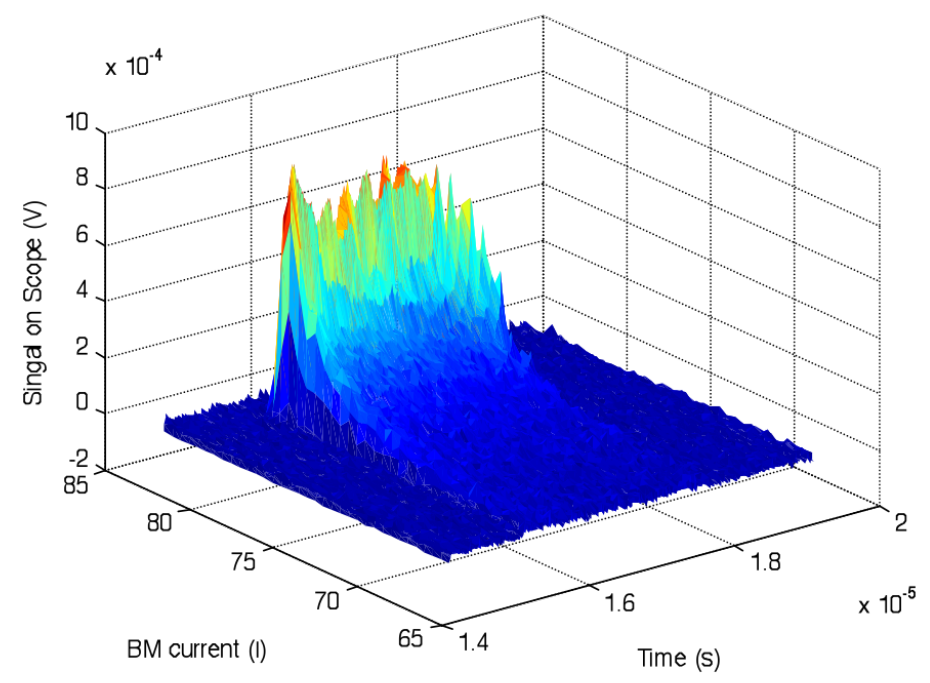

Figure 4.27: The 3D painting of the longitudinal phase space of the macro beam pulse.

shorter than the time interval between two adjacent beams. To avoid the low frequency cut from the amplifier, we use an amplifier with a low frequency limit of 50 $\mathrm{KHz}$. It is low enough for the $4 \mu \mathrm{s}$ macro pulse length and the DC part can be recovered by a baseline subtraction.

One problem is that the high frequency limit of this amplifier is only up to 500 $\mathrm{MHz}$. So the $2.856 \mathrm{GHz}$ strength has been reduced. However, this reduction is the same for both inject and extract beamline and we can take a ratio of the data to get rid of this problem. The DC component is easily recovered by a baseline subtraction. The comparison of injection and extraction beam frequency spectrum is shown in Figure 4.28.

For the injected beam,

$$
\mathrm{R}_{\text {inject }}=\frac{3.321 \times 10^{-4}}{7.744 \times 10^{-4}}=0.429
$$




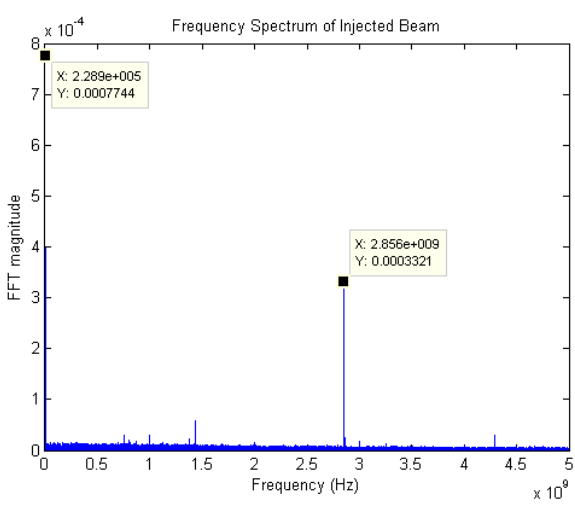

(a) Injected beam

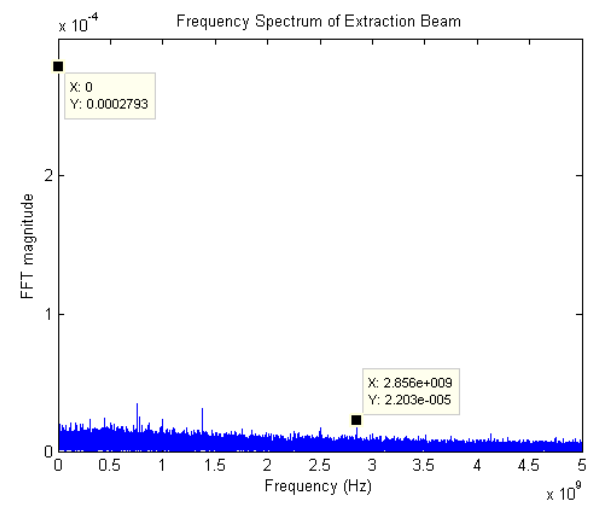

(b) Extracted beam

Figure 4.28: The frequency spectrum of the injected and extracted beam.

For the extracted beam,

$$
\mathrm{R}_{\text {extract }}=\frac{2.203 \times 10^{-5}}{2.793 \times 10^{-4}}=0.079
$$

So the normalized debunching factor $\mathrm{f}$ is:

$$
\mathrm{f}=\frac{\mathrm{R}_{\text {extract }}}{\mathrm{R}_{\text {inject }}}=0.184
$$

Thus we have verified the debunching effect of the ALPHA storage ring.

This extraction line measurement is done on a temporary extraction line and the aim is just to verify the debuching. Due to the time limit, the beam line is not optimized for the maximum transmission efficiency. Comparing the DC peak strength, we can calculate the transmission efficiency:

$$
T=\frac{2.793 \times 10^{-4}}{7.744 \times 10^{-4}}=36.7 \%
$$




\section{Chapter 5}

\section{Characterization of stored beam in}

\section{ALPHA ring}

In the accumulation mode of ALPHA operation, the injected beam can be accumulated and stored in the storage ring before being extracted by a fast kicker. In this chapter, we will evaluate the equilibrium beam emittance for the stored beam in ALPHA.

\subsection{Emittance of an electron storage ring}

Emittance is a property of a distribution of particles in phase space. Given a normalized distribution function $\rho\left(y, y^{\prime}\right)$, the moments of the beam distribution are:

$$
\begin{array}{r}
\langle y\rangle=\int y \rho\left(y, y^{\prime}\right) d y d y^{\prime} \quad\left\langle y^{\prime}\right\rangle=\int y^{\prime} \rho\left(y, y^{\prime}\right) d y d y^{\prime} \\
\sigma_{y}^{2}=\int(y-\langle y\rangle)^{2} \rho\left(y, y^{\prime}\right) d y d y^{\prime} \quad \sigma_{y^{\prime}}^{2}=\int\left(y^{\prime}-\left\langle y^{\prime}\right\rangle\right)^{2} \rho\left(y, y^{\prime}\right) d y d y^{\prime} \\
\sigma_{y y^{\prime}}=\int(y-\langle y\rangle)\left(y^{\prime}-\left\langle y^{\prime}\right\rangle\right) \rho\left(y, y^{\prime}\right) d y d y^{\prime}=r \sigma_{y} \sigma_{y^{\prime}}
\end{array}
$$


where $\sigma_{y}$ and $\sigma_{y^{\prime}}$ are the rms beam widths, $\sigma_{y y^{\prime}}$ is the correlation, and $\mathrm{r}$ is the correlation coefficient. The rms beam emittance is defined as

$$
\epsilon_{r m s}=\sqrt{\sigma_{y}^{2} \sigma_{y^{\prime}}^{2}-\sigma_{y y^{\prime}}^{2}}=\sigma_{y} \sigma_{y^{\prime}} \sqrt{1-r^{2}}
$$

The rms emittance is equal to the phase space area enclosed by the CourantSnyder ellipse of the rms particle. If the accelerator is composed of linear elements such as dipoles and quadrupoles, this emittance is invariant by the virtue of Poincare invariant [28].

For a low energy electron storage ring, important factors that affect the beam emittance are synchrotron radiation, quantum fluctuation, beam gas scattering, and Intra-beam Scattering (IBS). While the synchrotron radiation damps the beam emittance, the other three factors excite it to grow. When damping and growing effects cancel each other, the stored beam arrives at equilibrium state. As we will see later, these factors weight differently for beam operated under different conditions.

Both damping and excitation are dynamic processes in the storage ring. We use "growth/damping time" to characterize the time evolution of beam emittance. We assume a constant emittance growth/damping rate with:

$$
\alpha=-\frac{1}{\epsilon} \frac{d \epsilon}{d t}
$$

and find:

$$
\epsilon=\epsilon_{0} e^{-t / \tau}
$$

where the time constant $\tau=1 / \alpha$. The positive $\alpha$ means a damping process while the negative $\alpha$ is for a growing process. Each dynamic process has its own growth/damping time. The effective time constant $\tau_{\text {total }}$ is given by:

$$
\frac{1}{\tau_{\text {total }}}=\frac{1}{\tau_{1}}+\frac{1}{\tau_{2}}+\frac{1}{\tau_{3}}+\cdots
$$

Here we have a brief discussion of each factor and their corresponding time constants. 


\subsubsection{Synchrotron radiation}

The synchrotron radiation is the electromagnetic radiation generated by the acceleration of ultra relativistic charged particles. It is mainly concentrated in the forward direction along the beam trajectory. The energy loss due to synchrotron radiation is replenished by the RF cavity. This energy loss-regain process induces the emittance damping in all three dimensions.

The damping time in each dimension are:

$$
\begin{aligned}
\tau_{x} & =\frac{2 E T_{0}}{J_{x} U} \\
\tau_{z} & =\frac{2 E T_{0}}{J_{z} U} \\
\tau_{E} & =\frac{2 E T_{0}}{J_{E} U}
\end{aligned}
$$

$E$ is the beam nominal energy, $U$ is the total synchrotron radiation lost in one turn and $T_{0}$ is the revolution period. $J_{x}, J_{z}$ and $J_{E}$ are the damping partition number, which indicates the damping effect distribution in each dimention.

The emittance damping damping equations are:

$$
\begin{aligned}
\frac{d \epsilon_{x}}{d t} & =-\frac{2}{\tau_{x}} \epsilon_{x} \\
\frac{d \epsilon_{z}}{d t} & =-\frac{2}{\tau_{z}} \epsilon_{z} \\
\frac{d \delta}{d t} & =-\frac{1}{\tau_{E}} \delta
\end{aligned}
$$

\subsubsection{Quantum fluctuation}

Electromagnetic radiation is emitted in quanta of discrete energy. When a photon is emitted, the electron energy makes a small discontinuous jump. The emission time is short and thus the synchrotron radiation can be considered as instantaneous. Besides, the emission times of individual quanta are statistically independent. Since 
the energy of each photon is a very small fraction of the electron energy, the emission of successive quanta is a purely random process, which satisfies Poisson distribution.

Discontinuous quantized photon emission disturbs electron orbits. The cumulative effect of many such small disturbances introduces diffusion similar to random noise. The amplitude of oscillation will grow until it is balanced by the synchrotron radiation damping. In a similar way as the synchrotron radiation, we can characterize the quantum excitation by the emittance growth time :

$$
\begin{aligned}
\frac{d \epsilon_{x}}{d t} & =G_{x} \\
\frac{d \epsilon_{z}}{d t} & =G_{z} \\
\frac{d \delta}{d t} & =\frac{1}{4 \delta} G_{E}
\end{aligned}
$$

where:

$$
\begin{aligned}
G_{x} & =\frac{\left\langle N\left\langle\mu^{2}\right\rangle H\right\rangle}{E^{2}} \\
G_{z} & =\frac{\left\langle N\left\langle\mu^{2}\right\rangle\right\rangle\left\langle\beta_{z}\right\rangle}{\gamma^{2} E^{2}} \\
G_{E} & =\left\langle N\left\langle\mu^{2}\right\rangle H\right\rangle
\end{aligned}
$$

$N$ is the rate of photon emission and $\mu$ is the probable change in synchrotron motion amplitude. $\langle\cdots\rangle$ stands for the average of the ring.

\subsubsection{Gas scattering}

The gas scattering is the collision between the beam and the residual gas molecules. It includes both elastic and inelastic collision. In the elastic collision, the beam is deflected by gas nuclei resulting in an increase of the betatron oscillation amplitudes. In the inelastic collision, the electron loss energy either by bremsstrahlung radiation or direct energy transfer to residual gas molecule. 
The electron particle can be collided out of dynamic aperture or RF acceptance and be lost in a single collision. Or it can undergo a slow diffusion process due to multiple coulomb scattering (MCS). To study the emittance growth, we will focus only on the diffusion process. The life time due to the single collision loss will be treated later.

Assuming the gas pressure and temperature are identical everywhere along the storage ring vacuum chamber, in the transverse plane, the normalized emittance growth rate due to the MCS is [29]:

$$
\frac{1}{\epsilon_{n}} \frac{d \epsilon_{n}}{d t}=\frac{1}{2 \epsilon_{n}} \beta \gamma\langle\beta\rangle\left\langle\theta^{2}\right\rangle
$$

Thus for the physical emittance,

$$
\frac{d \epsilon}{d t}=\frac{1}{2}\langle\beta\rangle\left\langle\theta^{2}\right\rangle
$$

where $\langle\beta\rangle$ is the average beta function along the ring and $\left\langle\theta^{2}\right\rangle$ is the square of rms angle of MCS per time unit in the vacuum chamber. Summing over the species of average gas densities $n_{i}$,

$$
\left\langle\theta^{2}\right\rangle=\frac{4 \pi r_{e}^{2} c}{\beta^{3} \gamma^{2}} \sum_{i} n_{i} Z_{i}\left(Z_{i}+1\right) \ln \left(183 Z_{i}^{-1 / 3}\right)
$$

where $r_{e}$ is the classical radius of electron, and $Z_{i}$ is the atomic number of the gas species.

In the longitudinal direction, the lost energy due to the gas scattering is replenished by RF cavity. Similar to synchrotron radiation, this energy loss-replenish process will induce emittance damping. We can calculate this emittance damping rate in the same way as synchrotron radiation, except that the energy loss is now induced by gas scattering. 


\subsubsection{Intrabeam Scattering}

Intra-Beam Scattering (IBS) is a small angle multiple Coulomb scattering of charged particles in the beam. It causes small changes of the momenta of colliding particles and leads to a continuous increase of bunch dimensions and to a reduction of the beam lifetime when the particles hit the aperture. In proton or antiproton storage rings it causes, therefore, a fast decay of the luminosity, and in electron or positron storage ring with high particle densities for synchrotron light it reduces the brightness.

The IBS is different from the Touschek effect and the space charge effect, both of which are also caused by Coulomb force. The Touschek effect is a single Coulomb scattering effect where the energy transfer from horizontal to longitudinal direction leads to a large longitudinal momentum deviation. The intra-beam scattering, on the other side, is essentially a diffusion process in all three dimensions. The space charge effect is treated as a mean field of all the surrounding charged particles, ignoring the specific interaction between any two of them. It can be regarded as an average potential energy induced by all particles. The basic mechanism of IBS is the individual coulomb scattering process.

In the form of growth time, the emittance evolving equations due to the IBS effect can be written as:

$$
\begin{aligned}
\frac{d \epsilon_{x}}{d t} & =\frac{1}{\tau_{x}} \epsilon_{x} \\
\frac{d \epsilon_{z}}{d t} & =\frac{1}{\tau_{z}} \epsilon_{z} \\
\frac{d \delta}{d t} & =\frac{1}{2 \tau_{E}} \delta
\end{aligned}
$$

Without coupling and strong focusing $\left(\beta^{\prime}, D^{\prime} \approx 0\right)$, the IBS growth times in three dimensions are given by [30]. They are all proportional to a constant $A$ :

$$
A=\frac{r_{p}^{2} c_{l} N_{b}}{64 \pi^{2} \sigma_{s} \sigma_{p} \epsilon_{x b} \epsilon_{z b} \beta^{3} \gamma^{4}}
$$


where $N_{b}$ is the number of electron per bunch, $r_{p}$ is the classical particle radius, $\epsilon_{x b}$ and $\epsilon_{z b}$ are the horizontal and vertical emittance, $\sigma_{s}$ and $\sigma_{p}$ is the RMS of the bunch length and momentum spread, $\beta$ and $\gamma$ are relativistic factors and $c_{l}$ is the speed of light.

Even from this constant $A$, we can see that IBS is important for the low energy, high current beam. As the energy goes up or beam current goes down, the IBS effect will decrease.

More comprehensive IBS study (e.g., [31] and [32]) has also been carried out and the calculation for the growth time is more complicated. A number of codes have been developed for the IBS calculation and integrated into the popular beam dynamics calculation program, like ZAP [33] and MADX [34] and ELEGANT [35]. We will use MADX to calculate the IBS effect for the ALPHA storage ring.

\subsection{Equilibrium Emittance Calculation of ALPHA Storage Ring}

The major lattice parameters of the ring under accumulation mode, as well as other constants used in the calculation, are shown in Table 5.1. The twiss parameters along the ring are shown in Figure 5.1 and Figure 5.2.

Table 5.1: ALPAH Storage Ring Parameters

\begin{tabular}{|l|c||l|c||l|c|}
\hline Energy E & $50 \mathrm{MeV}$ & Circumference C & $20 \mathrm{~m}$ & $\rho_{\text {dip }}$ & $1.273 \mathrm{~m}$ \\
\hline$\rho_{\text {wig }}$ & $0.75 \mathrm{~m}$ & $C_{\gamma}$ & $8.846 \times 10^{-14} \mathrm{~m} /(\mathrm{MeV})^{3}$ & $L_{\text {wig }}$ & $0.4 \mathrm{~m}$ \\
\hline$J_{x}$ & 0.91148 & $J_{z}$ & 1 & $J_{E}$ & 2.0885 \\
\hline
\end{tabular}




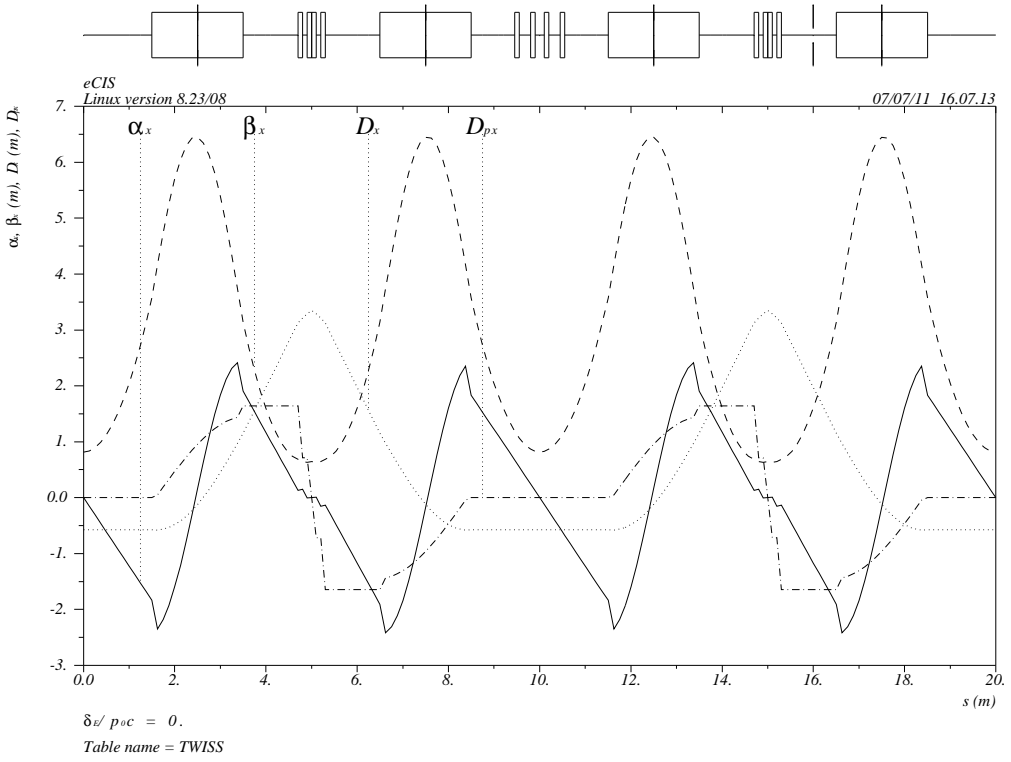

Figure 5.1: Horizontal twiss parameters along the ring. $\alpha_{x}$ and $\beta_{x}$ are the Courant-Snyder parameters; $D_{x}$ and $D_{p x}$ are the dispersion function and its derivative. 


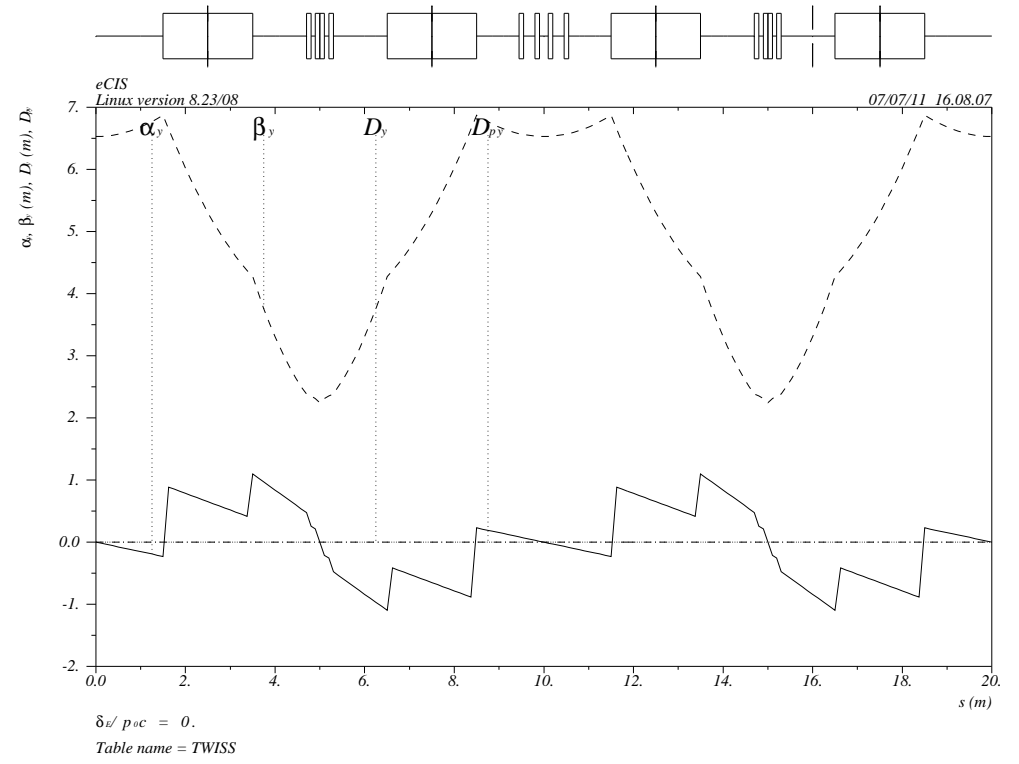

Figure 5.2: Vertical twiss parameters along the ring. $\alpha_{y}$ and $\beta_{y}$ are the Courant-Snyder parameters; $D_{y}$ and $D_{p y}$ are the dispersion function and its derivative. 
Applying the above equations, we can calculate the damping/growth time of each factor for the ALPHA storage ring and estimate the equilibrium emittance for the storage mode. To keep the calculation consistent, all the time factors are for the normalized emittance.

\subsubsection{Synchrotron radiation}

The synchrotron radiation happens in both bending dipole and damping wiggler. The total lost energy is:

$$
U_{\text {total }}=C_{\gamma} E^{4}\left(\frac{1}{\rho_{\text {dip }}}+\frac{L_{w i g}}{2 \pi \rho_{w i g}^{2}}\right)
$$

The damping partition numbers $J_{x}, J_{z}$ and $J_{E}$ are calculated by MAD8 and the values are shown in Table 5.1. After plugging in all the parameters, the normalized emittance damping equations due to synchrotron radiation are:

$$
\begin{aligned}
& \frac{d \epsilon_{x}}{d t}=-1.086 \times 10^{-6} E^{3} \epsilon_{x} \\
& \frac{d \epsilon_{z}}{d t}=-1.144 \times 10^{-6} E^{3} \epsilon_{z} \\
& \frac{d \delta}{d t}=-1.258 \times 10^{-6} E^{3} \delta
\end{aligned}
$$

\subsubsection{Quantum fluctuation}

Considering the synchrotron radiation in both dipole and damping wiggler, the quantum fluctuation in three dimensions are:

$$
\begin{aligned}
G_{x} & =\frac{1}{E^{2} C} C_{\mu} C_{\gamma} \frac{3}{4 \pi} \frac{\hbar c^{2}}{\left(m c^{2}\right)^{3}} E^{7}\left(\frac{2 \pi \rho_{\text {dip }}}{\rho_{\text {dip }}^{3}}\langle H\rangle_{\text {dip }}+\frac{L_{w i g}}{\rho_{\text {wig }}^{3}}\langle H\rangle_{w i g}\right) \\
G_{z} & =G_{E} \frac{\left(m c^{2}\right)^{3}}{E^{4}}\left\langle\beta_{z}\right\rangle_{\text {ring }} \\
G_{E} & =\frac{1}{C} C_{\mu} C_{\gamma} \frac{3}{4 \pi} \frac{\hbar c^{2}}{\left(m c^{2}\right)^{3}} E^{7}\left(\frac{2 \pi}{\rho_{\text {dip }}^{2}}+\frac{L_{w i g}}{\rho_{\text {wig }}^{3}}\right)
\end{aligned}
$$


After plugging in all the parameters, the normalized emittance growing equations due to the quantum fluctuation are:

$$
\begin{aligned}
& \frac{d \epsilon_{x}}{d t}=2.7 \times 10^{-17} E^{5} \\
& \frac{d \epsilon_{z}}{d t}=4.1 \times 10^{-18} E^{3} \\
& \frac{d \delta}{d t}=7.53 \times 10^{-19} E^{5} / \delta
\end{aligned}
$$

\subsubsection{Gas scattering}

A Residual Gas Analyzer (RGA) result shows that the $99 \%$ of the gas in the storage ring is $\mathrm{H}_{2}$ and the rest are traces of $\mathrm{CO}, \mathrm{CO}_{2}, \mathrm{H}_{2} \mathrm{O}$, Argon, He, etc. Without losing accuracy, we can assume all the gas is $\mathrm{H}_{2}$. The temperature is set at $300 \mathrm{~K}$.

In the transverse direction, the normalized emittance growth rates are calculated as:

$$
\begin{aligned}
& \frac{d \epsilon_{x}}{d t}=1.5 \times 10^{-5} P / E^{2} \\
& \frac{d \epsilon_{z}}{d t}=2.2 \times 10^{-5} P / E^{2}
\end{aligned}
$$

In the longitudinal direction, after the calculation, we find that the energy loss due to gas scattering is negligible compared with the synchrotron radiation. Thus we can ignore the damping effect.

\subsubsection{Intrabeam Scattering}

The growth times of Intrabeam Scattering $\tau_{i b s}$ are calculated by MADX.

\subsubsection{Transverse Linear Coupling}

Due to any magnet skew components and misalignments, there is inevitable linear coupling between horizontal and vertical emittance in the transverse plane. For the 
vertical emittance, which has been strongly damped by the synchrotron radiation, the linear coupling will induce a lot of emittance growth. To describe the impact of linear coupling on the vertical emittance, we introduce the term:

$$
\frac{d \epsilon_{z}}{d t}=1.144 \times 10^{-6} E^{3} \epsilon_{x} \times \kappa
$$

where $\kappa$ is the linear coupling constant, varying from 0 to 1 , corresponding from no linear coupling to complete coupling. This term makes sure that when energy is high and both gas scattering and IBS can be neglected, the transverse emittances at equilibrium state have the relation:

$$
\epsilon_{z}=\epsilon_{x} \times \kappa
$$

\subsubsection{The calculation procedure}

Combining each term together, we can write the time evolution equation of the emittances $\epsilon_{x(z)}$ and the momentum spread $\delta$ as:

$$
\begin{aligned}
& \frac{d \epsilon_{x}}{d t}=-1.086 \times 10^{-6} E^{3} \epsilon_{x}+2.7 \times 10^{-17} E^{5}+1.5 \times 10^{-5} P / E^{2}+\frac{1}{\tau_{x i b s}} \epsilon_{x} \\
& \frac{d \epsilon_{z}}{d t}=-1.144 \times 10^{-6} E^{3} \epsilon_{z}+1.144 \times 10^{-6} E^{3} \epsilon_{x} \times \kappa+4.2 \times 10^{-18} E^{3} \\
& \quad+2.2 \times 10^{-5} P / E^{2}+\frac{1}{\tau_{z i b s}} \epsilon_{z} \\
& \frac{d \delta}{d t}=-1.258 \times 10^{-6} E^{3} \delta+7.53 \times 10^{-19} E^{5} / \delta+\frac{1}{2 \tau_{\text {Eibs }}} \delta
\end{aligned}
$$

The calculation procedure is:

1. Set initial emittance $\epsilon_{x 0}, \epsilon_{z 0}$ and $\delta_{0}$.

2. For given energy, peak current, pressure and coupling constant, use MADX to calculate the IBS growth times $\tau_{x i b s}, \tau_{z i b s}$ and $\tau_{\text {Eibs }}$. 
3. From the evolution equation above, calculate the emittance changing rate $d \epsilon_{x(z)} / d t$ and $d \delta / d t$.

4. Choose the proper time step $\Delta T \ll \operatorname{Min}\left(\tau_{x i b s}, \tau_{z i b s}, \tau_{\text {Eibs }}\right)$. With the changing rates from step 3 , calculate the new emittances.

5. Go back to step 2 and repeat the iteration until the emittance growth rates are 0 , when emittances reach equilibrium.

One advantage of this tracking scheme is that the final equilibrium emittances are independent of the initial condition. Another is that it can give an estimation of the relaxing time of this dynamic process.

\subsection{Estimation Results and discussion}

With the calculation scheme described above, we calculated the equilibrium emittance under different conditions.

Figure 5.3 shows how the equilibrium horizontal emittance change with beam energy. The beam current is set at $25 \mathrm{~A}$, which is the requirement of the storage beam. The results under different pressures from 1 nTorr to 0.01 nTorr are almost the same, which means the gas scattering effect is not important here. For the low energy beam, due to the IBS, the emittance has been severely blown up. As the energy increases, the IBS effect decreases and the emittance is dominated by the balance between synchrotron radiation and quantum fluctuation. This emittance is called the natural emittance and is proportional to the square of energy. Thus we see the emittance starts to grow after around $200 \mathrm{MeV}$.

Figure 5.4 and Figure 5.5 show how the equilibrium horizontal and vertical emittance change with beam current. The beam energy is set at $50 \mathrm{MeV}$, which is the designed energy of the extracted beam. At $50 \mathrm{MeV}$, IBS is a dominant factor on the 


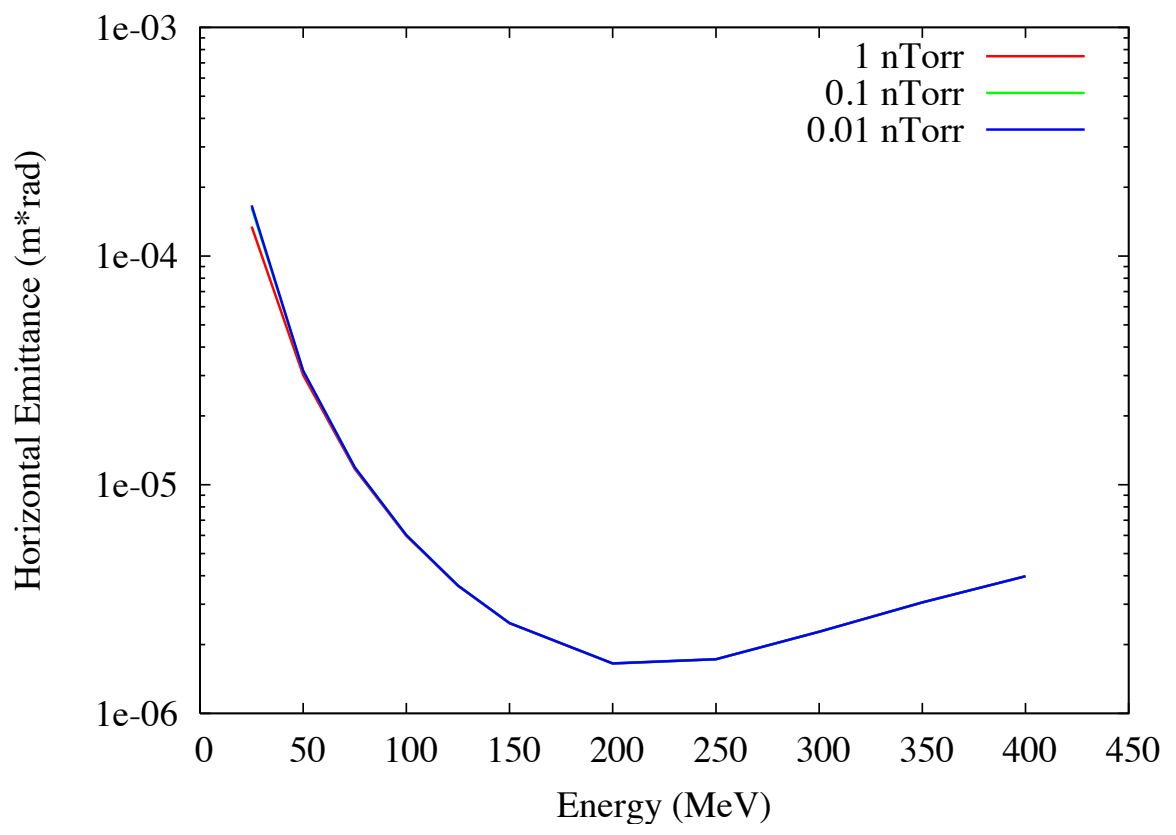

Figure 5.3: Horizontal emittance with different beam energies, with beam current $25 \mathrm{~A}$ 


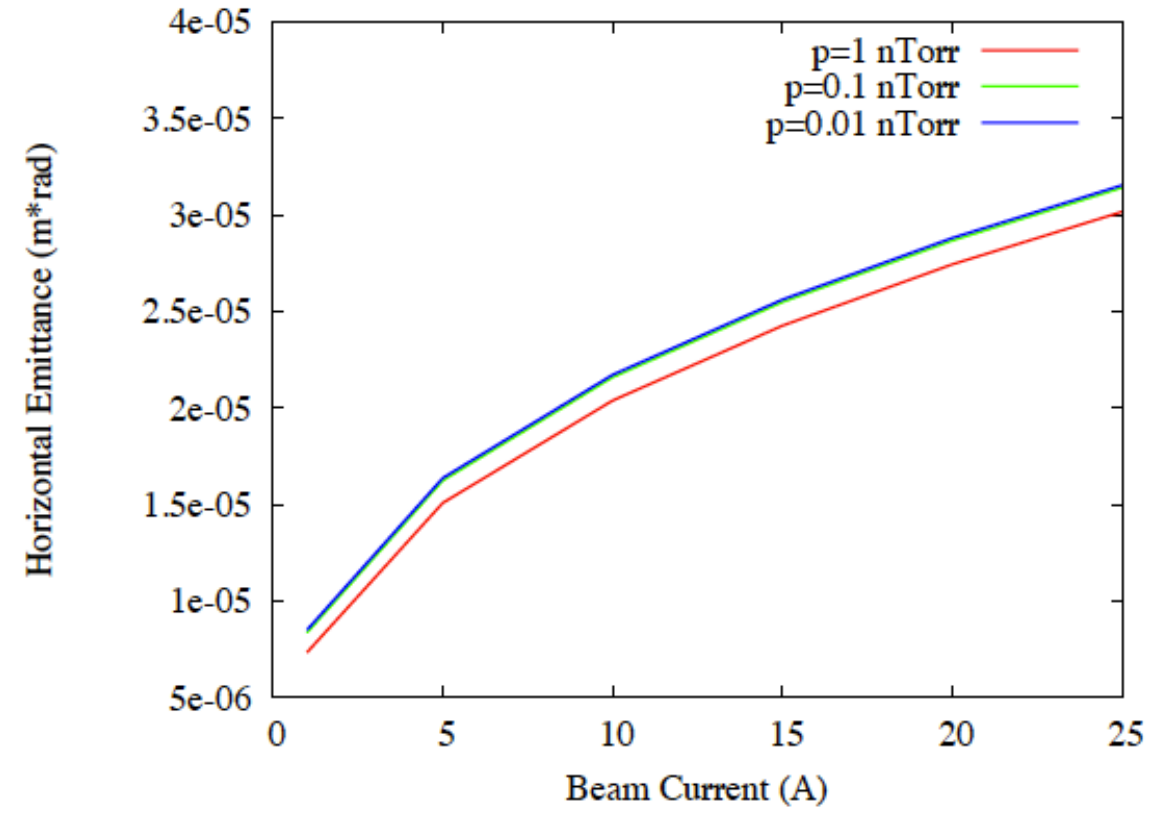

Figure 5.4: Horizontal emittance with different beam currents, with beam energy $50 \mathrm{MeV}$ 


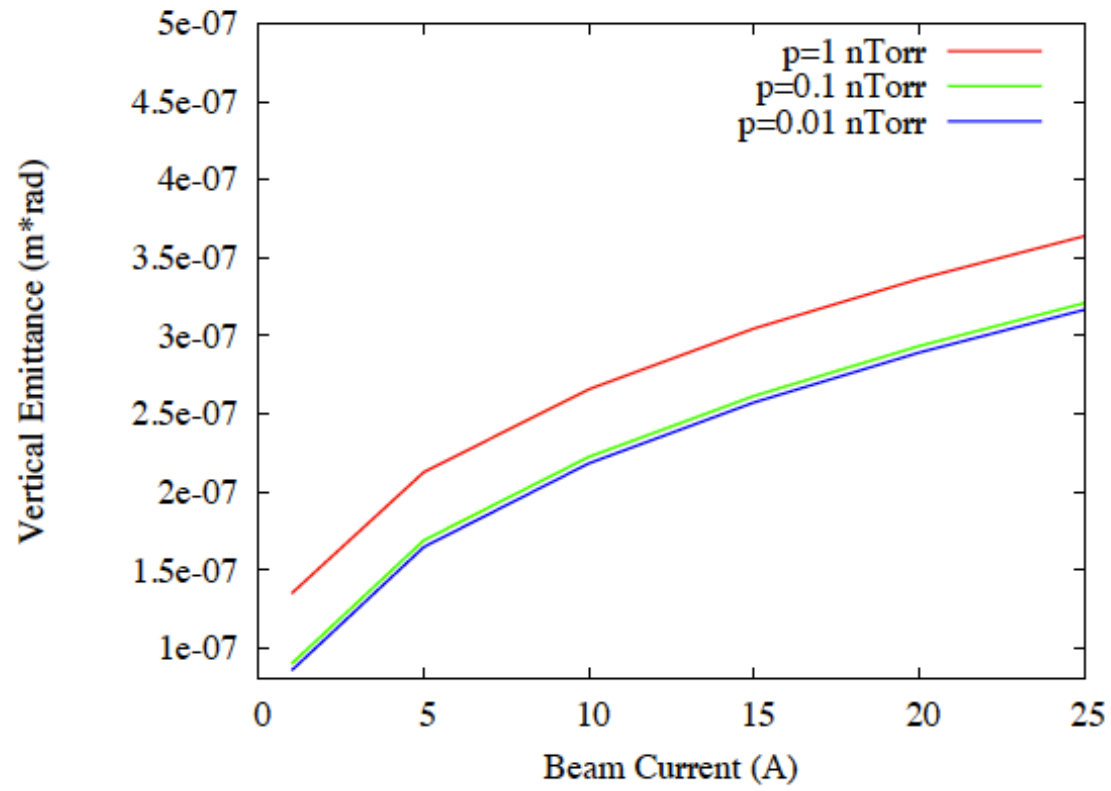

Figure 5.5: Vertical emittance with different beam currents, with beam energy $50 \mathrm{MeV}$ 
beam emittance.As the beam current increases, the IBS effect increases and the beam emittance grows. At $50 \mathrm{MeV}$, IBS is a dominant factor on the beam emittance. At $25 \mathrm{~A}$, the horizontal emittance $\epsilon_{x}$ is about $30 \mu \mathrm{m}$. The beam RMS size

$$
\sigma_{x}=\sqrt{\epsilon \beta_{x}}
$$

With beta function $\beta_{x}$ varies from $0.6 \mathrm{~m}$ to $6.5 \mathrm{~m}$ in the ring, the beam RMS size varies from $4.2 \mathrm{~mm}$ to $14.0 \mathrm{~mm}$.

The vertical emittance is much smaller than the horizontal emittance due to the relativistic effect in the synchrotron radiation damping. To reduce the horizontal emittance, we can do the linear coupling between horizontal and vertical emittance, by using skew quadrupole or solenoid. Figure 5.6 shows how the horizontal and vertical emittance change when changing the linear coupling constant. $\kappa$. 


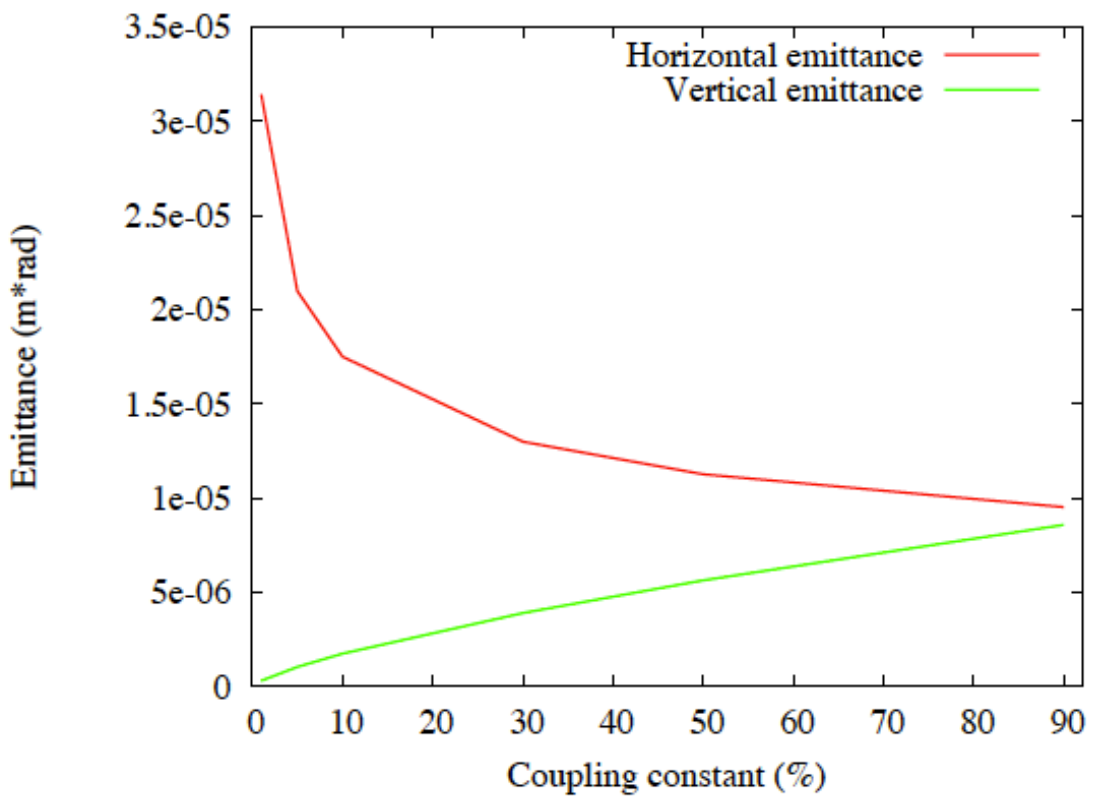

Figure 5.6: Horizontal and vertical emittance with different coupling constant $\kappa$, with beam energy $50 \mathrm{MeV}$ and beam current 25 A. 


\section{Chapter 6}

\section{Conclusion}

The instrumentation work, initial beam measurements and beam dynamics study on the Advanced eLectron-PHoton fAcility (ALPHA) has been described in this dissertation.

To overcome the Touschek effect and provide enough beam life time, an extensive study has been carried out to build a first harmonic cavity for ALPHA. Both transmission line model and SUPERFISH simulation are applied to analyze the cavity. A used ferrite loaded cavity from Cooler Injector Synchrotron (CIS) has been refurbished. The cavity size is reduced to fit into the tight space in ALPHA, also the shunt impedance $R_{s}$ is improved from $300 \Omega$ to $1200 \Omega$ in low power measurement. The quality factor $Q$ is measured to be 13 , mainly determined by the quality factor of ferrite material. In high power measurement, $R_{s}$ has achieved $700 \Omega$ at $300 \mathrm{~W}$ input power. However, due to the High Loss Effect (HLE) limitation, the refurbished cavity might not be able to provide enough voltage for ALPHA operation. Thus we have designed a new cavity with similar structure but a different and proper ferrite material. The new cavity is designed to be more compact, more efficient and operated below the threshold of HLE. By the time of this thesis, a new ferrite has been built, tested 
and installed in the ring. The low level RF control system is under development.

A stripline traveling wave kicker has been designed for the fast extraction. Characteristic impedance of the kicker is designed to be $50 \Omega$ to minimize the time response of the kicking field and maximize the power transmission efficiency. The simulation in Microwave Studio shows a rise time less than 3 ns and a power reflection less than $-20 \mathrm{~dB}$. The kicker voltage requirement depends on the strip length and kicking angle. For a $30 \mathrm{~cm}$ strip and $12 \mathrm{~mm}$-mrad kicking angle, the required voltage is $+/-25 \mathrm{kV}$ on each electrode. By the time of this thesis, several engineering challenges have been solved and the kicker is ready for fabrication.

With a wall gap monitor (WGM), the longitudinal beam characterization has been carried out in the single pass commissioning. Before installing it in the ring, we have measured the effective impedance $R$ and frequency bandwidth of the WGM on the bench test. Due to the small beam current and large Klystron noise, proper shielding and amplification are needed to get a good beam signal. With WGM in the injection line, we have measured the current, the charge and the longitudinal profile of the injected beam. A modulation-demodulation data processing scheme is developed to suppress the low frequency noise and recover the DC information which is cut off by the amplifier. By scanning the 45 degree dipole strength in the injection line, we have reconstructed the tomography of longitudinal phase space of LINAC beam. This diagnosis will be very useful for the commissioning of the upgraded LINAC later. With WGM in the extraction line, we have measured the extracted beam signal spectrum. Comparing with the injection line, we have observed the significant suppression of the $2.856 \mathrm{GHz}$ spectrum line, and thus verified the debunching effect of ALPHA storage ring.

In the accumulation mode, ALPHA will store up to $25 \mathrm{~A}$ beam at $50 \mathrm{MeV}$. For such a high current, low energy electron beam, the intra beam scattering (IBS) is a significant factor to determine the beam size. Including the effect of IBS and gas 
scattering, a self consistent simulation has been carried out to estimate the emittance of the stored beam under different operating conditions. The simulation also shows the effect of linear coupling to reduce the beam size.

By the time of this thesis, ALPHA has successfully complished the single pass commissioning and demonstrated both debunching effect and non-linear beam spreading. The preparation for the accumulation mode is under way and the commissioning will start soon.

With the successful construction of ALPHA, both radiation effect testing and beam physics study will be carried out. The debunching function of the ALPHA storage ring can reduce the RF noise from LINAC beam in electronics testing. The non-linear beam spreading scheme improves both uniformity and efficiency of the radiation at the test station. On beam physics, ALPHA is a ideal platform to study the IBS effect, the Touschek effect and the performance of the Gradient Damping Wiggler. 


\section{Bibliography}

[1] E. Rutherford Philos. Mag. 21 669, 1911.

[2] O. S. Bruning et al., "Lhc design report. 1. the lhc main ring," CERN-2004-003.

[3] R. L. Kustom, "An Overview of the Spallation Neutron Source project," LINAC2000-TU101, physics/0008212.

[4] J. N. Corlett et al., "Design Studies for a VUV-Soft X-ray FEL Facility at LBNL," PAC WEPEA067, 2010.

[5] D. Neuffer AIP Conf. Proc. 156 201, 1987.

[6] C. M. Ankenbrandt et al. Phys. Rev. ST Accel. Beams 2 (1999) 081001.

[7] M. M. Alshoro'a et al. Phys. Rev. ST Accel. Beams 6 (2003) 081001.

[8] R. B. Palmer et al. PAC07-THPMS090, arXiv:0711.4275.

[9] e. J. Doskow, "The ALPHA project at IU CEEM," IPAC-2010-MOPEA082.

[10] S.Y. Lee et al, "Low energy electron storage ring with tunable compaction factor," Review of Science Instruments 78, 075107, 2007.

[11] A. Piwinski, "The touschek effect in strong focusing storage ring," DESY 98-179. 
[12] X.Kang, The Cooler Injector Synchrotron in IUCF. PhD thesis, Indiana University Bloomington, 1998.

[13] D. McGinnis, "Introduction to rf for particle accelerators," tech. rep., USPAS, 2007.

[14] S. Tantawi, "Lecture notes of microwave instrumentation and measurements," tech. rep., USPAS, 2009.

[15] J. Byrd, "Rf cavity bead pull measurements," Lecture notes of class Microwave Measurements Laboratory, USPAS and CCAST Beijing, China, 1998.

[16] K. Kaspar et al., "Studies on maximum rf voltages in ferrite-tuned accelerating cavities," EPAC, 2004.

[17] J. E. Griffin and G. Nicholls, "Notes on high loss effect in rf cavity tuning ferrite," Tech. Rep. TM-655,0334.050, Fermilab, May 1976.

[18] C. Beltran, Study of the longitudinal space charge compensation and longitudinal instability of the ferrite inductive inserts in the Los Alamos proton storage ring. $\mathrm{PhD}$ thesis, Indiana University Bloomington, 2003.

[19] Gary East, "Specification for the electrostatic kicker system," tech. rep., IUCEEM, 2008.

[20] http://laacg1.lanl.gov/laacg.

[21] www.cst.com.

[22] S. De Santis et al, "Fast extraction kicker for the accelerator test facility," PAC, 2007. 
[23] David Alesini et al, "Design, test and operation of new tapered stripline injection kickers for the $e^{+} e^{-}$collider da $\phi$ ne," PRSTAB 13, 111002, 2010.

[24] R. C. Webber, "Tutorial on beam current monitoring," Fermilab-Conf-00-119, 2000.

[25] P. Forck, "Lecture notes on beam instrumentation and diagnositcs," Joint University Accelerator School.

[26] Robert C. Webber, "Longitudinal emittance: An introduction to the concept and survey of measurement techniques including design of a wall current monitor," AIP Conf. Proc, Volume 212, pp. 85-126, 1990.

[27] http://www $\cdot$ muonsinc $\cdot$ com/tiki-index $\cdot$ php?page=G4beamline.

[28] S. Lee, Accelerator Physics. World Scientific, 2004.

[29] N. V. Mokhov and V. I. Balbekov, "Beam and luminosity lifetime," 1998.

[30] A. Piwinski, "Intrabeam scattering," Proc. Ninth Int. Conference on high Energy Accelerators, 1975.

[31] J.D. Bjorken, S. K. Mtingwa, "Intrabeam scattering," Part. Acc. Vol. 13, 1983.

[32] K. Kubo, K. Oide, "Intrabeam scattering in electron storage rings," PRSTAB 4, 124401, 2001.

[33] J. B. M.Zisman, S. Chattopadhyay, "Zap user's manual," tech. rep., LBL-21270, 1986.

[34] "The mad-x program." http://mad.web.cern.ch/mad/. 
[35] "User's manual for elegant." http://www.aps.anl.gov/Accelerator_Systems Division/Accelerator_Operations_Physics/manuals/elegant_latest/elegant .html . 


\title{
Tianhuan Luo
}

\author{
Indiana University Center for Exploration of Energy and Beam \\ 2401 N Milo B. Sampson Lane Bloomington, IN 47408 \\ Tel: 812-855-5190(Office) \\ Email: luo@indiana.edu
}

\section{$\underline{\text { Education }}$}

Degree Major Institution

Ph.D. (08/2011) Accelerator Physics Indiana University, Bloomington

M. S. (12/2007) Physics Indiana University, Bloomington

B. S. (06/2006) Applied Physics $\quad$ USTC, Hefei, P.R.China

\section{Fellowships and Awards}

- Undergraduate Student Fellowship, USTC, Dec.2002 - June 2005

\section{Professional Experience}

- Indiana University Advanced eLectron-PHoton Facility (Research Associate, $01 / 2010$ - 08/2011)

- Indiana University Physics Department Accelerator Physics Group (Research Associate, 10/2008 - 08/2011)

- United States Particle Accelerator School, San Francisco (Associate Instructor, 01/2010)

- Indiana University, Bloomington (Associate Instructor, 08/2006-12/2008) 